\title{
Bioaccessibility and bioavailability of phenolic compounds
}

\author{
Fereidoon Shahidi* and Han Peng
}

Department of Biochemistry, Memorial University of Newfoundland, St. John's NL, Canada A1B 3X9

*Corresponding author: Fereidoon Shahidi, Department of Biochemistry, Memorial University of Newfoundland, St. John's NL, Canada A1B 3X9. Tel: 709864 8552; Fax: 709864 2422; E-mail: fshahidi@mun.ca

DOI: $10.31665 / J F B .2018 .4162$

Received: October 8, 2018; Revised received \& accepted: November 7, 2018

Citation: Shahidi, F., and Peng, H. (2018). Bioaccessibility and bioavailability of phenolic compounds. J. Food Bioact. 4: 11-68.

\begin{abstract}
Modern epidemiological and interventional studies have demonstrated that various bioactivities including antioxidant, antiproliferative, immune-regulatory, hormonal-regulation abilities and neuro-/hepato-/cardioprotective effects result from consumption of a phenolic-rich diet. The health benefits of ingesting phenolics are greatly dependent on their bioaccessibility and bioavailability in the digestive tract and circulatory system. This contribution attempts to review the bioaccessibility and bioavailability of phenolic compounds by focusing on the body's internal mechanism including digestion, absorption, transport, modification, excretion, and colonic fermentation. The bioaccessibility and bioavailability of different phenolics vary depending on the physical condition of an individual, including digestive/absorptive/metabolic/response capability and effective dose. External factors such as processing methods and interaction with various food matrices also play a vital role on the bioavailability of dietary phenolic compounds. On the other hand, some novel phenolics have been synthesized to enable them rendering new bioactivities. The key internal factors influencing the bioaccessibility and bioavailability are also reviewed in this contribution. In addition, suggestions have been made for future measurement and assessment of bioavailability, together with prospects for food/nutraceutical/pharmaceutical application of novel phenolics.
\end{abstract}

Keywords: Phenolics; Bioavailability; Transporters; In vivo metabolism; Colonic catabolism.

\section{Introduction}

Dietary phenolic compounds constitute one of the most important groups of natural antioxidants and chemopreventive agents. They include phenolic acids, flavonoids, stilbenes, coumarins, lignans, lignins, and oligomeric and polymeric proanthocyanidins, among others. Numerous epidemiological and interventional studies have demonstrated that consumption of phenolic-rich foods is inversely associated with the risk of most common oxidative stress-associated degenerative and chronic diseases, including cardiovascular disease (CVD), type-II diabetes mellitus, cancer and aging (Amarowicz and Pegg, 2008; Villegas et al., 2008). A balanced diet provides many different phenolic compounds and seasonal and cultural dietary changes in every country result in their differential bioavailability. In addition, the bioaccessibility and bioavailability of different phenolics vary depending on individuals' physical condition, including digestive/absorptive or metabolic ability and effective dose. Besides common phenolics that serve as antioxidants, some novel phenolic compounds have been discovered, synthesized or modified to reveal new properties as well as enhancing their original bioactivities such as antioxidant, antimicrobial, antiproliferative, immune-regulatory, hormonal-regulation abilities and neuro-/hepato-/cardioprotective effects (Maeda-Yamamoto et al., 2017; Patel, 2014; Peng et al., 2017). However, excessive exposure to phenolics upon dietary ingestion and intravenous injection may also render adverse effects on health (Galati and O'brien, 2004; Skibola and Smith, 2000; Watjen et al., 2005). In comparison, modified lipophilic phenolics, as well as the production of small size nanoparticles, may exert toxicity at a lower dose while few studies have so far been conducted to support such a hypothesis. Therefore, in considering phenolic compounds, it is imperative to remember both their nutraceutical potential and application limitation; it is also important to know the content of phenolics present in specific food or dietary supplement and their bioavail- 


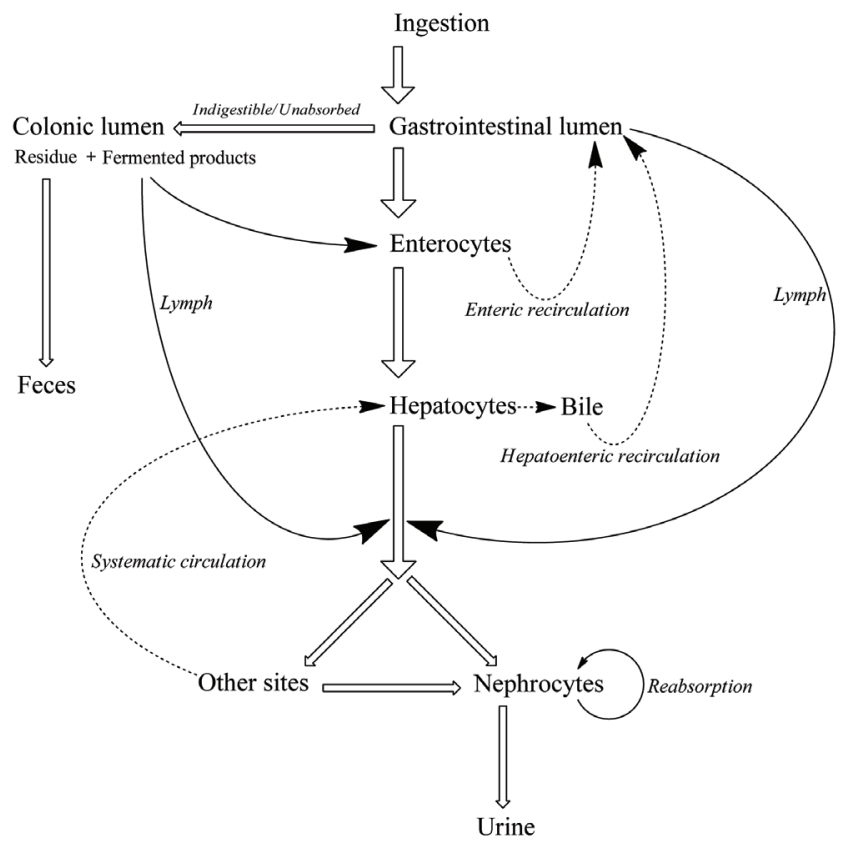

Figure 1. Schematic representation of phenolic bioavailability.

ability.

The health benefits of ingested phenolics greatly depend on their bioaccessibility and bioavailability in the digestive tract and circulatory system. Among different phenolics, bioavailability differs greatly, the ones with most abundance or highest in vitro activity in the daily diet do not necessarily positively correlate to those with the best bioaccessibility and bioavailability profile (CarbonellCapella et al., 2014). Several decisive steps, including the effects of digestion rate, first passing effect, metabolic modification and colonic fermentation have attracted much attention in order to investigate the generalized evaluation method of bioavailability for phenolics-dominated functional foods, nutraceuticals, dietary supplements, and drugs. The structural characteristics including functional groups and polymerization enable phenolics to present different solubilities and be absorbed and metabolized in their own pathway, therefore also resulting in a varied function of phenolics, compared to other phytochemicals. Meanwhile, external factors play a vital role in bioaccessibility and bioavailability of phenolics upon ingestion. Both interaction with various food matrix components and diverse processing methods significantly influence the actual bioaccessibility and bioavailability of ingested phenolics. This contribution summarizes digestion, absorption, metabolism, circulation and excretion process, as well as biological function and potential application of natural and synthetic phenolics.

\subsection{Definition of bioaccessibility and bioavailability}

The chemical and physiological effects of natural bioactive components in food manufacturing and dietary evaluation, and even in phytopharmacological studies are well recognized. Among functional ingredients, the effects of phenolics on human health are undeniable, not only as nutraceuticals and additives but also as therapeutic agents (Ferreira et al., 2017). Most of the action mechanisms and related pharmacokinetic parameters that depend on phenolics uptake are still poorly demonstrated and clarified. Meanwhile, based on in vivo observational health effects, many studies focusing on bioactivity assessment and in vitro cell pathway investigations are still in progress. The results so obtained are cell line-dependent. However, bioactivities of phenolics are compared and reported often not under similar in vitro conditions in different reports. Besides, bioactivity experiments conducted in vitro especially in cell line tests are also concentration-dependent, and the actual phenolic metabolites are not what they originally were, hence consideration of realistic concentrations and metabolites in action sites which is determined by bioaccessibility and bioavailability criteria is essential.

On the other hand, different from common nutritional compounds, phenolics are also regarded as anti-nutrients and nonnutritive bioactive compounds. Normally, all foods we eat are composed of nutrients and/or non-nutrients. Food nutrients are mainly carbohydrates, proteins, lipids, vitamins, minerals, and water and serve as a source for energy production, tissue/organ construction, and co-factors in intermediary metabolisms, while non-nutrients could be indigestible polysaccharides, phytochemicals, medical ingredients, and several inactive substances that may be in charge of mechanism modulation, disease prevention/treatment, and various other biological functions (Kanazawa, 2011; Pan et al., 2018; Velderrain-Rodríguez et al., 2014). For both nutrients or non-nutrients, bioaccessibility and bioavailability could be regarded as bio-efficiency. Specifically, bioaccessibility is the digestion and absorption efficiency (or digestibility and absorptivity) of a certain food constituent or drug ingested by oral administration, normally expressed as a percentage of the actual amount released and absorbed constituent to its total content. However, for bioavailability, there are significant differences between that of nutrients and non-nutriments. In the nutrition area, bioavailability is crudely defined as the utilized or stored proportion of the total administered quantity. For non-nutrients, such as medicines, bioavailability is more strictly defined. According to 21 CRF 314.3 (2016) of Food and Drug Administration (FDA), Code of Federal Regulations, bioavailability of drugs is defined as the available ratio of active ingredient or active moiety absorbed and detected in the target site to the total amount of orally ingested drug products; the intravenously administrated medication is defined as having a bioavailability of $100 \%$.

Bioactive compounds or bioactives refer to substances that have an effect on human health; this kind of compounds are distinguished from both nutrients and non-nutrients but overlapping with them at the same time (Biesalski et al., 2009). Their bioavailability is partially overlapped with pharmacological and nutritional principle (21-CFR-314.3, 2016; Etcheverry et al., 2012; Heaney, 2001; Srinivasan, 2001). As shown in Fig. 1, bioavailability covers the range of bioaccessibility, metabolism and physiological activity (or simply called bioactivity) (Carbonell-Capella et al., 2014; Etcheverry et al., 2012; Gutiérrez-Grijalva et al., 2016).

As a food component, phenolics are not fully released and the released phenolics are poorly absorbed. Furthermore, the absorbed phenolic molecules cannot be completely transported to the action site in order to exert their bioactive effects. In this connection, their physicochemical properties including the degree of polymerization or glycosylation and molecular properties, polarity and interaction status with nutrients, as well as individual physiological conditions such as the expression of transport protein and status of tissues are important factors to consider. Regardless of the individual differences, the investigation on bioaccessibility and its effects provide valuable information for the selection of proper phenolic ingested dose and cooking/processing method of food (Carbonell-Capella et al., 2014). In this contribution, based on the overlap between drug and dietary phenolics, the bioavailability of phenolics involved is taken with consideration of all the pharmacokinetic steps 
of digestion, absorption, deposition, distribution, metabolism, excretion, and function.

\section{Bioaccessibility of phenolics}

\subsection{Liberation and in vitro simulated digestion}

As defined in the introduction section, bioaccessibility consists of digestibility and absorptivity. Digestion is the breakdown of large insoluble food molecules, assisted by various digestive enzymes and $\mathrm{pH}$ change, into small soluble molecules. The phenolic-matrix interaction determines phenolic liberation. For dietary phenolics, they are generally classified as free, conjugated and insolublebound phenolics (Madhujith and Shahidi, 2009). Free phenolics are present as phenolic aglycones and conjugated phenolics generally occur as phenolic glycosides, most of which are readily released in the digestive juice and absorbed as cell membrane bursts and cytoplasm diffuses into digestive juice by the effects of mechanical or chemical digestion. The insoluble-bound phenolics are covalently bound to indigestible matrices such as polysaccharides (pectin, hemicellulose, cellulose, and arabinoxylan), rod-shaped structural proteins and highly-polymerized phenolics (condensed tannin and lignin) (Acosta-Estrada et al., 2014; Shahidi and Ambigaipalan, 2016). These phenolics are partially or very marginally released, few of them penetrate through the intestinal epithelium and arrive into blood, leading to a low bioaccessibility in the upper intestinal tract (Peng et al., 2017; Shahidi and Yeo, 2016). Depending on the species and fractions of the plant, the proportion of bound phenolics varies from 20 to $90 \%$ (Acosta-Estrada et al., 2014). While only less than $10 \%$ of phenolics could get through small intestinal epithelium into circulation system and exert bioactivity at the target cell and tissue, the rest of the phenolics flow to colon with other unabsorbed residues. This part of phenolics may be metabolized and released via fermentation in the colon and could then be absorbed and further metabolized (Shahidi and Yeo, 2016). It is reported that only $2.6 \%$ of total released ferulic acids in wheat could be released by gastric and small intestinal digestion, and over $95 \%$ ferulic acids were released during colonic fermentation (Kroon et al., 1997). To understand phenolic digestion, phenolic release process includes the gastrointestinal digestion and colonic fermentation, both of which need to be emphasized. Presently, various in vitro/in vivo methods including simulated gastrointestinal digestion, simulated colon fermentation, artificial/ cultured/isolated semipermeable membrane system, Ussing chambers, animal intestinal perfusion, and animal/human pharmacokinetics studies are conducted for digestion and absorption investigations (Carbonell-Capella et al., 2014). Among them, in vitro simulated digestion process is the most widely utilized approach to predict the digestibility due to its low cost, high efficiency, and simple operation. A recent HPLC-MS analysis on lingonberries phenolic products in different digestion methods confirmed that similar metabolism patterns occurred within in vivo and in vitro digestion process which shows the reliability of in vitro digestion (Brown et al., 2014).

Several in vitro stimulated digestion results of plant foods are presented in Table 1. Based on the components studied, the simulated conditions especially the application of the enzymes and digestion time must be considered for each specific food sample. According to standardized digestion condition of Minekus et al. (2014) (Table 1), the primary simulated digestion includes a threestage process given below.

1. Simulated oral treatment with a final amylase activity of 75
$\mathrm{U} / \mathrm{mL}$, at $\mathrm{pH} 7$, for $2 \mathrm{~min}$ (volume of sample to volume of digestion fluid, 1:1)

2. Simulated gastric treatment with a final pepsin activity of $2,000 \mathrm{U} / \mathrm{mL}$, at $\mathrm{pH} \mathrm{2,} \mathrm{for} 2 \mathrm{~h}$ (volume of sample to volume of digestion fluid, $1: 1$ )

3. Simulated intestinal treatment with pancreatin based on a final trypsin activity of $100 \mathrm{U} / \mathrm{mL}$, at $\mathrm{pH} 7$, for $6 \mathrm{~h}$ (volume of sample to volume of digestion fluid, $1: 1$ ).

In addition to the most primary condition of enzymes and $\mathrm{pH}$, some standardized principles such as bile extract concentration (10 $\mathrm{mM}$ ), simulated gastrointestinal movement, body temperature, and dark anaerobic environment are also necessary. Besides, there are some other non-standardized conditions including food particle size, gastrointestinal transit time, special enzymes and the addition of some media compounds such as emulsifiers and electrolytes in simulated digestion process that also need to be considered (Hur et al., 2011; Minekus et al., 2014).

Several results about in vitro release rate of phenolics have been summarized in Tables 2 and 3. Chandrasekara and Shahidi (2012) conducted a three-stage digestion test of five millet grain samples and found gastric digestion was the major release stage for Paspalum scrobiculatum and Elusine coracana (more than $80 \%$ of total releasable phenolics), but for Setaria italic and Panicum miliacium was intestinal digestion. Due to the relatively low effects of oral treatment in the entire digestion efficiency, the three-stage digestion is sometimes simplified into two-stage without its consideration, which is more used in simulated digestion of various vegetables, fruits, beverages and other foods (Bermúdez-Soto et al., 2007; Bouayed et al., 2011; Chen et al., 2015; Faller et al., 2012; Toydemir et al., 2013; Vallejo et al., 2004).

Some studies report that the intestine is mainly responsible for the liberation of phenolics. The effect of digestion on phenolic content and antioxidant activity of 33 fruits, for phenolic release, was investigated by Chen et al. (2015). Each stage, especially intestinal digestion, was found to increase the phenolic content in the digestion fluid. Yang et al. (2018) found that the in vitro digestion treatment with bile salts was 5 times higher than the counterpart without bile salts in phenolic release. However, for antioxidant activity, it only increased during the gastric process and decreased severely in the intestinal process (Chen et al., 2015). According to the study of Chandrasekara and Shahidi (2012) on grain, flavonoids are released upon intestinal digestion better than highly-polar phenolic acids; this may be attributed to the longer release time and emulsification effect of intestinal digestion that is indispensable for relatively hydrophobic flavonoids. Furthermore, comparing the control group without $\mathrm{pH}$ variation and enzyme treatment, the counterpart with only $\mathrm{pH}$ variation (without enzymes) significantly decreased the bioaccessible phenolic content of some grains but increased their antioxidant activity. This implies that gastrointestinal digestion may not only break down food matrices and release phenolic compounds by $\mathrm{pH} / \mathrm{enzyme}$ effects but may also modify phenolic hydroxyl group (major functional group with effects on antioxidant activity) of the released phenolics which would lead to a decrease or an increase of phenolics content and activity in the final simulated digestion fluid (Table 4) (Bugianesi et al., 2004; Chen et al., 2013; Chiang et al., 2013; Hithamani and Srinivasan, 2014; Kamiloglu et al., 2015; Li et al., 2014; Sessa et al., 2011; Tagliazucchi et al., 2010; Vallejo et al., 2004).

\subsection{Absorption in the gastrointestinal tract}

Understanding the absorption of dietary phenolics is of fundamental importance in determining their biological activity. This is 
because the degree of absorption from the diet profoundly affects bioactivities at phenolics-responsive sites within the body (Day et al., 2003).

\subsubsection{Absorptivity by in vitro, in situ and in vivo methods}

The in vitro simulated digestion enables further study of gastrointestinal absorptive efficiency. For the in vitro absorptivity evaluation, the dialysis method is usually employed as a simulated intestinal epithelial cell layer to separate the pervious/absorbable fraction from the digestion fluid (Table 5). The dialysis membrane used is a semipermeable cellulose membrane with uniform pores allowing phenolics to pass via free diffusion. Based on free diffusion mechanism which is one of the main absorption pathways of phenolics, low-molecular-weight phenolics are easy to pass, and molecular weight of phenolics more than the cut-off molecular weight of dialysis membrane would be important about what is retained. The ratio of the penetrable fraction to the total content of such composition in raw materials is the predicted absorptivity. The cut-off molecular mass of this semipermeable cellulose membrane is usually around $10 \mathrm{kDa}$ (Hemery et al., 2010; Hithamani and Srinivasan, 2014; McDougall et al., 2005).

Human colon cell lines such as Caco-2 and HT-29 cells are also prevalently used in bioaccessibility assays (Hackman et al., 2008; Martin and Appel, 2009). Through in vitro simulated absorption experiments by using cell lines, the absorption efficiency and factors affecting it could be clarified. Boyer et al. (2005) used Caco-2 cell monolayer to investigate the uptake of quercetin and quercetin4 '-glucoside with/without digestion pre-treatment. Data shows that the ingested dosage affects the absorption of flavonoids. Standards of quercetin and quercetin-4'-glucoside at high concentrations resulted in higher uptake than that of diluted ones and compared to undigested fraction, the digested quercetin, and quercetin- 4 'glucoside showed a higher uptake. Besides, it was demonstrated that the lactase expressed exclusively by mammalian small intestine enterocytes could hydrolyze quercetin-4'-glucoside into its aglycone and then increase the absorption of total phenolics.

On the other hand, even if the combination of in vitro digestion and dialysis methods provides rapid and predictive data for food digestion and absorption, the actual operation of the digestive system and body physiological responses are complicated and difficult to be completely simulated. Meanwhile, the study of the correlation between in vitro and in vivo results is sometimes contradictory. For example, the absorption of quercetin glucosides in the Caco-2 cell line was fairly low compared to that of quercetin aglycone, and the apical-to-basal transporting rate of both quercetin and its glycosides are much higher than their basal-to-apical transporting rate, which shows it is unavailable for the absorption of quercetin glucosides through active transportation in Caco-2 cell lines (Walgren et al., 1998). However, it is unexpectedly found that the absorption of glycosides was higher than that of aglycones in in vivo absorption experiment conducted by human ileostomy skill (Day et al., 2003; Hollman et al., 1995). This result was confirmed in a pharmacokinetic study two years later by Hollman et al. (1997). The main reason for this observation was regarded to be active transporting of glycosides. Moreover, the pharmacokinetic study of anthocyanins showed that only about $1 \%$ could reach systematic circulation, while the cellular study reported that $3-4 \%$ of them got through human intestinal cell monolayers (Faria et al., 2009; Lee et al., 2014). In simulated absorption test by Caco-2 monolayer, the proanthocyanidin trimers could be absorbed and showed the same permeability coefficients with catechin, proanthocyanidin dimer, and mannitol, while in an animal model, there was no actual absorption of proanthocyanidin trimers (Appeldoorn et al., 2009; Deprez et al., 2001).

On the basis of the preceding and other available literature, animal models and human experiments are essential as they provide accurate information. The study of in vitro absorption of catechin and tannic acid in ligated rat small intestine segments showed that both tannic acid $(50 \%)$ and catechin $(30 \%)$ could enter into the small intestinal cells, but only catechin $(10 \%)$ passed through the gut wall and arrived into the incubation buffers whilst no tannic acid was detected (Carbonaro et al., 2001). In the human experiment, $53 \%$ of ingested quercetin $(4 \mathrm{~g})$ and $98 \%$ of chrysin $(0.4 \mathrm{~g})$ were detected in the feces (Gugler et al., 1975; Walle et al., 2001). Genistein absorption was also investigated by using rat small intestine perfusion model in which genistein solution was perfused into isolated rat small intestine; genistein was recovered from vascular perfusion media, blood vessel, intestine tissue, and non-absorbable effluent. The results indicated $99.8 \%$ recovery, and $46 \%$ of genistein could be absorbed. Among absorbed genistein, about $40 \%$ of it came from vascular perfusion media, $6 \%$ was from blood vessels and the intestinal tissue (Andlauer et al., 2000). Griffiths and Smith (1972) and Booth et al. (1956) reported that the C-ring cleavage caused by intestinal bacterial flora had a preference for flavonoids with hydroxyl group in positions 5 and 4, such as apigenin, genistein, quercetin, rutin, kaempferol, robinin, and pelargonin, but on the contrary, apigenin 4-methyl ether, 4',7-dihydroxyflavone, daidzein, 5-methoxyquercetin, chrysin, tectochrysin, biochanin A and formononetin were relatively resistant to ring fission. However, Andlauer et al. (2000) reported that no cleavage happened on genistein during small intestinal absorption. Thus the intestinal microbial ring fission effects should have only happened in the large intestine. The absorption of phenolic oligomers depends on their degree of polymerization; some with low-polymerization degree could be absorbed in the stomach and the small intestine by passive diffusion, others with a high-polymerization degree could not be absorbed until undergoing fermentation and degradation by colonic bacteria. For example, monoferluate ester and diferulate ester standards could be absorbed in rat upper intestine, and ferulic acid could be absorbed by gastric mucosa (Andreasen et al., 2001b). However, procyanidin A-type trimers and tetramers were absorbed by small intestine in the rat perfusion model, instead of which, procyanidin dimers A1, A2, and B2 were available to be absorbed. Meanwhile, the absorptivity of all dimers was extremely low, only about $5-10 \%$ of that of epicatechin, even though A1 and A2 were absorbed better than B2 (Appeldoorn et al., 2009). As Manach et al. (2005) summarized, the orally administrated anthocyanins presented the lowest gastrointestinal absorbability $(0.004-5.1 \%)$ compared to other phenolics such as isoflavones (4-62\%), flavanones (1.1-30.2\%), flavonols $(0.07-7 \%)$, flavanols $(0.02-55 \%)$ and phenolic acids $(0.3-61.7 \%)$.

\subsubsection{Influx transport of enterocytes}

The uptake of phenolics occurs in a highly complex manner through multiple pathways. Normally, similar to all fat-soluble micromolecules, the relatively neutral or hydrophobic phenolics such as phenolic lipids, proanthocyanidins, artificial phenolic esters, (iso)flavonoid and lignan aglycones could be passively diffused through apical membrane of the epithelial cell (Domínguez-Avila et al., 2017; Kobayashi et al., 2013). The penetration efficiency is decided by their lipophilicity. For example, the revealed affinity of catechin derivatives to the lipid bilayer decreased in the order of ECG > EGCG > EC > EGC (Tarahovsky et al., 2014). Generally, a lower $\mathrm{pH}$ suppresses ionization of phenolics and thus favors a 
Table 1. Comparison of in vitro digestion method in bioaccessibility determination

\begin{tabular}{|c|c|c|c|c|}
\hline Materials & Oral condition & Gastric condition & Small intestine condition & References \\
\hline \multicolumn{5}{|l|}{ A Standardized Condition } \\
\hline Food Sample & $\begin{array}{l}1: 1(\mathrm{v} / \mathrm{v}) \sim \text { Oral fluid } \\
\text { (amylase-75 U/ } \\
\mathrm{mL} \text { ): Sample, } \mathrm{pH} \\
7.0,2 \mathrm{~min}, 37^{\circ} \mathrm{C}\end{array}$ & $\begin{array}{l}\text { 1:1 (v/v) Gastric } \\
\text { fluid (pepsin-2,000 } \\
\text { U/mL): Oral fluid, } \\
\mathrm{pH} \mathrm{3.0,} 2 \mathrm{~h}, 37^{\circ} \mathrm{C}\end{array}$ & $\begin{array}{l}1: 1(\mathrm{v} / \mathrm{v}) \sim \text { Intestinal fuild } \\
\text { (pancreatin based on } \\
\text { trypsin activity at } 100 \mathrm{U} / \\
\mathrm{mL} \text {, bile salts- } 10 \mathrm{mM} \text { ): Gatric } \\
\text { fraction, } \mathrm{pH} 7.5,6 \mathrm{~h}, 37^{\circ} \mathrm{C}\end{array}$ & $\begin{array}{l}\text { Minekus et } \\
\text { al. (2014) }\end{array}$ \\
\hline \multicolumn{5}{|c|}{ Phenolics and Other phytochemicals } \\
\hline Tea beverage (phenolics) & & $\begin{array}{l}\text { Pepsin, pH 2.0, } \\
1 \mathrm{~h}, 37^{\circ} \mathrm{C}\end{array}$ & $\begin{array}{l}\text { Pancreatin, Bile salts } \\
\text { (glycodeoxycholate, } \\
\text { taurodeoxycholate, } \\
\text { taurocholate), pH } 7.4 \text { (transition } \\
\text { pH 5.3), } 2.5 \mathrm{~h}, 37^{\circ} \mathrm{C}\end{array}$ & $\begin{array}{l}\text { Chen et al. } \\
(2013)\end{array}$ \\
\hline $\begin{array}{l}\text { Grape seed extract } \\
\text { (flavonoids) }\end{array}$ & $\begin{array}{l}\text { Human saliva } \alpha \text { - } \\
\text { Amylase in water } \\
\text { with weak acid, } \mathrm{pH} \\
6.9,10 \mathrm{~min}, 37^{\circ} \mathrm{C}\end{array}$ & $\begin{array}{l}\text { Pepsin, pH 2.0, } \\
1 \mathrm{~h}, 37^{\circ} \mathrm{C}\end{array}$ & $\begin{array}{l}\text { Pancreatin, Bile salts, } \mathrm{pH} 7.0 \\
\text { (transition } \mathrm{pH} 6.0 \text { ), } 2 \mathrm{~h}, 37^{\circ} \mathrm{C}\end{array}$ & $\begin{array}{l}\text { Laurent et } \\
\text { al. (2007) }\end{array}$ \\
\hline Wheat bread (flavonoids) & $\begin{array}{l}\text { Human saliva in buffer } \\
\left(\mathrm{Na}_{2} \mathrm{HPO}_{4}, \mathrm{KH}_{2} \mathrm{PO}_{4},\right. \\
\text { and } \mathrm{NaCl}), \mathrm{pH}^{7.0} \text {, } \\
10 \text { min, } 37^{\circ} \mathrm{C}\end{array}$ & $\begin{array}{l}\text { Pepsin, pH 1.2, } \\
2 \mathrm{~h}, 37^{\circ} \mathrm{C}\end{array}$ & $\begin{array}{l}\text { Pancreatin, Bile salts, } \mathrm{NaCl} \text {, } \\
\mathrm{KCl}, \mathrm{pH} 7.0 \text { (transition } \\
\mathrm{pH} \mathrm{6.0),} 1 \mathrm{~h}, 37^{\circ} \mathrm{C}\end{array}$ & $\begin{array}{l}\text { Gawlik-Dziki } \\
\text { et al. (2009) }\end{array}$ \\
\hline Soy bread (isoflavones) & $\begin{array}{l}\text { Human saliva in saline, } \\
\mathrm{pH} 7.0,5 \mathrm{~min}, 37^{\circ} \mathrm{C}\end{array}$ & $\begin{array}{l}\text { Pepsin, pH 2.0, } \\
1 \mathrm{~h}, 37^{\circ} \mathrm{C}\end{array}$ & Pancreatin, pH 6.9, $2 \mathrm{~h}, 37^{\circ} \mathrm{C}$ & $\begin{array}{l}\text { Walsh et al. } \\
\text { (2003) }\end{array}$ \\
\hline Millet grain (phenolics) & $\begin{array}{l}\text { Porcine } \alpha \text {-amylase } \\
\text { in phosphate buffer, } \\
\mathrm{pH} 6.9,5 \mathrm{~min}, 37^{\circ} \mathrm{C}\end{array}$ & $\begin{array}{l}\text { Pepsin, } \mathrm{pH} 2.5 \text {, } \\
2 \mathrm{~h}, 37^{\circ} \mathrm{C}\end{array}$ & $\begin{array}{l}\text { Pancreatin, Mucin, Bile } \\
\text { salts, } \mathrm{pH} 7.0,3 \mathrm{~h}, 37^{\circ} \mathrm{C}\end{array}$ & $\begin{array}{l}\text { Chandrasekara } \\
\text { et al. (2012) }\end{array}$ \\
\hline Raspberry (anthocyanins) & & $\begin{array}{l}\text { Pepsin in water, } \mathrm{pH} \\
1.7,2 \mathrm{~h}, 37^{\circ} \mathrm{C}\end{array}$ & $\begin{array}{l}\text { Pancreatin, Bile salts, } \\
\mathrm{pH} 7.0,2 \mathrm{~h}, 37^{\circ} \mathrm{C}\end{array}$ & $\begin{array}{l}\text { McDougall et } \\
\text { al. (2005) }\end{array}$ \\
\hline Blueberry (phenolics) & & $\begin{array}{l}\text { Pepsin in water, } \mathrm{pH} \\
2.0,2 \mathrm{~h}, 37^{\circ} \mathrm{C}\end{array}$ & $\begin{array}{l}\text { Pancreatin, Bile salts, } \\
\mathrm{pH} 7.5,2 \mathrm{~h}, 37^{\circ} \mathrm{C}\end{array}$ & $\begin{array}{l}\text { Correa-Betanzo } \\
\text { et al. (2014) }\end{array}$ \\
\hline Gooseberry (phenolics) & & $\begin{array}{l}\text { Pepsin in water, } \mathrm{pH} \\
1.7-2.0,2 \mathrm{~h}, 37^{\circ} \mathrm{C}\end{array}$ & $\begin{array}{l}\text { Pancreatin, Bile salts } \\
\text { (glycodeoxycholate, } \\
\text { taurodeoxycholate, } \\
\text { taurocholate), pH 8.0, } \\
\text { overnight, } 37^{\circ} \mathrm{C}\end{array}$ & $\begin{array}{l}\text { Chiang et } \\
\text { al. (2013) }\end{array}$ \\
\hline $\begin{array}{l}\text { Various baby food } \\
\text { (carotenoids) }\end{array}$ & & $\begin{array}{l}\text { Pepsin in water, } \mathrm{pH} \\
2.0,1 \mathrm{~h}, 37^{\circ} \mathrm{C}\end{array}$ & $\begin{array}{l}\text { Pancreatin, Bile salts, } \mathrm{pH} 7.5 \\
\text { (transition } \mathrm{pH} 5.3 \text { ) } 2 \mathrm{~h}, 37^{\circ} \mathrm{C}\end{array}$ & $\begin{array}{l}\text { Garrett et } \\
\text { al. (1999) }\end{array}$ \\
\hline $\begin{array}{l}\text { Various food (carotenoids } \\
\text { and tocopherol) }\end{array}$ & & $\begin{array}{l}\text { Pepsin in saline, } \\
\text { Pyrogallol, } \mathrm{pH} \\
4.0,1 \mathrm{~h}, 37^{\circ} \mathrm{C}\end{array}$ & $\begin{array}{l}\text { Pancreatin, Bile salts, } \\
\mathrm{pH} 6.0,0.5 \mathrm{~h}, 37^{\circ} \mathrm{C}\end{array}$ & $\begin{array}{l}\text { Reboul et } \\
\text { al. (2006) }\end{array}$ \\
\hline $\begin{array}{l}\text { Various food (tocopherol } \\
\text { and retinol) }\end{array}$ & & $\begin{array}{l}\text { Pepsin in HBSS, lipase, } \\
\mathrm{pH} 4,1 \mathrm{~h}, 37^{\circ} \mathrm{C}\end{array}$ & $\begin{array}{l}\text { Pancreatin, Bile salt } \\
\text { (glycodeoxycholate, taurocholate, } \\
\text { and taurodeoxycholate), } \mathrm{pH} \\
7.8 \text { (transit } \mathrm{pH} 5.4 \text { ), } 2 \mathrm{~h}, 37^{\circ} \mathrm{C}\end{array}$ & $\begin{array}{l}\text { O'Callaghan } \\
\text { et al. (2010) }\end{array}$ \\
\hline $\begin{array}{l}\text { Broccoli (phenolics, } \\
\text { glucosinolates, } \\
\text { ascorbic acid) }\end{array}$ & & $\begin{array}{l}\text { Porcine pepsin in } \\
\text { water, } \mathrm{pH} 2,2 \mathrm{~h}, 37^{\circ} \mathrm{C}\end{array}$ & $\begin{array}{l}\text { Pancreatin, Lipase, Bile } \\
\text { salts, } \mathrm{pH} 7,2 \mathrm{~h}, 37^{\circ} \mathrm{C}\end{array}$ & $\begin{array}{l}\text { Vallejo et } \\
\text { al. (2004) }\end{array}$ \\
\hline $\begin{array}{l}\text { Hemi-purified } \\
\text { glucoraphanin }\end{array}$ & $\begin{array}{l}\alpha \text {-Amylase in water, } \\
\mathrm{pH} 7.0,3 \mathrm{~min}, 37^{\circ} \mathrm{C}\end{array}$ & Pepsin, $\mathrm{pH} 2,2 \mathrm{~h}, 37^{\circ} \mathrm{C}$ & $\begin{array}{l}\text { Pancreatin, Bile salts, } \\
\mathrm{pH} 7.5,2 \mathrm{~h}, 37^{\circ} \mathrm{C}\end{array}$ & Lai et al. (2010) \\
\hline Soy flour (saponins) & $\begin{array}{l}\alpha \text {-Amylase in PBS, } \mathrm{pH} \\
7.0,10 \mathrm{~min}, 37^{\circ} \mathrm{C}\end{array}$ & $\begin{array}{l}\text { Pepsin, pH 2.5, } \\
2 \mathrm{~h}, 37^{\circ} \mathrm{C}\end{array}$ & $\begin{array}{l}\text { Pancreatin, Lipase, Bile salt } \\
\text { (glycodeoxycholate, taurocholate, } \\
\text { and taurodeoxycholate), } \\
\mathrm{pH} 6.5,2 \mathrm{~h}, 37^{\circ} \mathrm{C}\end{array}$ & $\begin{array}{l}\text { Serventi et } \\
\text { al. (2013) }\end{array}$ \\
\hline
\end{tabular}


Table 1. Comparison of in vitro digestion method in bioaccessibility determination - (continued)

\begin{tabular}{|c|c|c|c|c|}
\hline Materials & Oral condition & Gastric condition & Small intestine condition & References \\
\hline $\begin{array}{l}\text { Milk-based fruit } \\
\text { beverage (phytosterol) }\end{array}$ & $\begin{array}{l}\alpha \text {-Amylase, Mucin, } \\
\mathrm{pH} 6.5,5 \mathrm{~min}, 37{ }^{\circ} \mathrm{C}\end{array}$ & $\begin{array}{l}\text { Pepsin, BSA, pH } \\
1.07,1 \mathrm{~h}, 37^{\circ} \mathrm{C}\end{array}$ & $\begin{array}{l}\text { Pancreatic lipase, Cholesterol } \\
\text { esterase, Phospholipase } \\
\text { A2, Colipase, Taurocholate, } \\
\mathrm{pH} 7.0,2 \mathrm{~h}, 37^{\circ} \mathrm{C}\end{array}$ & $\begin{array}{l}\text { Alvarez-Sala } \\
\text { et al. (2016) }\end{array}$ \\
\hline
\end{tabular}

\section{Other Ingredients}

Triacylglycerol (cod liver oil)

$\begin{array}{ll} & 2.0 \text { (transition } \mathrm{pH} \\ & 4.0 \text { for another } 30 \\ & \min ), 0.5 \mathrm{~h}, 37^{\circ} \mathrm{C}\end{array}$

\section{Starch (variously}

modified starch and

unmodified starch)

Proteins (spelt products)

Polysaccharides (seeds

of Plantago asiatica L.) $\mathrm{pH} 7.0,4 \mathrm{~h}, 37^{\circ} \mathrm{C}$

\begin{abstract}
Lipase, Pepsin, $\mathrm{pH}$
$\min ), 0.5 \mathrm{~h}, 37^{\circ} \mathrm{C}$
\end{abstract}

Pepsin, pH 2.0,

$0.5 \mathrm{~h}, 37^{\circ} \mathrm{C}$

Pepsin, pH 1.9,

$0.5 \mathrm{~h}, 37^{\circ} \mathrm{C}$

Pepsin, Lipase, Gastric

Electrolytes ( $\mathrm{NaCl}, \mathrm{KCl}$, $\mathrm{CaCl}_{2} \cdot 2 \mathrm{H}_{2} \mathrm{O}, \mathrm{NaHCO}_{3}$ ), $\mathrm{pH} 3.0,6 \mathrm{~h}, 37^{\circ} \mathrm{C}$

Iron (rye bread with $\mathrm{FeCl}_{3}$ )
Pepsin in saline, L-lactic acid, Inositol phosphates, $\mathrm{pH}$ 2.0, $1 \mathrm{~h}, 37^{\circ} \mathrm{C}$

\author{
Pancreatin, Bile \\ salts (glycocholate, \\ glycochenodeoxycholate, \\ glycodeoxycholate, taurocholate, \\ taurochenodeoxycholate, \\ taurodeoxycholate, \\ taurolithocholate)
} Larsson et

\section{Pancreatin, $\alpha$-Amylase, Amyloglucosidase, Acetate buffer, $\mathrm{pH} 5.0,37^{\circ} \mathrm{C}$}

Pancreatin in Phosphate

Pancreatin, Trypsin, Bile

salts, Small intestinal

electrolytes ( $\mathrm{NaCl}, \mathrm{CaCl}_{2} \cdot 2 \mathrm{H}_{2} \mathrm{O}$ ), $\mathrm{pH} 7.0,6 \mathrm{~h}, 37^{\circ} \mathrm{C}$
Pancreatin, Saline, $\mathrm{pH}$
7.5, $6 \mathrm{~h}, 37^{\circ} \mathrm{C}$

Abdel-Aal (2008)

Bering et al.

(2006)

Wolf et al. (1999)

Hu et al. (2013)

(2006)

$$
\text { al. (2012) }
$$


Table 2. Release evaluation of phenolics during in vitro digestion

\begin{tabular}{|c|c|c|c|c|c|c|}
\hline \multirow{2}{*}{ Sample } & \multirow{2}{*}{ Oral phase } & \multicolumn{2}{|c|}{ Release percentage of phenolics (\%) } & \multirow{2}{*}{$\begin{array}{l}\text { Release } \\
\text { standard }\end{array}$} & \multirow{2}{*}{$\begin{array}{l}\text { Total phenolics } \\
\text { extraction }\end{array}$} & \multirow{2}{*}{ References } \\
\hline & & Gastric phase & Intestinal phase & & & \\
\hline $\begin{array}{l}\text { Apple } \\
\text { homogenate }\end{array}$ & & $\begin{array}{l}\text { Phenolics (68) } \\
\text { Flavonoids (65) } \\
\text { Anthocyanins (91) }\end{array}$ & $\begin{array}{l}\text { Phenolics (74) } \\
\text { Flavonoids (72) } \\
\text { Anthocyanins }\end{array}$ & Solubility & $\begin{array}{l}\text { Pure methanol } \\
\text { Ultrasonication }\end{array}$ & $\begin{array}{l}\text { (Bouayed et } \\
\text { al., 2011) }\end{array}$ \\
\hline
\end{tabular}

\begin{tabular}{|c|c|c|c|c|c|c|}
\hline & & \multicolumn{2}{|c|}{$\begin{array}{l}\text { Phenolics (54) } \\
\text { Flavonoids (39) } \\
\text { Anthocyanins (0) }\end{array}$} & Dialyzability & & \\
\hline \multirow[t]{2}{*}{ Orange juice } & & $\begin{array}{l}\text { Flavanones (51) } \\
\text { Vicenin-2 (117) }\end{array}$ & $\begin{array}{l}\text { Flavanones (51) } \\
\text { Vicenin-2 (111) }\end{array}$ & Solubility & $\begin{array}{l}\text { Direct } \\
\text { determination }\end{array}$ & $\begin{array}{l}\text { Gil-Izquierdo } \\
\text { et al. (2001) }\end{array}$ \\
\hline & & \multicolumn{2}{|c|}{$\begin{array}{l}\text { Flavonones (6) } \\
\text { Vincenin-2 (22) }\end{array}$} & Dialyzability & & \\
\hline $\begin{array}{l}\text { Pumpkin } \\
\text { flour }\end{array}$ & & \multicolumn{2}{|c|}{ Phenolics (29-37) } & Solubility & $\begin{array}{l}\text { Free: } \mathrm{HCl} \text {, water, } \\
\text { and methanol } \\
(1: 80: 10) ; \\
\text { Bound: } \mathrm{H}_{2} \mathrm{SO}_{4} \\
\text { and methanol } \\
(1: 10)\end{array}$ & $\begin{array}{l}\text { Aydin and } \\
\text { Gocmen. } \\
(2015)\end{array}$ \\
\hline Grape & $\begin{array}{l}50 \% \text { phenolics } \\
27 \% \text { flavonoids } \\
19 \% \text { anthocyanins }\end{array}$ & $\begin{array}{l}\text { Phenolics (61) } \\
\text { Flavonoids (43) } \\
\text { Anthocyanins (36) }\end{array}$ & $\begin{array}{l}\text { Phenolics (62) } \\
\text { Flavonoids (56) } \\
\text { Anthocyanins (8) }\end{array}$ & Solubility & $\begin{array}{l}\text { Free: acidified } \\
\text { water } \\
\text { Bound: acidified } \\
\text { methanol }\end{array}$ & $\begin{array}{l}\text { Tagliazucchi } \\
\text { et al. (2010) }\end{array}$ \\
\hline Green lentil & & $\begin{array}{l}\text { Phenolics (21) } \\
\text { Flavonoids (29) }\end{array}$ & $\begin{array}{l}\text { Phenolics (50) } \\
\text { Flavonoids (71) }\end{array}$ & Solubility & $\begin{array}{l}\text { Acidified } 70 \% \\
\text { methanol }\end{array}$ & $\begin{array}{l}\text { Zhang et } \\
\text { al. (2017) }\end{array}$ \\
\hline $\begin{array}{l}\text { Grape } \\
\text { pomace }\end{array}$ & & Phenolics (102) & Phenolics (67) & Solubility & $\begin{array}{l}\text { Acidified pure } \\
\text { methanol; } \\
70 \% \text { acetone }\end{array}$ & $\begin{array}{l}\text { Wang et } \\
\text { al. (2017) }\end{array}$ \\
\hline $\begin{array}{l}\text { Globe } \\
\text { artichoke }\end{array}$ & $\begin{array}{l}27 \% \text { caffeoylquinic } \\
\text { acid and } \\
\text { dicaffeoylquinic } \\
\text { acid }\end{array}$ & $\begin{array}{l}\text { Caffeoylquinic acid } \\
\text { and dicaffeoylquinic } \\
\text { acid (36) }\end{array}$ & $\begin{array}{l}\text { Caffeoylquinic acid } \\
\text { and dicaffeoylquinic } \\
\text { acid (55.8) }\end{array}$ & Solubility & $100 \%$ Water & $\begin{array}{l}\text { D'Antuono } \\
\text { et al. (2015) }\end{array}$ \\
\hline Pili pomace & & $\begin{array}{l}\text { Phenolics (12) } \\
\text { Flavonoids (0.7) } \\
\text { Tannins (3.2) } \\
\text { Anthocyanins (200) }\end{array}$ & $\begin{array}{l}\text { Phenolics (6) } \\
\text { Flavonoids (0.2) } \\
\text { Tannins (1.2) } \\
\text { Anthocyanins (10) }\end{array}$ & Solubility & $\begin{array}{l}\text { Acidified } 50 \% \\
\text { ethanol }\end{array}$ & $\begin{array}{l}\text { Arenas and } \\
\text { Trinidad } \\
(2017)\end{array}$ \\
\hline $\begin{array}{l}\text { Black bean } \\
\text { coat }\end{array}$ & & \multicolumn{2}{|c|}{$\begin{array}{l}\text { Phenolics (24) } \\
\text { Flavonoids (82) } \\
\text { Tannins (6) }\end{array}$} & Solubility & \multirow{2}{*}{$\begin{array}{l}\text { Acidified } \\
\text { pure water } \\
\text { Acidified pure } \\
\text { methanol }\end{array}$} & $\begin{array}{l}\text { Sancho et } \\
\text { al. (2015) }\end{array}$ \\
\hline $\begin{array}{l}\text { Small red } \\
\text { bean coat }\end{array}$ & & \multicolumn{2}{|c|}{$\begin{array}{l}\text { Phenolics (49) } \\
\text { Flavonoids (95) } \\
\text { Tannins (7) }\end{array}$} & & & \\
\hline Kale & & \multicolumn{2}{|c|}{ Phenolics (69) } & Solubility & $\begin{array}{l}\text { Hexane; } \\
\text { Acetone; } \\
\text { Methanol, water } \\
\text { and formic } \\
\text { acid }(80: 19: 1)\end{array}$ & $\begin{array}{l}\text { Yang et al. } \\
\text { (2018) }\end{array}$ \\
\hline
\end{tabular}

$\left.\mathrm{mM}^{-1} \mathrm{~s}^{-1}\right)$ and lactose $\left(4 \mathrm{mM}^{-1} \mathrm{~s}^{-1}\right)$ (Day et al., 2000b). This enzyme is more important for humans than other mammals in the digestion of $\beta$-glycosides because of the absence of gastric microorganism and other hydrolysis enzymes in the human intestinal tract (Day et al., 2000b).

Hollman et al. (1995) indirectly demonstrated that SGLT1, which is the glucose transporter highly expressed on the apical membrane of small intestinal epithelial cells and proximal tubule of the nephron, was responsible for glucose/galactose absorption and reabsorption. This transporter may actively favor the absorption of monoglycoside phenolics due to its preferred transportation of glucose/galactose moiety. As a result, monoglycoside phenolics 
Table 3. Effects of ingested quantity on anthocyanins bioaccessibility in black carrot pomace enriched cakes

\begin{tabular}{|c|c|c|c|c|c|}
\hline \multirow{2}{*}{ Sample (mg/g, dw) } & & \multicolumn{2}{|c|}{ Released anthocyanin (\%) } & \multirow{2}{*}{ Total phenolics extraction } & \multirow{2}{*}{ References } \\
\hline & & Gastric phase & Intestinal phase & & \\
\hline \multirow{3}{*}{$\begin{array}{l}\text { Cyanidin-3-xylosyl- } \\
\text { glucosyl-galactoside }\end{array}$} & $5(C 3 G)^{a}$ & 40.0 & 32.0 & \multirow{15}{*}{$\begin{array}{l}70 \% \text { methanol with } 0.1 \% \\
\text { formic acid; Ultrasonication }\end{array}$} & \multirow[t]{15}{*}{ Kamiloglu et al. (2017) } \\
\hline & 12 (C3G) & 75.0 & 41.7 & & \\
\hline & 20 (C3G) & 55.0 & 30.0 & & \\
\hline \multirow[t]{3}{*}{ Cyanidin-3-xylosyl-galactoside } & 19 (C3G) & 62.9 & 11.6 & & \\
\hline & $52(C 3 G)$ & 38.5 & 21.2 & & \\
\hline & 75 (C3G) & 30.7 & 18.7 & & \\
\hline \multirow{3}{*}{$\begin{array}{l}\text { Cyanidin-3-xylosyl-sinapoyl- } \\
\text { glucosyl-galactoside }\end{array}$} & 11 (C3G) & 27.3 & 45.5 & & \\
\hline & $27(C 3 G)$ & 48.1 & 44.4 & & \\
\hline & 48 (C3G) & 39.6 & 31.2 & & \\
\hline \multirow{3}{*}{$\begin{array}{l}\text { Cyanidin-3-xylosyl-feruloyl- } \\
\text { glucosyl-galactoside }\end{array}$} & 32 (C3G) & 29.2 & 40.6 & & \\
\hline & 77 (C3G) & 57.1 & 42.9 & & \\
\hline & $\begin{array}{l}111 \\
(\mathrm{C} 3 \mathrm{G})\end{array}$ & 49.5 & 46.8 & & \\
\hline \multirow{3}{*}{$\begin{array}{l}\text { Cyanidin-3-xylosyl-coumaroyl- } \\
\text { glucosyl-galactoside }\end{array}$} & $5(\mathrm{C} 3 \mathrm{G})$ & 20.0 & 40.0 & & \\
\hline & 13 (C3G) & 46.2 & 30.8 & & \\
\hline & 13 (C3G) & 61.5 & 61.5 & & \\
\hline
\end{tabular}

${ }^{\mathrm{a} C}$ 3G: cyaniding-3-O-glucoside equivalent.

present a faster and higher absorption rate than other phenolics without active transportation, and are regarded as the competitive substrates for glucose transport by SGLT1 at the same time (Cermak et al., 2004; Gee et al., 2000; Goto et al., 2012; Hollman et al., 1995). Meanwhile, Hossain et al. (2002) found that non-glycosylated phenolics such as catechin, epicatechin gallate (ECG) and epigallocatechin gallate (EGCG) also inhibited SGLT 1 response of glucose/galactose absorption. Moreover, the transportability for monoglycoside phenolics depends on their specific structure. The transport of quercetin-3/4'-O-glucoside by SGLT 1 is available while for that of non-glucosylated phenolics and some other monoglycoside phenolics (naringenin-7-O-glucoside and genistein-7-O-glucoside) may not be (Cermak et al., 2004). In the intestine, LPH protrudes into an unstirred boundary layer and is positioned in close proximity to the SGLT 1. An animal experiment was conducted to verify absorption pathway of quercetin by Day et al. (2003) who utilized inhibitors to block each pathway, respectively, and proved both of these two pathways: active transport by SGLT 1 and passive diffusion by LPH, as being responsible for absorption of quercetin glucoside. Meanwhile, data shows the pathway of flavonoid glycosides with different structures is varied, quercetin-4'-glucoside is absorbed by both interacting with SGLT 1 and luminal hydrolysis by LPH, while absorption of quercetin3 -glucoside involves only LPH. This result was also confirmed in in situ rat perfusion model that LPH was predominantly $(>75 \%)$ involved in the absorption of quercetin-3-glucoside in the small intestine (Sesink et al., 2003). However, within various glycoside forms including $\mathrm{C}-\mathrm{O}$ glycosides such as glucosides, galactosides, arabinosides, xylosides and rhamnosides, and $\mathrm{C}-\mathrm{C}$ glycosides conjugating with flavonoids, only $\beta$-glucosides could effectively be hydrolyzed by LPH. Meanwhile, low or even none hydrolysis extent was found during the absorption of daidzein-7-glucoside $\left(14 \mathrm{mM}^{-1} \mathrm{~s}^{-1}\right)$ and cyanidin-3-glucoside $\left(0 \mathrm{mM}^{-1} \mathrm{~s}^{-1}\right)$, compared with quercetin-4'-glucoside $\left(170 \mathrm{mM}^{-1} \mathrm{~s}^{-1}\right)$, quercetin-3-glucoside $\left(137 \mathrm{mM}^{-1} \mathrm{~s}^{-1}\right)$, and genistein-7-glucoside $\left(77 \mathrm{mM}^{-1} \mathrm{~s}^{-1}\right)$, even though all of them are $\beta$-glucosides (Day et al., 2000b; Hollman, 2004). The GLUTs protein family belongs to the major facilitator superfamily (MFS) of membrane transporters; they are unidirectional transporters which favor not only glucose but also phenolic aglycones and/or phenolic glycosides to enter into the cells by facilitated transport (Passamonti et al., 2009). GLUT 2 is highly expressed on the basal side of the intestinal cell, which was reported to transport quercetin-3-O-glucoside out of the basal side of Caco2 cells, but it is unavailable for ECG (Chen et al., 2007). Faria et al. (2009) reported that anthocyanins inhibit the uptake of glucose by upgrading the expression of GLUT 2. Besides, combined with other GLUT members (GLUT 1 and 4), the flavonoid aglycones and non-glycosylated polyphenols are regarded as competitive substrates for each other in facilitated transport by GLUTs, similar to the situation that flavonoid glycosides inhibit glucose absorption by SGLT 1 (Ashong et al., 2012; Faria et al., 2009).

Besides LPH, cinnamoyl esterases may provide another vital route for the release of phenolic aglycones from ester bond of polysaccharides and phenolic polymers during small intestinal digestion. Cinnamoyl esterases are well known as gut microbial enzymes excreted during colonic fermentation, but it has also been found in both small intestinal mucosa cell and lumen (Andreasen et al., 2001a). The presence of esterase activity in these sections of the intestinal tract was found in which ferulic and $p$-coumaric acids were released from spinach cell walls and might be absorbed through the stomach and small intestine of rat (Buchanan et al., 1996). This thought was verified 5 years later by extracting cinnamoyl esterases from the surface of the rat and human small intestine. The phenolic acids esters especially methyl hydroxycinnamoyl esters (monoferluate ester, diferulate ester, $p$-coumaric acid, methyl ferulate, methyl caffeate, and methyl sinapate) ex- 
Table 4. Stability of phenolic compounds during gastrointestinal digestion

\begin{tabular}{|c|c|c|c|c|}
\hline \multirow{2}{*}{\multicolumn{2}{|c|}{ Digested material }} & \multicolumn{2}{|c|}{ Phenolics loss (\%) } & \multirow{2}{*}{ References } \\
\hline & & \multirow[t]{2}{*}{ Gastric phase } & \multirow[t]{2}{*}{ Intestinal phase } & \\
\hline \multicolumn{3}{|l|}{ Phenolic standards } & & \\
\hline \multicolumn{2}{|l|}{ Gallic acid $(35.0 \mu \mathrm{g} / \mathrm{mL})$} & $4.6^{\mathrm{a}}$ & 43.3 & \multirow{5}{*}{$\begin{array}{l}\text { Tagliazucchi et } \\
\text { al. (2010) }\end{array}$} \\
\hline \multicolumn{2}{|l|}{ Caffeic acid $(8.0 \mu \mathrm{g} / \mathrm{mL})$} & $0.1^{\mathrm{a}}$ & 24.9 & \\
\hline \multicolumn{2}{|l|}{ Catechin $(40.0 \mu \mathrm{g} / \mathrm{mL})$} & $0.7^{a}$ & 7.2 & \\
\hline \multicolumn{2}{|l|}{ Quercetin $(60.0 \mu \mathrm{g} / \mathrm{mL})$} & 0.9 & 5.8 & \\
\hline \multicolumn{2}{|l|}{ Resveratrol $(3.0 \mu \mathrm{g} / \mathrm{mL})$} & -2.3 & 69.5 & \\
\hline \multicolumn{2}{|c|}{ Cyanidin 3-rutinoside (52.8 $\mu \mathrm{g} / \mathrm{mL})$} & 0 & 9.1 & \multirow{4}{*}{$\begin{array}{l}\text { Bermúdez-Soto } \\
\text { et al. (2007) }\end{array}$} \\
\hline \multicolumn{2}{|c|}{ Quercetin-3-rutinoside (160.6 g/mL) } & 0 & 3.1 & \\
\hline \multicolumn{2}{|l|}{ (+)-Catechin $(320.0 \mu \mathrm{g} / \mathrm{mL})$} & 3.1 & 58.0 & \\
\hline \multicolumn{2}{|l|}{ Chlorogenic acid $(90.0 \mu \mathrm{g} / \mathrm{mL})$} & 0 & 5.1 & \\
\hline \multicolumn{2}{|l|}{ Chlorogenic acid (100.0 g/mL) } & & 48.1 & \multirow{3}{*}{$\begin{array}{l}\text { D'Antuono et } \\
\text { al. (2015) }\end{array}$} \\
\hline \multicolumn{2}{|c|}{ 1,5-O-Dicaffeoylquinic acid $(100.0 \mu \mathrm{g} / \mathrm{mL})$} & & 49.6 & \\
\hline \multicolumn{2}{|c|}{ 3,5-O-Dicaffeoylquinic acid $(100.0 \mu \mathrm{g} / \mathrm{mL})$} & & 25.8 & \\
\hline \multicolumn{2}{|l|}{ Chlorogenic acid (67.5 $\mu \mathrm{g} / \mathrm{mL})$} & 58.1 & 95.7 & Siracusa et \\
\hline \multicolumn{2}{|l|}{ Rutin $(45.0 \mu \mathrm{g} / \mathrm{mL})$} & 88.1 & total & al. (2011) \\
\hline \multicolumn{2}{|c|}{ Quercetin 3-O-glucoside $(30.0 \mu \mathrm{g} / \mathrm{mL})$} & total & total & \\
\hline \multicolumn{2}{|l|}{ Quercetin $(15.0 \mu \mathrm{g} / \mathrm{mL})$} & total & total & \\
\hline Pelargonidin-3-glucoside & & 1.0 & 19.0 & Woodward et \\
\hline Cyanidin-3-glucoside & & 2.0 & 67.0 & al. (2011) \\
\hline Pelargonidin & & 8.0 & 36.0 & \\
\hline Cyanidin & & 10.0 & 34.0 & \\
\hline Phenolic extracts & & & & \\
\hline Mulberry phenolic extracts & Anthocyanins & 1.6 & 95.1 & Liang et al. (2012) \\
\hline & Phenolics & 39.6 & 38.0 & \\
\hline Rose phenolic extracts & Phenolics & 6.2 & 15.7 & Zhang et al. (2016) \\
\hline Caper phenolic extracts & Chlorogenic acid $(3.3 \mu \mathrm{g} / \mathrm{mL})$ & 5.8 & 33.0 & Siracusa et \\
\hline & 4-Caffeoylquinic acid (1.8 $\mu \mathrm{g} / \mathrm{mL})$ & 1.5 & 26.4 & al. (2011) \\
\hline & 5-Coumaroylquinic acid (0.5 $\mu \mathrm{g} / \mathrm{mL})$ & 3.9 & 25.7 & \\
\hline & 4-Feruloylquinic acid $(0.7 \mu \mathrm{g} / \mathrm{mL})$ & 2.5 & 19.8 & \\
\hline & Rutin $(10.2 \mu \mathrm{g} / \mathrm{mL})$ & 1.7 & total & \\
\hline & Quercetin 3-O-glucoside $(0.2 \mu \mathrm{g} / \mathrm{mL})$ & 3.8 & total & \\
\hline & Kaempferol 3-O-rutinoside $(2.3 \mu \mathrm{g} / \mathrm{mL})$ & 5.0 & total & \\
\hline & Isorhamnetin 3-O-rutinoside $(0.8 \mu \mathrm{g} / \mathrm{mL})$ & 2.3 & total & \\
\hline & Kaempferol 3-O-glucoside $(0.6 \mu \mathrm{g} / \mathrm{mL})$ & 6.6 & total & \\
\hline Sea Fennel phenolic extracts & 3-Caffeoylquinic acid (12.0 g/mL ) & total & total & \\
\hline & Chlorogenic acid (198.6 g/mL ) & 66.0 & 81.7 & \\
\hline & 1-Caffeoylquinic acid (24.3 $\mu \mathrm{g} / \mathrm{mL})$ & 67.4 & 78.1 & \\
\hline & 5-p-Coumaroylquinic acid $(16.6 \mu \mathrm{g} / \mathrm{mL})$ & total & total & \\
\hline & 5-Feruloylquinic acid (25.0 g/mL ) & total & total & \\
\hline & 3,4-Dicaffeoylquinic acid $(15.6 \mu \mathrm{g} / \mathrm{mL})$ & total & total & \\
\hline & 3,5-Dicaffeoylquinic acid $(57.1 \mu \mathrm{g} / \mathrm{mL})$ & total & total & \\
\hline & 4,5-Dicaffeoylquinic acid $(29.6 \mu \mathrm{g} / \mathrm{mL})$ & total & total & \\
\hline
\end{tabular}


Table 4. Stability of phenolic compounds during gastrointestinal digestion - (continued)

\begin{tabular}{|c|c|c|c|c|}
\hline \multirow{2}{*}{ Digested material } & & \multicolumn{2}{|c|}{ Phenolics loss (\%) } & \multirow{2}{*}{ References } \\
\hline & & Gastric phase & Intestinal phase & \\
\hline \multirow{23}{*}{$\begin{array}{l}\text { Grape pomace } \\
\text { phenolic extracts }\end{array}$} & Galloylshikimic acid (3.4 mg/g) & & total & \multirow[t]{23}{*}{ Corrêa et al. (2017) } \\
\hline & Proanthocyanidine B dimer (25.4 mg/g) & & 91.4 & \\
\hline & Digalloylquinic acid (2.3mg/g) & & 95.2 & \\
\hline & (-)-Epicatechin (7.3 mg/g) & & 76.3 & \\
\hline & (+)-Catechin (7.3 mg/g) & & 75.7 & \\
\hline & Digalloylshikimic acid (1.9 mg/g) & & Total & \\
\hline & Proanthocyanidine B trimer $(8.5 \mathrm{mg} / \mathrm{g})$ & & 93.4 & \\
\hline & Proanthocyanidine B tretramer $(6.2 \mathrm{mg} / \mathrm{g})$ & & 93.9 & \\
\hline & Myricetin-O-hexoside(1.4 mg/g) & & 19.7 & \\
\hline & Quercetin-3-0-glucuronide(0.6 mg/g) & & Total & \\
\hline & Quercetin-3-O-glucoside(0.5 mg/g) & & 55.8 & \\
\hline & Laricitrin-O-hexoside(0.4 mg/g) & & total & \\
\hline & Quercetin-O-pentoside(0.4 mg/g) & & total & \\
\hline & Quercetin-O-rhamnoside(0.4 mg/g) & & 36.8 & \\
\hline & Isorhamnetin-3-O-glucoside(0.5 mg/g) & & total & \\
\hline & Methylisorhamnetin derivative $(0.3 \mathrm{mg} / \mathrm{g})$ & & total & \\
\hline & $\begin{array}{l}\text { Total non-anthocyanin } \\
\text { compounds }(66.6 \mathrm{mg} / \mathrm{g})\end{array}$ & & 87.5 & \\
\hline & Petunidin-3-0-glucoside (0.6 mg/g) & & 68.9 & \\
\hline & Peonidin-3-O-glucoside (1.6 mg/g) & & 85.4 & \\
\hline & Malvidin-3-O-glucoside (3.4 mg/g) & & 90.0 & \\
\hline & Peonidin-3-O-acetylglucoside $(0.7$ mg/g) & & 74.1 & \\
\hline & Malvidin-3-O-acetylglucoside (0.7 mg/g) & & 73.9 & \\
\hline & Total anthocyanin compounds $(7.0 \mathrm{mg} / \mathrm{g})$ & & 84.0 & \\
\hline \multicolumn{5}{|l|}{ Food matrix } \\
\hline \multirow[t]{13}{*}{ Chokeberry juice concentrates } & Cyanidin 3-galactoside (362 $\mu \mathrm{g} / \mathrm{mL})$ & -3.3 & 39.2 & \multirow{13}{*}{$\begin{array}{l}\text { Bermúdez-Soto } \\
\text { et al. ( } 2007)\end{array}$} \\
\hline & Cyanidin 3-glucoside $(41.6 \mu \mathrm{g} / \mathrm{mL})$ & -3.8 & 43.3 & \\
\hline & Cyanidin 3-arabinoside $(240 \mu \mathrm{g} / \mathrm{mL})$ & -2.3 & 44.9 & \\
\hline & Cyanidin 3-xyloside $(29 \mu \mathrm{g} / \mathrm{mL})$ & -4.1 & 50.8 & \\
\hline & Cyanidin $(7.2 \mu \mathrm{g} / \mathrm{mL})$ & -350 & total & \\
\hline & Quercetin hexoside pentoxide $(22.4 \mu \mathrm{g} / \mathrm{mL})$ & 4.5 & 28.6 & \\
\hline & Quercetin 3-galactoside (27 $\mu \mathrm{g} / \mathrm{mL})$ & 2.2 & 15.5 & \\
\hline & Quercetin 3-glucoside $(37.2 \mu \mathrm{g} / \mathrm{mL})$ & 7.0 & 19.1 & \\
\hline & Quercetin 3-rutinoside $(88.8 \mu \mathrm{g} / \mathrm{mL})$ & 5.9 & 29.6 & \\
\hline & Quercetin $(3.6 \mu \mathrm{g} / \mathrm{mL})$ & -5.6 & Total & \\
\hline & Neochlorogenic acid (318 $\mu \mathrm{g} / \mathrm{mL})$ & 3.8 & 28.0 & \\
\hline & Chlorogenic aid (296 $\mu \mathrm{g} / \mathrm{mL})$ & 3.4 & -23.9 & \\
\hline & Other flavan-3-ols $(710 \mu \mathrm{g} / \mathrm{mL})$ & 14.9 & 19.3 & \\
\hline \multirow[t]{4}{*}{ Strawberry yoghurt } & (+)-Catechin $(534.8 \mu \mathrm{g} / \mathrm{g})$ & 10.9 & 47.0 & \multirow{4}{*}{$\begin{array}{l}\text { Oliveira and } \\
\text { Pintado (2015) }\end{array}$} \\
\hline & Quercetin-3-rutinoside $(11.0 \mu \mathrm{g} / \mathrm{g})$ & 18.2 & 40.0 & \\
\hline & Ellagic acid (8.6 $\mu \mathrm{g} / \mathrm{g})$ & 7.0 & 3.5 & \\
\hline & Cyanidin-3-glucoside $(6.5$ g/g) & -3.1 & 46.2 & \\
\hline
\end{tabular}


Table 4. Stability of phenolic compounds during gastrointestinal digestion - (continued)

\begin{tabular}{llll}
\hline \multirow{2}{*}{ Digested material } & \multicolumn{2}{c}{ Phenolics loss (\%) } \\
\cline { 3 - 4 } & & Gastric phase Intestinal phase \\
\hline & Pelargonidin-3-glucoside $(70.6 \mu \mathrm{g} / \mathrm{g})$ & -11.6 & 65.3 \\
Peach yoghurt & Pelargonidin-3-rutinoside $(6.7 \mu \mathrm{g} / \mathrm{g})$ & -26.7 & 58.2 \\
& (+)-Catechin $(35.5 \mu \mathrm{g} / \mathrm{g})$ & 20.6 & 80.0 \\
& Neochlorogenic acid $(50.4 \mu \mathrm{g} / \mathrm{g})$ & -8.5 & 45.0 \\
& Chlorogenic acid $(46.4 \mu \mathrm{g} / \mathrm{g})$ & -9.9 & 38.6 \\
& Quercetin-3-rutinoside $(7.7 \mu \mathrm{g} / \mathrm{g})$ & -7.8 & 31.2 \\
\hline
\end{tabular}

${ }^{\mathrm{a} O n e}$ stage overall process only.

tracted from cereal bran was found to be efficiently hydrolyzed by these esterases. The known catalytic efficiency is in decreasing order of methyl $p$-coumarate, methyl ferulate, methyl caffeate and methyl sinapate (Andreasen et al., 2001a). However, the same authors reported that ferulic acid was hardly absorbed by the small intestinal epithelial cells by transcellular transport. Owing to the ionization of phenolic acid in the alkalescent intestinal fluid, phenolic acid anions in the small intestine are theoretically less available to passively diffuse through the intestinal mucosa (Andreasen et al., 2001b). Instead, Lafay et al. (2006) found that intact chlorogenic acid molecules could be fast absorbed in rat stomach, proved by infusing phenolics into the ligated stomach of food-deprived rats. Meanwhile, stomach was demonstrated to be an effective site for absorption of phenolic acids, as well as for absorption of quercetin, daidzein, and anthocyanins, even if neither quercetin 3-O-glucoside nor rutin was absorbed from the stomach (Crespy et al., 2002; Passamonti et al., 2003; Piskula et al., 1999). However, according to Andreasen et al. (2001b), there were no data to support the nonabsorbability of phenolic acid in the intestine by passive diffusion. In contrast, more novel absorption ways of phenolic acids and their anions were raised in addition to free diffusions, such as active transport and paracellular transport. Monocarboxylic acid transporters (MCT) are a kind of important active transporter of SLC16 superfamily that could be expressed on the apical membrane of gastric and intestinal epithelial cell, as well as various other tissue cells. They play a major role in cell metabolism and metabolic communication between tissues (Dhananjay et al., 2013). MCT prefer to favor molecules with one carboxylate group to penetrate through the plasma membrane, thus possess a high affinity with phenolic acids and corresponding anions especially those with relatively lower polarity. Konishi et al. $(2004,2006)$ investigated the gastric absorption based on MCT of several phenolic acids which is in the order of gallic acid $=$ chlorogenic acid $<$ caffeic acid $<p$-coumaric acid $=$ ferulic acid. Surprisingly, besides phenolic acids, quercetin and ECG (epicatechin gallate) could also penetrate the apical membrane through MCT (Contreras et al., 2016; Walle, 2004). MCT 1 is the best-known member of the MCT family and has been verified as a transporter of phenolic acids in the intestinal mucosa. It is expressed on both sides of the intestinal cell and transports phenolics especially phenolic acids from the lumen and extracellular fluid into enterocytes (Ziegler et al., 2016). Watanabe et al. (2006) reported that MCT 1 was involved in the uptake of salicylic acid but not $p$-coumaric acid and fluorescein, thus they doubted the phenolics transportability of MCT 1 . Meanwhile, some contradictory reports about localization (only apical or only basal membrane) of MCT 1 were compared in this study. Apart from MCT, sodiumcoupled monocarboxylate transporter 1 (SMCT1) has also been suggested as the efflux transporter of phenolic acids, which is a kind of basolateral sodium/potassium pumps of enterocytes also actively transporting various short chain fatty acids into endothelial cells, it is mainly expressed in the intestine rather than the stomach (Ziegler et al., 2016). Meanwhile, paracellular transport was proposed to serve as the absorption mechanism of the phenolic acids (Anderson, 2001; Domínguez-Avila et al., 2017; Konishi et al., 2003). Different from all the transcellular transport ways described above, the paracellular pathway happens on intercellular tight junctions which is a narrow gap that separates the neighboring enterocytes by certain proteins called claudins (Anderson, 2001). The low selectivity of these proteins (resistance varies by 100,000 -fold between "tight" and "leaky" epithelia) allows not only susceptible passing of hydrophobic and neutral molecules, but also the permeation of hydrophilic molecules that are unable to permeate through the lipid membrane by the transcellular pathway of absorption (Anderson, 2001). It provides the theoretical support why small-mass phenolics that are charged, such as ferulic, chlorogenic, gallic and rosmarinic acids, were observed in vivo before they enter into the colon (Konishi et al., 2004a; Konishi et al., 2005; Konishi et al., 2006). The colonic metabolite of phenolic acids, 3,4-dihydroxyphenylpropionic acid, was found to be absorbed via paracellular transport as well as via MCT transporters. However, compared with that of phenolics transported by both transcellular and paracellular ways, the absorbability of phenolics only transported through paracellular is much lower, namely a quite low absorbability for phenolic acids by paracellular pathway (Konishi et al., 2004b, 2005; Lafay and Gil-Izquierdo, 2008).

These aforementioned phenolic transporters are expressed not only in the digestive tract but also other blood-tissue barriers shown in Table 6. Meanwhile, some transporters cannot be found in the gastrointestinal cell but present in other tissues such as renal medulla which is involved in the intake of phenolics and corresponding metabolites from extracellular fluid and urine. They are glucose transporters (GLUT 1 and GLUT 4), organic anion transporters (bilitranslocase, OAT 1, OAT 3 and OAT 4), and organic anion transporting polypeptides (OATP1A2 and OATP2B1). GLUT 1 could be found in erythrocytes, brain, placenta, adipose cell, and muscle, and GLUT 4 is extensively distributed in the brain, muscle, heart, and adipose cell and is insulin sensitive; their transportability of phenolics depends on substrate concentration gradient (Wood and Trayhurn, 2003). Bilitranslocase is a kind of bilirubin active transporter found on the liver plasma membrane and is also broadly distributed in other epithelium or endothelium barriers of the kidney, digestive tract, blood vessel, and brain. It is found to have a high affinity for various dietary flavonoids especially the glycoside-type. In a previous report, a total of 17 anthocyanidins and their mono- and diglycosides presented interaction effect on transport site of bilitranslocase which prefer to capture hy- 
Table 5. In vitro absorption evaluation by dialysis

\begin{tabular}{|c|c|c|c|c|}
\hline Sample & & $\begin{array}{l}\text { Dialyzed } \\
\text { rate (\%) }\end{array}$ & Absorption medium & Method Source \\
\hline \multirow[t]{2}{*}{ Apple } & Phenolics & 55 & \multirow[t]{2}{*}{10 kDa dialysis membrane } & \multirow[t]{2}{*}{ Bouayed et al. (2011) } \\
\hline & Flavonoids & 38 & & \\
\hline \multirow[t]{4}{*}{ Orange juice } & Flavanone & $12-36$ & \multirow[t]{4}{*}{12 kDa dialysis membrane } & \multirow{4}{*}{$\begin{array}{l}\text { Gil-Izquierdo et } \\
\text { al. (2001) }\end{array}$} \\
\hline & Narirutin & $11-31$ & & \\
\hline & Hesperidin & $16-37$ & & \\
\hline & Vicenin-2 & $19-30$ & & \\
\hline \multirow[t]{4}{*}{ Orange juice } & Flavanone & $12-20$ & \multirow[t]{18}{*}{12 kDa dialysis membrane } & \multirow{18}{*}{$\begin{array}{l}\text { Gil-Izquierdo et } \\
\text { al. (2002) }\end{array}$} \\
\hline & Narirutin & $12-21$ & & \\
\hline & Hesperetin & $12-21$ & & \\
\hline & Vicenin-2 & $13-22$ & & \\
\hline \multirow[t]{7}{*}{ Strawberry } & Cyaniding-3-glucoside & $0-6$ & & \\
\hline & Pelargonidin-3-glucoside & $1-13$ & & \\
\hline & Pelargonidin rutinoside & $1-12$ & & \\
\hline & Ellagic acid arabinoside & $5-21$ & & \\
\hline & Ellagic acid & $6-173$ & & \\
\hline & Quercetin-3-glucoside & $4-28$ & & \\
\hline & Kaemferol-3-glucoside & $19-27$ & & \\
\hline \multirow[t]{7}{*}{ Strawberry jam } & Cyaniding-3-glucoside & $0-2$ & & \\
\hline & Pelargonidin-3-glucoside & $1-4$ & & \\
\hline & Pelargonidin rutinoside & $1-4$ & & \\
\hline & Ellagic acid arabinoside & 6 & & \\
\hline & Ellagic acid & $6-10$ & & \\
\hline & Quercetin-3-glucoside & $5-6$ & & \\
\hline & Kaemferol-3-glucoside & $12-27$ & & \\
\hline \multirow[t]{3}{*}{ White or Whole-meal Bread } & Ferulic acid & $61-77$ & \multirow[t]{3}{*}{ Unknown dialysis membrane } & \multirow[t]{3}{*}{ Anson et al. (2009) } \\
\hline & $p$-Coumaric acid & $63-78$ & & \\
\hline & Sinapic acid & 89-92 & & \\
\hline \multirow[t]{3}{*}{ Whole-meal Bread } & Ferulic acid & $2.5-5.1$ & \multirow[t]{3}{*}{ 5-8 kDa dialysis membrane } & \multirow[t]{3}{*}{ Hemery et al. (2010) } \\
\hline & $p$-Coumaric acid & $5.9-15$ & & \\
\hline & Sinapic acid & $20-60$ & & \\
\hline \multirow[t]{12}{*}{ Soymilk } & Flavonoids & 15 & \multirow[t]{12}{*}{12 kDa dialysis membrane } & \multirow{12}{*}{$\begin{array}{l}\text { Rodríguez-Roque } \\
\text { et al. (2013a) }\end{array}$} \\
\hline & Phenolics & 20 & & \\
\hline & Hesperidin & 14 & & \\
\hline & Naringenin & 21 & & \\
\hline & Quercetin & 17 & & \\
\hline & Catechin & 28 & & \\
\hline & Rutin & \multirow{6}{*}{0} & & \\
\hline & Gallic acid & & & \\
\hline & p-Hydroxybenzoic acid & & & \\
\hline & $p$-Coumaric acid & & & \\
\hline & Ferulic acid & & & \\
\hline & Sinapic acid & & & \\
\hline
\end{tabular}


Table 5. In vitro absorption evaluation by dialysis - (continued)

\begin{tabular}{|c|c|c|c|c|}
\hline Sample & & $\begin{array}{l}\text { Dialyzed } \\
\text { rate }(\%)\end{array}$ & Absorption medium & Method Source \\
\hline \multirow[t]{11}{*}{ Mixed fruit juice } & Phenolics & 12 & \multirow[t]{11}{*}{12 kDa dialysis membrane } & \multirow{11}{*}{$\begin{array}{l}\text { Rodríguez-Roque } \\
\text { et al. ( } 2013 b)\end{array}$} \\
\hline & Caffeic acid & 0 & & \\
\hline & Chlorogenic acid & 11 & & \\
\hline & $p$-Coumaric acid & 17 & & \\
\hline & Ferulic acid & 26 & & \\
\hline & Sinapic acid & 18 & & \\
\hline & Hesperidin & 18 & & \\
\hline & Naringenin & 19 & & \\
\hline & Rutin & 22 & & \\
\hline & Quercetin & 29 & & \\
\hline & Catechin & 23 & & \\
\hline \multirow[t]{5}{*}{ Durum Wheat Bran } & Ferulic acid & 32 & \multirow[t]{5}{*}{12 kDa dialysis membrane } & \multirow[t]{5}{*}{ Zaupa et al. (2014) } \\
\hline & $p$-Coumaric acid & 100 & & \\
\hline & Sinapic acid & 79 & & \\
\hline & Caffeic acid & 52 & & \\
\hline & p-Hydroxybenzoic acid & 98 & & \\
\hline \multirow[t]{2}{*}{ Cooked finger Millet } & Phenolics & $16-37$ & \multirow[t]{4}{*}{10 kDa dialysis membrane } & \multirow{4}{*}{$\begin{array}{l}\text { Hithamani and } \\
\text { Srinivasan (2014) }\end{array}$} \\
\hline & Flavonoids & $15-50$ & & \\
\hline \multirow[t]{2}{*}{ Cooked pearl Millet } & Phenolics & $73-96$ & & \\
\hline & Flavonoids & $6-52$ & & \\
\hline \multirow[t]{2}{*}{ Raspberry } & Phenolics & 10 & \multirow[t]{2}{*}{$12 \mathrm{kDa}$ dialysis membrane } & \multirow{2}{*}{$\begin{array}{l}\text { McDougall et } \\
\text { al. (2005) }\end{array}$} \\
\hline & Anthocyanins & 5 & & \\
\hline \multirow[t]{19}{*}{ Maqui berry } & Rutin & 2.2 & \multirow{19}{*}{$\begin{array}{l}12-14 \text { kDa dialysis } \\
\text { membrane }\end{array}$} & \multirow{19}{*}{$\begin{array}{l}\text { Lucas-Gonzalez } \\
\text { et al. (2016) }\end{array}$} \\
\hline & Ellagic acid & 0.3 & & \\
\hline & Quercetin-3-O-galactoside & 4.9 & & \\
\hline & Dimethoxy-quercetin & 0.04 & & \\
\hline & $\begin{array}{l}\text { Delphinidin-3-sambubioside- } \\
\text { 5-glucoside }\end{array}$ & & & \\
\hline & Delphinidin-3,5-diglucoside & & & \\
\hline & Delphinidin-3-glucoside & & & \\
\hline & Cyanidin-3,5-diglucoside & & & \\
\hline & Delphinidin-3-sambubioside & & & \\
\hline & Cyanidin-3-glucoside & & & \\
\hline & Cyanidin-3-sambubioside & & & \\
\hline & Cyanidin-3-sambubioside-5-glucoside & 0 & & \\
\hline & Myricetin-3-galactoside & & & \\
\hline & Myricetin-3-glucoside & & & \\
\hline & Quercetin-galloyl-hexoside & & & \\
\hline & Quercetin-3-glucoside & & & \\
\hline & Quercetin-3-xyloside & & & \\
\hline & Myricetin & & & \\
\hline & Quercetin & & & \\
\hline \multirow[t]{2}{*}{ Mulberry extracts } & Anthocyanins & 0.34 & \multirow[t]{2}{*}{$3.6 \mathrm{kDa}$ dialysis membrane } & \multirow[t]{2}{*}{ Liang et al. (2012) } \\
\hline & Phenolics & 7.33 & & \\
\hline
\end{tabular}




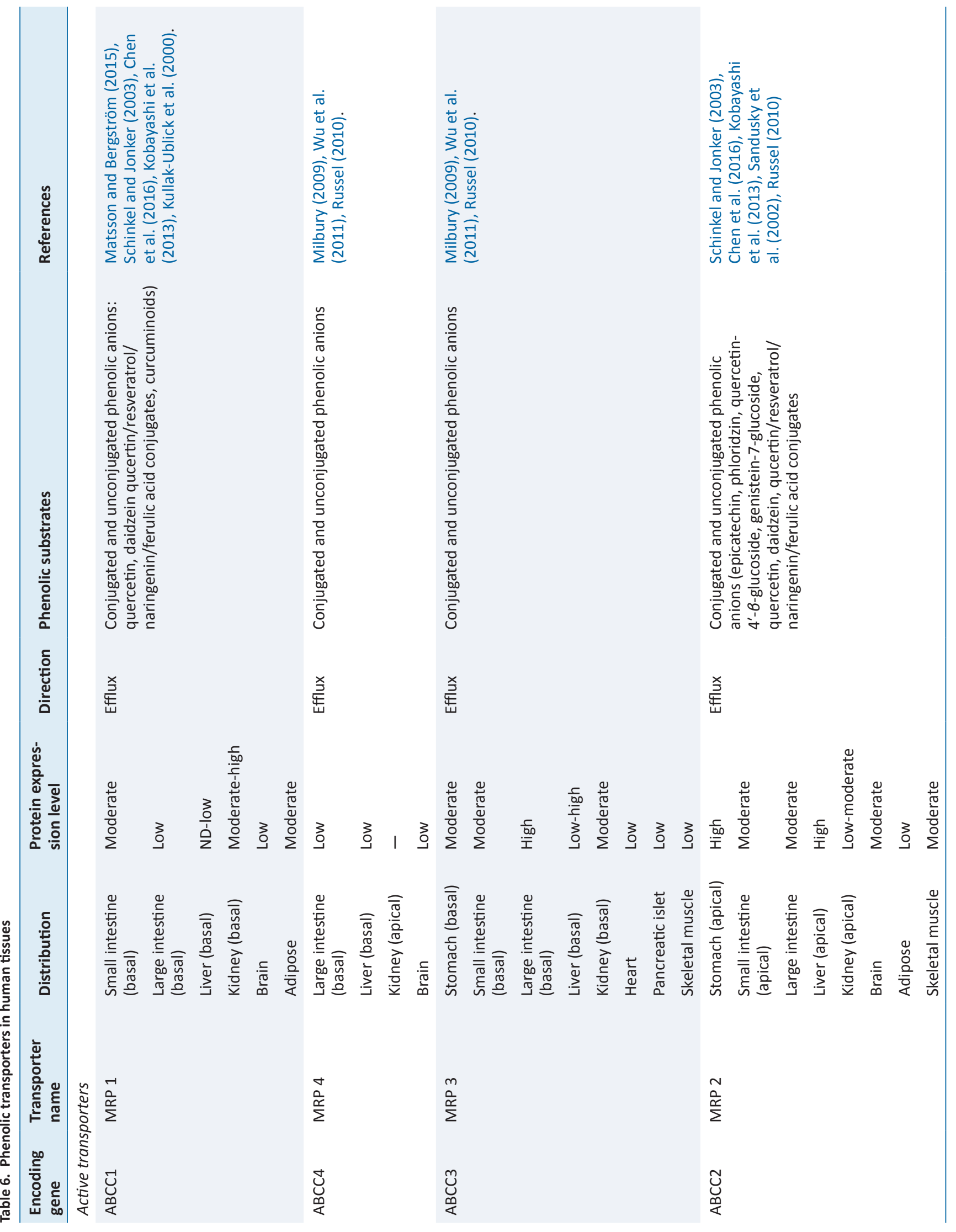




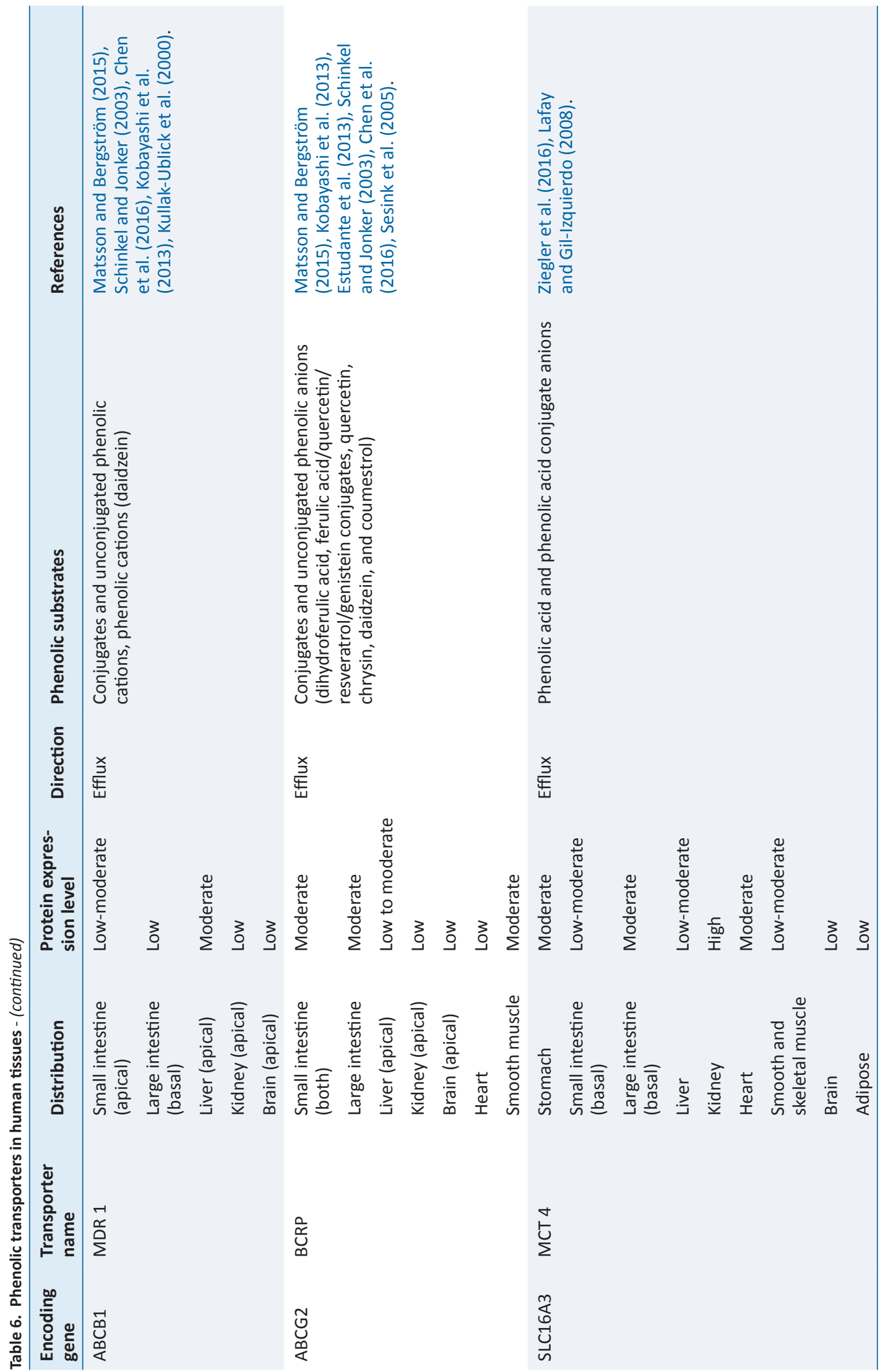




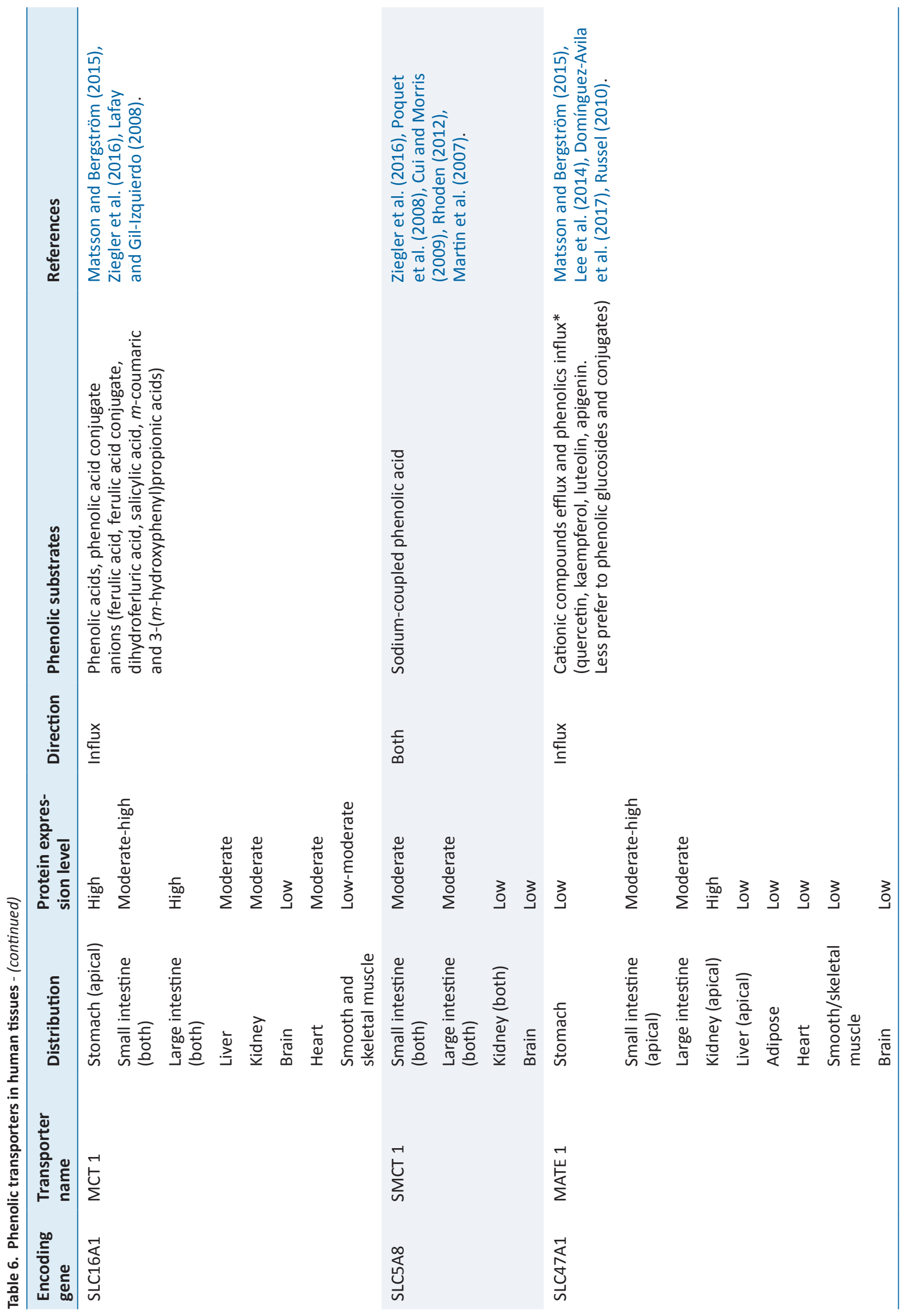




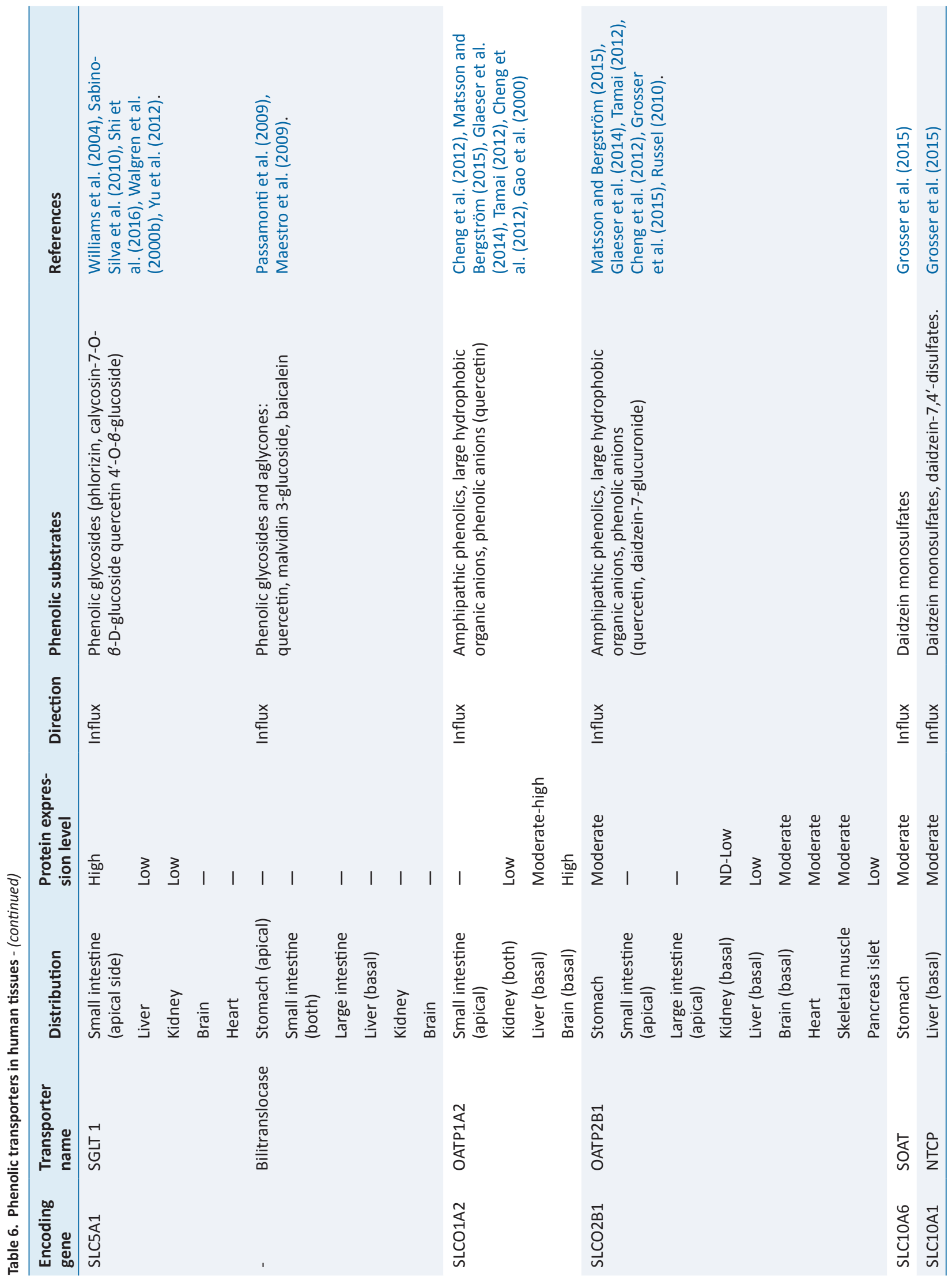




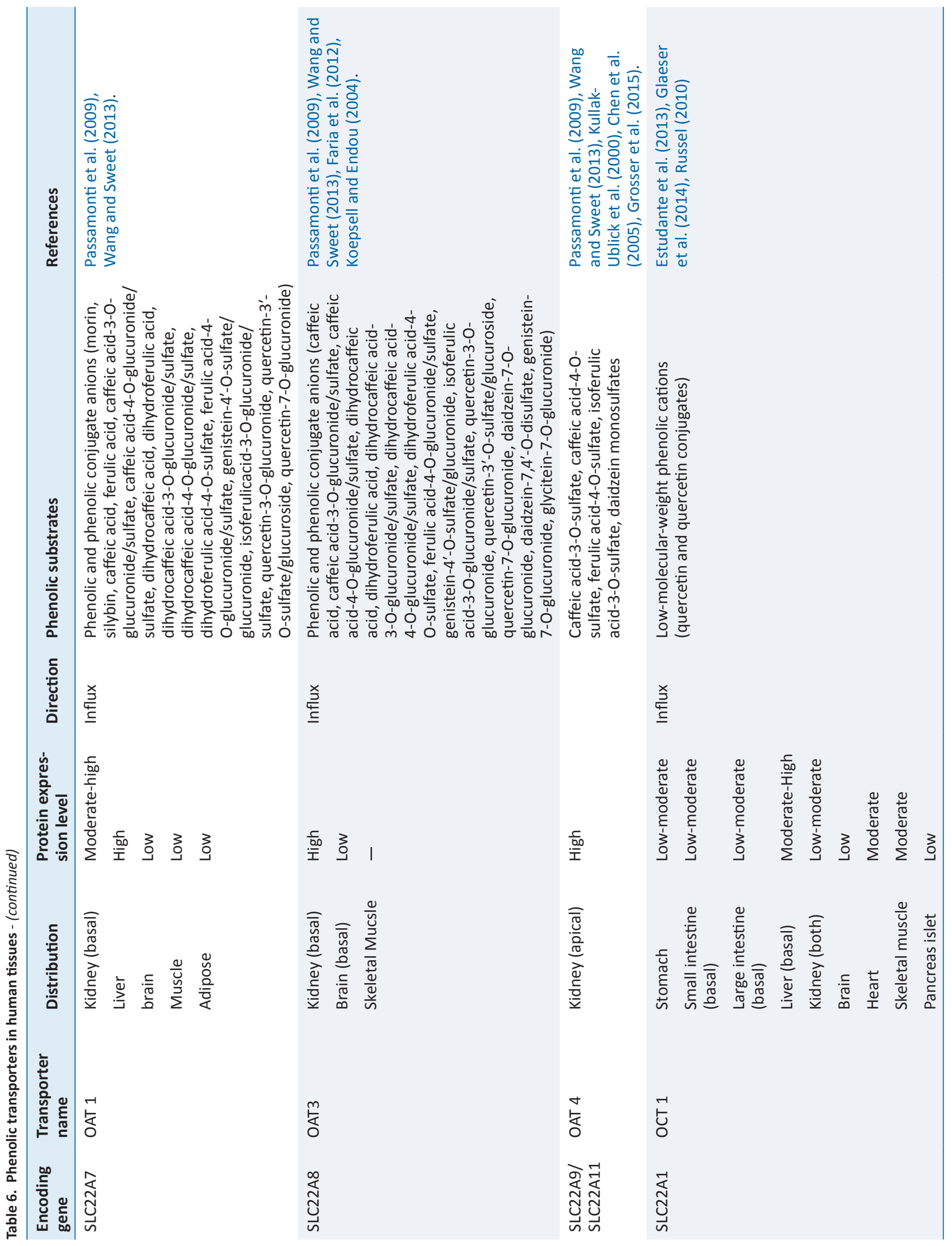




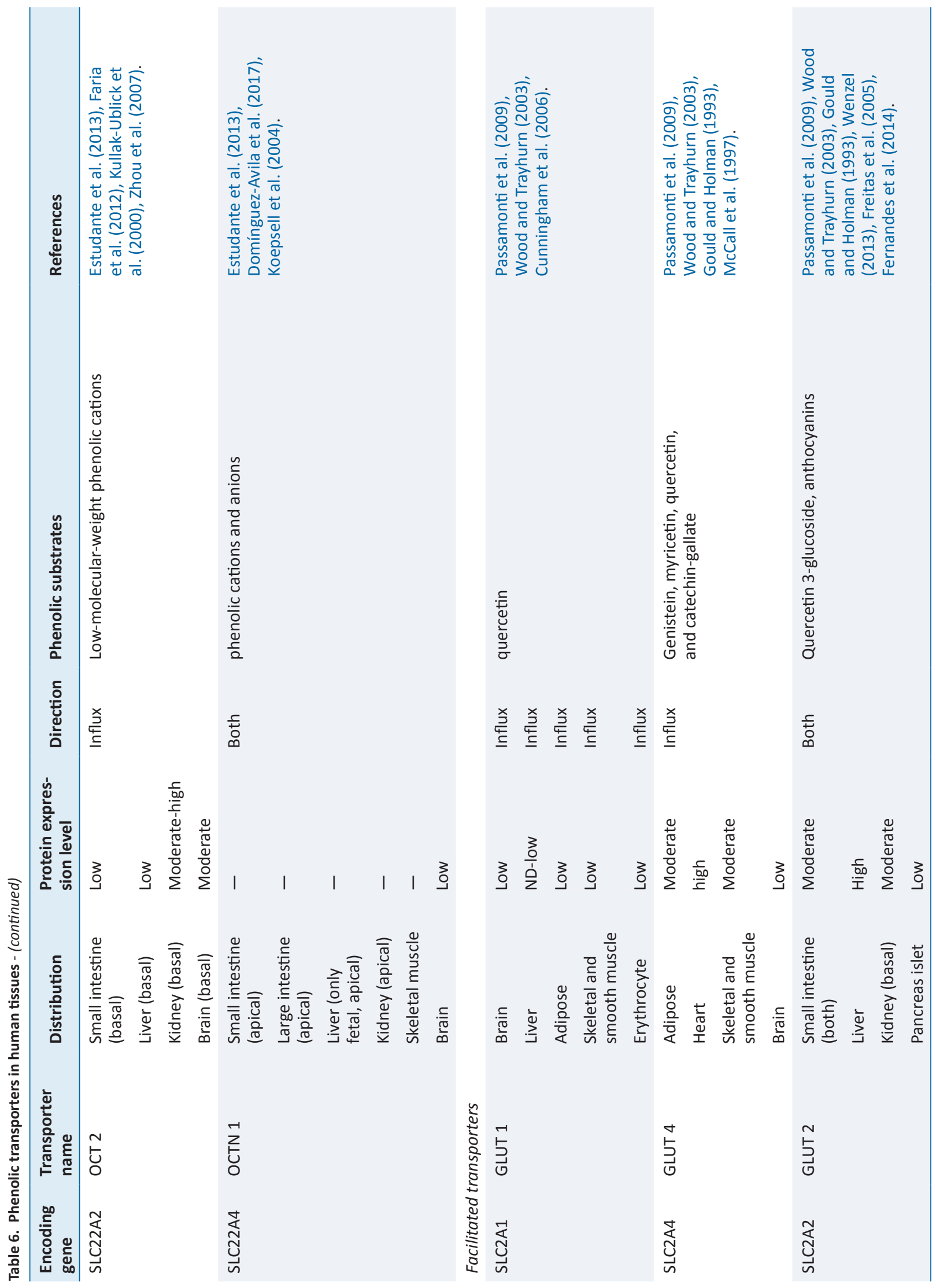




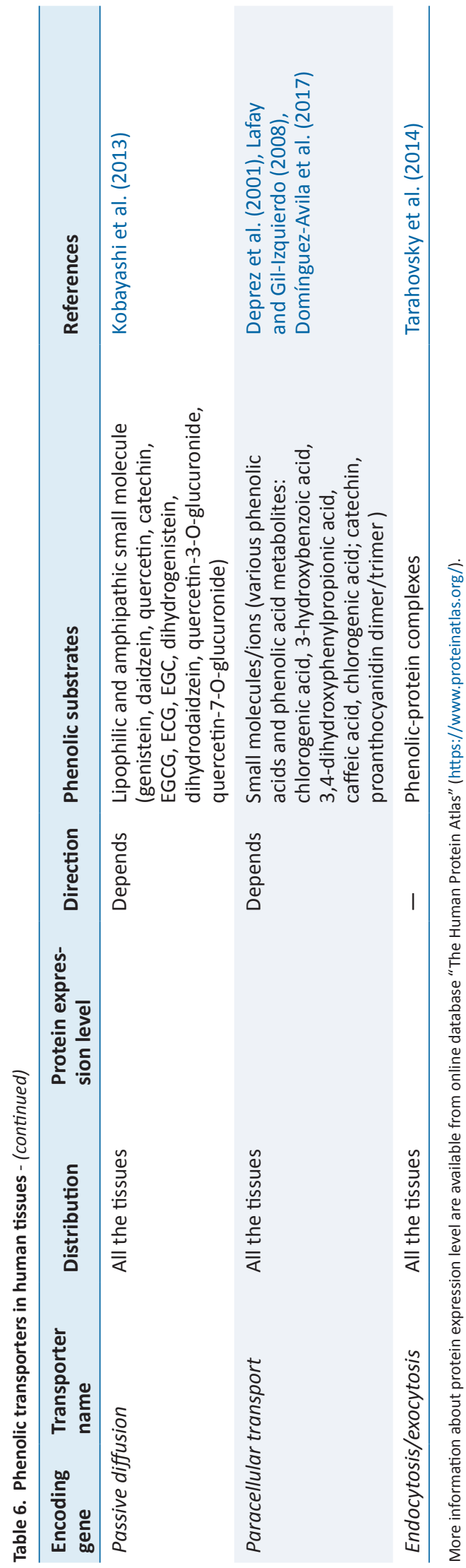

drophilic groups including glycosyl moiety and/or B/A rings of flavonoid glycosides and aglycones, thus deducing that bilitranslocase could play a role in the bioavailability of anthocyanins (Passamonti et al., 2002). Before long, two reports from the same lab proved the absorption ability of bilitranslocase for malvidin-3-O-glucoside and/or quercetin in the rat stomach, as well as in the human vein endothelial and hepatic cell lines (Maestro et al., 2009; Passamonti et al., 2003). Ingestion of flavonoid-rich beverages is acutely causing vasodilation, the bilitranslocase-mediated flavonoids uptake of vascular endothelial cells was regarded as the main cause (Maestro et al., 2009). However, though bilitranslocase is a significant way to absorb anthocyanin at the apical side of stomach and intestine, there is no effective efflux transporters except GLUT 2 (involved with none of MRP 1, MRP 3, MRP 4, SMCT 1, MCT 4, OCTN 1) for them localizing on basal membrane (Fernandes et al., 2014; Passamonti et al., 2009). One cause might be the unavailability of anthocyanins' metabolism in the gastric mucosa. Therefore, much anthocyanins were retained in the epithelium of digestive tract (up to $60 \%$ ) or transported back to the lumen through efflux transporters, rather than to penetrate into the blood; they may partially enter into systemic circulation through interacting with lipoproteins in enterocytes (Passamonti et al., 2009). This provides a potential explanation for the lowest bioavailability of anthocyanins among the 6 popular dietary phenolics mentioned above, namely anthocyanins, isoflavones, flavanones, flavonols, flavanols, and phenolic acids. Human OAT 1 and 3 are highly expressed in the basal membrane of human proximal tubular epithelial cells involved in the uptake of endogenous and exogenous organic anions including phenolics and their metabolites. Whilst OAT 4 positions on the apical side of renal proximal tubule cells and transports phenolics therein as well, which may reabsorb phenolic anions from urine and slow down actual phenolic excretion rate (Hong et al., 2007; Passamonti et al., 2009; Volk, 2014; Wang and Sweet, 2013). OATP1A2 and OATP2B1 belong to organic anion transporting polypeptide family and facilitate the accumulation of anionic xenobiotics including phenolics (quercetin) in various cells relying on the mechanism of proton-coupled transport or hydroxyl ion-exchange transport (Glaeser et al., 2014; Tamai, 2012). Overall, the subgroup of OAT, OATP, OCT, and OCTN are all the members of solute carrier (SLC) 22 gene family playing a major role in the homeostasis of organic ions, mainly function in the kidney (proximal tubule cells) and liver (sinusoidal membrane of hepatocytes). They mediate the uptake of organic cations and anions as the first step of urinary and biliary secretion, respectively (Volk, 2014). More details about distribution, flux direction and expression quantity of different free/active/ facilitated transporters are summarised in Table 6.

Beside free diffusion, facilitated diffusion and active transport, the apical endocytosis also contributes to the phenolics uptake of epithelium such as enterocytes and other cells including macrophages. Initially, phenolics such as kaempferol, galangin, diosmetin, luteolin, taxifolin, catechins interact with immunoglobulins/ albumins/lipoproteins in the digestive, extracellular, or intracellular fluids. Then if the complexes were delivered to the specific site of cells (lipid raft on the external leaflet of the membrane), they could enter the cytoplasm through caveolar-/clathrin-dependent endocytosis. More details on endocytosis/exocytosis transport of phenolics are available elsewhere (Blok et al., 1981; Burton and Smith, 1977; Tarahovsky et al., 2014).

\subsubsection{Efflux transport of enterocytes}

After entering of phenolics into the cytoplasm, some lipophilic phenolics could pass through the basal membrane into blood with- 
Table 7. Phenolic transporters within human blood-brain barrier

\begin{tabular}{|c|c|c|c|}
\hline Transporter name & Location on capillary endothelial cells & Orientation & Citation \\
\hline MRP 1 & Both & $\begin{array}{l}\text { Endothelium to blood, } \\
\text { endothelium to brain }\end{array}$ & Faria et al. (2012), Milbury (2009). \\
\hline MRP 4 & Both & $\begin{array}{l}\text { Endothelium to blood, } \\
\text { endothelium to brain }\end{array}$ & Milbury (2009), Sier (2015). \\
\hline MRP 2 & Both & $\begin{array}{l}\text { Endothelium to blood, } \\
\text { endothelium to brain }\end{array}$ & Faria et al. (2012), Milbury (2009). \\
\hline MDR 1 & Luminal & Endothelium to blood & Faria et al. (2012) \\
\hline BCRP & Luminal & Endothelium to blood & Faria et al. (2012), Cheng et al. (2012). \\
\hline MCT 1 & Both & $\begin{array}{l}\text { Blood to endothelium, } \\
\text { brain to endothelium }\end{array}$ & Faria et al. (2012) \\
\hline MATE 1 & - & - & Geier et al. (2013) \\
\hline SGLT 1 & Abluminal & Brain to endothelium & Faria et al. (2012) \\
\hline OCT 2 & Luminal & Blood to endothelium & Faria et al. (2012) \\
\hline OAT 3 & Abluminal & Brain to endothelium & Faria et al. (2012) \\
\hline OATP1A2 & Luminal & Blood to endothelium & Cheng et al. (2012) \\
\hline OATP2B1 & Both & $\begin{array}{l}\text { Blood to endothelium, } \\
\text { Endothelium to brain }\end{array}$ & Faria et al. (2012), Cheng et al. (2012). \\
\hline GLUT 1 & Both & $\begin{array}{l}\text { Blood to endothelium, } \\
\text { endothelium to brain }\end{array}$ & Faria et al. (2012) \\
\hline GLUT 4 & - & - & McCall et al. (1997) \\
\hline
\end{tabular}

out metabolism such as A/B-type proanthocyanidin (Appeldoorn et al., 2009). While more aglycones and glycosides will be metabolized (or not) to produce phenolic conjugates (e.g., glucuronates and sulfates), and then further flux into blood capillary or digestive tract. The specific metabolism process in the intestinal cells is discussed in the next section, but here we focus only on the transport mechanism. First of all, flux direction of phenolics in the cytoplasm based on the localization of transporters (efflux from the gut lumen or endothelial cells into cells or lumen) is considered. While efflux transporters placed in basal membrane, phenolic metabolites succeed in being absorbed and entering into systemic circulation. Multidrug resistance-associated proteins 1, 3 and 4 (MRP 1, 3 and 4) are three well-known efflux transporters located on the basal membrane and belong to multidrug resistance-associated protein (MRP) family which is a subgroup of ATP-binding cassette trans- porter and facilitates to exclude xenobiotics out of cells (Borst et al., 2000). As the highly expressed absorptive transporters in small and/or large intestinal cell, these efflux transporters prefer to transport intracellular phenolic metabolites (organic anions form) into the circular system and avoids toxicity caused by phenolics accumulation in enterocytes (Borst et al., 2000; Estudante et al., 2013).

Similar to the substrates range of MRP 1 and 3, MRP 2 is also an anion transporter can actively transport phenolic conjugates (e.g. naringenin glucuronides, resveratrol glucuronide and sulfate conjugates) as well as non-conjugated anionic phenolics (e.g. epicatechin) out of the intestinal cells, but the flux direction is from cytoplasm to lumen attributed to its expression on apical membrane of small and large intestine (Estudante et al., 2013). As a result, except for cationic anthocyanins, the actual absorbability of various orally ingested phenolics may be suppressed by efflux mechanism

Table 8. Main enzymes in phase I metabolism

\begin{tabular}{|c|c|c|c|}
\hline Category & Main Enzymes & Common Reactions & Products \\
\hline Oxidation & $\begin{array}{l}\text { Alcohol oxidases; Aldehyde oxidases; } \\
\text { Xanthine oxidases; Monoamine } \\
\text { oxidases; Flavin-containing } \\
\text { monooxygenases; Cytochrome P450s. }\end{array}$ & $\begin{array}{l}\text { Aromatic C-oxidation; Aliphatic } \\
\text { C-oxidation; N- and S-oxidation. } \\
\text { Epoxidation; Dehydrogenation; } \\
\text { N-, O- and S-dealkylations. }\end{array}$ & $\begin{array}{l}\text { Phenols; Alcohols; Ketones; } \\
\text { Aldehydes; Epoxides; } \\
\text { Ketenes; Acids; N- and } \\
\text { S-oxides; Amines; Thiols. }\end{array}$ \\
\hline Hydrolysis & $\begin{array}{l}\text { Carboxylesterases; Peptidases; } \\
\text { Epoxide hydrolases; } \\
\text { Cholinesterases; Paraoxonases. }\end{array}$ & Hydrolysis of esters, amides, epoxides. & Alcohols; Acids; Amines. \\
\hline Reduction & $\begin{array}{l}\text { Alcohol Dehydrogenases; Carbonyl } \\
\text { Reductases; NADPH-quinone } \\
\text { oxidoreductases; NADPH- } \\
\text { cytochrome P450 reductases. }\end{array}$ & $\begin{array}{l}\text { Reduction of azo, nitro groups, carbonyl Groups, } \\
\text { sulfoxides and N-oxides, quinones; Reductive } \\
\text { Cleavage of Heteroaromatic Compounds; Disulfide } \\
\text { reduction and reductive dehalogenation. }\end{array}$ & $\begin{array}{l}\text { Phenols; Alcohols; } \\
\text { Ketones; Sulfides; Acids; } \\
\text { Amines; Thiols. }\end{array}$ \\
\hline
\end{tabular}

Adapted from Timbrell and Marrs (2009), Smart and Hodgson (2018), Shimada et al. (2006), and Hodgson et al. (2001). 


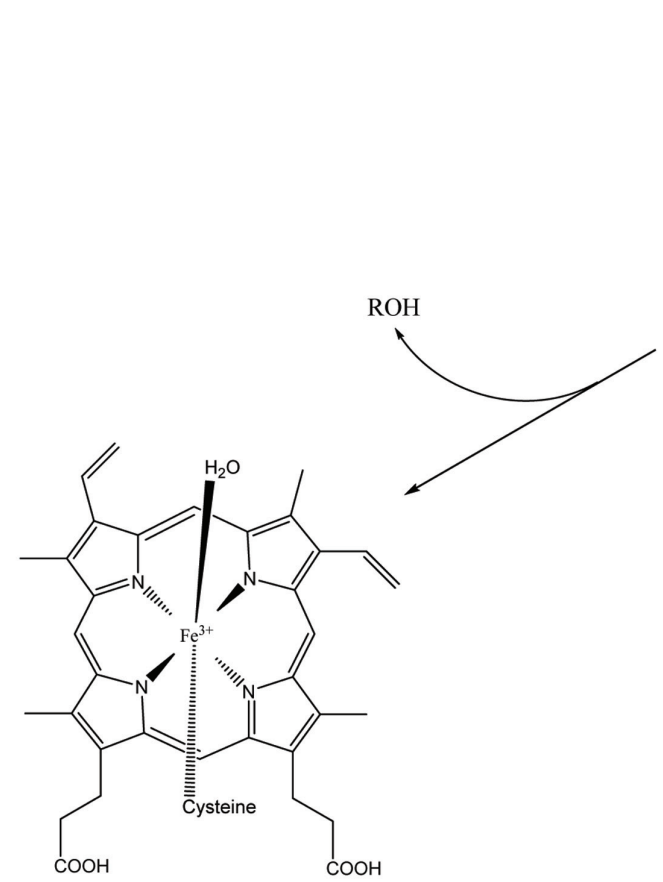

Cytochrome P450s
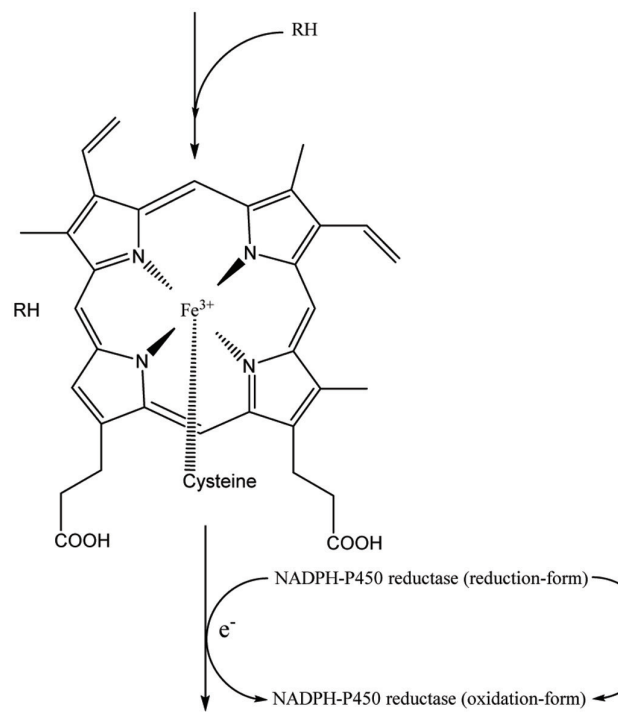

$\mathrm{RH}$
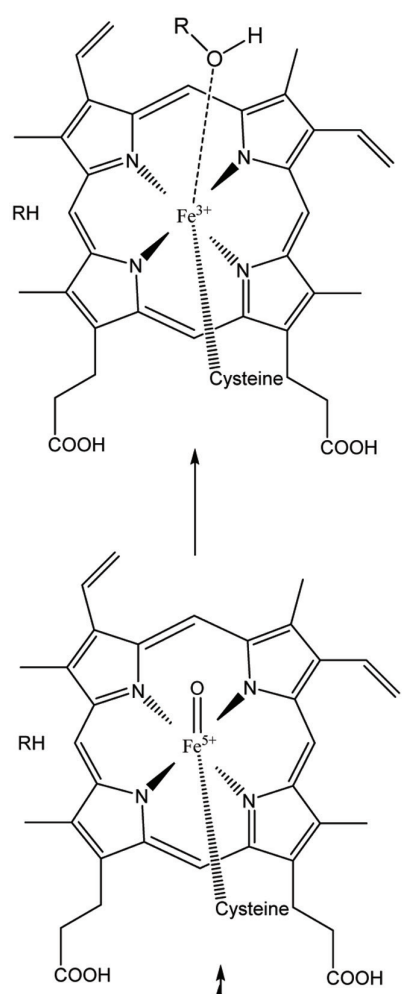

$\mathrm{RH}$
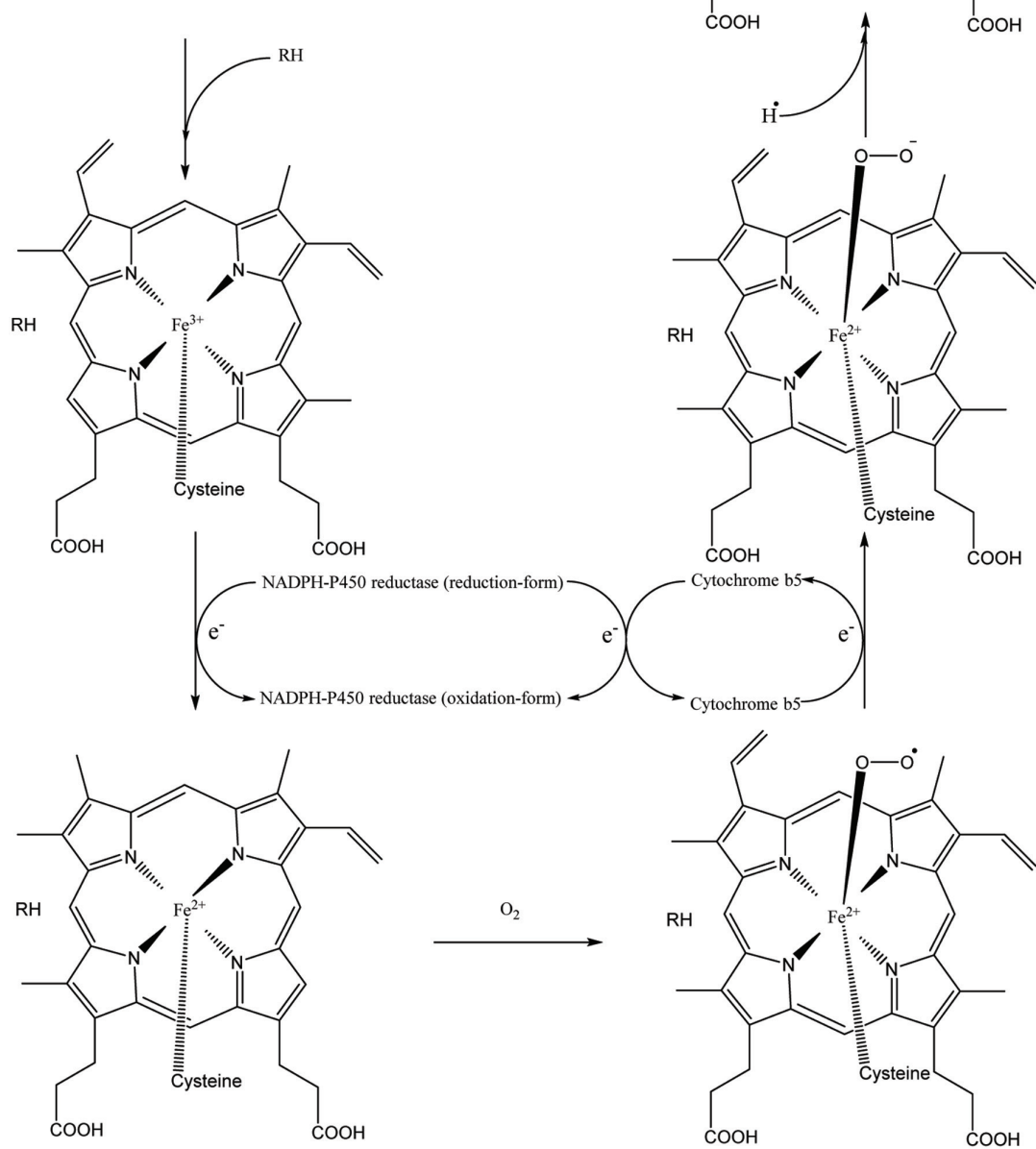

$\mathrm{O}_{2}$

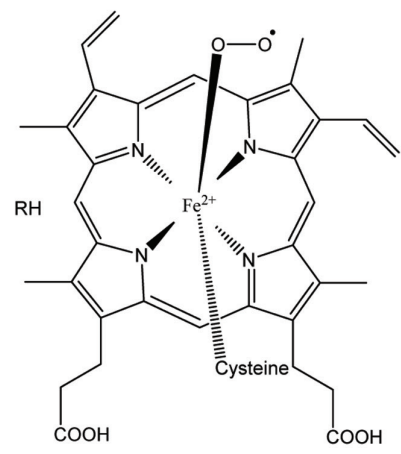

Figure 2. Cytochrome P450 catalytic process. 
Table 9. Products of CYP-450s-catalyzed oxidation

\begin{tabular}{|c|c|c|c|c|}
\hline Substrate & Enzymes & Product(s) & Enzyme source & References \\
\hline Galangin & CYP2C9*, CYP1A1,CYP1A2 & Kaempferol & Human liver microsomes & \multirow{2}{*}{$\begin{array}{l}\text { Otake and } \\
\text { Walle (2002b) }\end{array}$} \\
\hline Kaempferide & CYP1A2*, CYP1A1, CYP2C9 & Kaempferol & Human liver microsomes & \\
\hline Kaempferol & CYP1A1 & Quercetin & $\begin{array}{l}\text { Hamster high-expressed } \\
\text { CYP-450s ovary cell }\end{array}$ & Silva et al. (1997) \\
\hline Chrysin & CYP1A1, CYP1A2 & $\begin{array}{l}\text { Apigenin (major), scutellarein } \\
\text { and isoscutellarein }\end{array}$ & $\begin{array}{l}\text { Aroclor } 1254 \text {-induced } \\
\text { rat liver microsomes }\end{array}$ & \multirow{5}{*}{$\begin{array}{l}\text { Nielsen et al. } \\
\text { (1998), Gradolatto } \\
\text { et al. (2004). }\end{array}$} \\
\hline Naringenin & CYP1A & Eriodictyol & $\begin{array}{l}\text { Aroclor } 1255 \text {-induced } \\
\text { rat liver microsomes }\end{array}$ & \\
\hline Hesperetin & CYP1A & Eriodictyol & $\begin{array}{l}\text { Aroclor } 1256 \text {-induced } \\
\text { rat liver microsomes }\end{array}$ & \\
\hline Apigenin & CYP1A & Luteolin & $\begin{array}{l}\text { Aroclor } 1257 \text {-induced } \\
\text { rat liver microsomes }\end{array}$ & \\
\hline Tamarixetin & CYP1A & Quercetin & $\begin{array}{l}\text { Aroclor } 1258 \text {-induced } \\
\text { rat liver microsomes }\end{array}$ & \\
\hline$\alpha$-Naphthoflavone & CYP-450c, epoxide hydrolase & $\begin{array}{l}\text { 7,8-Dihydro-7,8-dihydroxy- } \\
\alpha \text {-naphthoflavone } \\
\text { 5,6-Dihydro-5,6-dihydroxy- } \\
\alpha \text {-naphthoflavone } \\
\text { 5,6-Oxide- } \alpha \text {-naphthoflavone } \\
\text { 6-Hydroxy- } \alpha \text {-naphthoflavone } \\
\text { 9-Hydroxy- } \alpha \text {-naphthoflavone }\end{array}$ & Rat liver microsomes. & $\begin{array}{l}\text { Andries et al. } \\
\text { (1990), Vyas } \\
\text { et al. (1983). }\end{array}$ \\
\hline Tangeretin & CYP1A & $\begin{array}{l}\text { Demethyl tangeretin or } \\
\text { multi-demethyl tangeretin }\end{array}$ & $\begin{array}{l}\text { Rat and human liver } \\
\text { microsomes. }\end{array}$ & $\begin{array}{l}\text { Canivenc-Lavier } \\
\text { et al. (1993) }\end{array}$ \\
\hline B-Naphthoflavone & Unknown CYP-450s & 8-Hydroxy- $\beta$-naphthoflavone & Rat liver microsomes. & Vyas et al. (1983) \\
\hline B-Naphthoflavone & CYP-450c, epoxide hydrolase & $\begin{array}{l}\text { Trans-7,8-dihydro-7,8- } \\
\text { dihydroxy- } \beta \text {-naphthoflavone } \\
\text { Trans-5,6-dihydro-5,6- } \\
\text { dihydroxy- } \beta \text {-naphthoflavone } \\
\text { 5-Hydroxy- } \beta \text {-naphthoflavone }\end{array}$ & Rat liver microsomes. & \\
\hline
\end{tabular}

of multidrug resistance-associated protein 2 (MRP 2) (Walgren et al., 2000a; Walle, 2004). Besides, several other transporters such as multidrug resistance 1 (MDR 1) and breast cancer resistance protein (BCRP) present an overlapping function and even boarder substrate specificity including hydrophobic flavonoids and other cationic or amphipathic xenobiotics, which contributes to the excretion of these substrates in the renal tubular luminal membrane (Estudante et al., 2013; Volk, 2014). By the way, O'Leary et al. (2003) found that MDR 1 was not the main efflux transporter for quercetin conjugates but MRP 2 was proven to be the one. While BCRP is highly overlapping in substrate specificities with MRP2 and MDR 1 and prone to exclude anionic compounds such as phenolic conjugates (e.g., quercetin, naringenin, resveratrol conjugates) as well as non-conjugated phenolics (e.g., quercetin, genistein, daidzein, and coumestrol). Furthermore, MCT 4 was most recently proposed to be an efflux transporter positioning on the basal side of enterocytes and exclude phenolic acids in the same direction of MRP 1, they facilitate the entrance of phenolics into the blood (Ziegler et al., 2016). These transporters are moderately or highly expressed at the apical membrane of enterocytes thereby limiting the intestinal absorption of many important xenobiotics including dietary phenolics (Estudante et al., 2013). As an example, the relatively lipid-soluble chrysin was estimated to have an actual bioavailability of only $0.003-0.02 \%$ in the normal human body, which should have a better absorbability due to its high membrane permeability; a similar result was also found in rats. Considering the previous data of Walle et al. (1999; 2001) in Caco2 study of chrysin absorption, it may be concluded that efflux of metabolites back into the lumen is one of the important causes for extremely low oral bioavailability of chrysin.

So far, in addition of passive diffusion, transcellular transport, and Endocytosis/exocytosis, 25 transporters are known to significantly affect bioavailability including absorption, translocation, and disposal of dietary phenolics which may be responsible for further phenolic bioactivity in specific tissues such as penetration of phenolics into the blood-brain barrier (Table 7).

\section{In vivo Metabolism}

In the human body, phenolics are one class of xenobiotic whose molecular structures and biochemical properties are modified by specialized enzymatic systems and further conjugated with charged species after absorption, which aims to polarize absorbed xenobiotic and enable them to be transported and excreted in regular routine instead of uncontrollably accumulating in tissues or freely diffusing across membranes. These associated intracorporal biotransformation and transportation pathways after absorption of phenolics are 


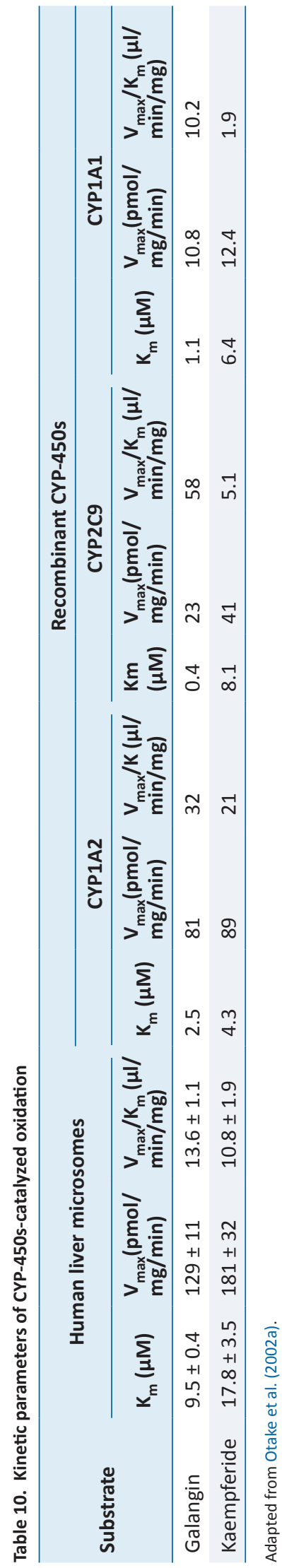

indeed in vivo phenolic metabolism. The classical phenolics metabolism contains three stages: phase I (modification phase), phase II (conjugation phase) and phase III (excretion phase) (Xu et al., 2005). For every phase, the enzyme involved is complex and not entirely understood. Briefly, the phase I metabolism of xenobiotics contains a set of structural modification including thiolation, hydroxylation, amination, N-/O-dealkylation, or carboxylation conducted by certain phase I enzymes (Table 8). For phenolic phase I metabolism, hydroxylation of hydrocarbon sites especially the aromatic carbon catalyzed by a large group of hemoprotein monooxygenases: the cytochromes P450s (CYP-450s) is the most widely studied route (Fig. 2) (De Montellano, 2005; Guengerich, 2007). Recently, cytochrome P450 3A was found to account for the majority of total cytochromes P450 superfamily extensively expressed in the human tissues: liver $(\sim 35 \%)$ and gut $(\sim 82 \%)$, thus acting as the main enzymes involved in the oxidative reaction, followed by CYP2C (one reported $\sim 14 \%$ in liver and $16 \%$ in gut, another one reported $30-40 \%$ in liver) and CYP1A, CYP2A, CYP2D, CYP2E, CYP2J and CYP2D are also abundantly expressed (Domínguez-Avila et al., 2017; Galetin et al., 2010; Heim et al., 2002; Kadlubar and Kadlubar, 2010). As reported earlier, hydroxylation of flavonoids is mainly catalyzed by CYP1A isozymes and demethylation by other CYP-450s (except CYP1A and CYP3A) subgroup (e.g., CYP2C) (Table 9). However, some exceptions such as chrysin, eriodictyol, taxifolin, luteolin, quercetin, myricetin, fisetin, morin or isorhamnetin exist that cannot be found to be oxidized by the normal CYP-450s system (Nielsen et al., 1998; Otake and Walle, 2002b; Silva et al., 1997). The oxidation catalyzed by CYP-450s prefers to happen on both A-ring (hydrogenation of C-5, 6 or C-7, 8; hydroxylation of C-5, 6 or 7, 8; epoxidation of C-5, 6 ) and B-ring (hydroxylation and demethylation of C-3' and/or $4^{\prime}$ until form a 3',4'-dihydroxyl structure) (Andries et al., 1990). For example, hydroxylation of apigenin dominantly happens on C-3', followed by C-5 and 7 positions (Table 9) (Gradolatto et al., 2004). This hydroxylation process may enhance the antioxidant activity of phenolics, and the enhancing extent depends on the hydroxylation ability of the site and the degree of hydroxylation. Simultaneously, the abundance of hydroxyl groups $(\geq 2)$ on the B-ring may prevent further hydroxylation of flavonoids such as quercetin for which no phase I hydroxylated metabolites were detected. Besides, some other factors may affect the phase I metabolism carried by CYP-450s. Polarity dominates the metabolism rate of hydroxylation, the hydrophobic one (with a less free hydroxyl group) was metabolized faster (Nielsen et al., 1998). Demethylation selectively occurs in the B-ring in CYP-1A-mediated monooxygenase system when the methoxy group is positioned at C-4' but not at the C- $3^{\prime}$ (more stable), whereas C-ring structure takes minor and no effect on hydroxylation and demethylation, respectively (Nielsen et al., 1998). On the other side, hydroxyl group on B-ring could be methylated by catechol-O-methyltransferase (COMT) in phase II metabolism, thus it may form a circulation between 4'-O-methylation and 4'-O-demethylation until: 1) transforming to be 3'-O-methyl on catechol group and exclude out of cell; 2) sulfation/glucuronidation of 4'-O-methyl aglycones and produce methylquercetin sulfates/ glucuronides and excluded out of cell; 3 ) directly excluded out of cell (Nielsen et al., 1998; O'Leary et al., 2003). Overall, CYP-450s involved in the metabolism of flavonoids exhibit stereo-selectivity toward the flavonoid substrates.

Apart from the methylation mentioned above, there are several other phase II metabolic reactions such as glucuronidation, sulfation, acetylation, amino acid conjugation, and glutathione conjugation. Amongst them, glucuronidation and sulfation are prevalently found in phenolic metabolism catalyzed by uridine 5'-diphosphoglucuronosyltransferases (UDP-glucuronosyltransferases or UGTs) 
Table 11. Kinetic parameters of glucuronidation and sulfation of galangin

\begin{tabular}{|c|c|c|c|c|c|c|c|}
\hline & \multirow{2}{*}{ Enzyme Source } & \multicolumn{2}{|c|}{$K_{m}(\mu M)$} & \multicolumn{2}{|c|}{$\mathrm{V}_{\max }(\mathrm{pmol} / \mathrm{mg}$ of protein/min) } & \multicolumn{2}{|c|}{$\mathrm{V}_{\max } / \mathrm{K}_{\mathrm{m}}(\mu \mathrm{l} / \mathrm{min} / \mathrm{mg})$} \\
\hline & & Peak 1 & Peak 2 & Peak 1 & Peak 2 & Peak 1 & Peak 2 \\
\hline \multirow[t]{4}{*}{ Recombinant UGTs } & Human liver microsomes & $3.6 \pm 0.7$ & $221 \pm 31$ & $1,521 \pm 252$ & $34,333 \pm 2,167$ & $427 \pm 26$ & $155 \pm 30$ \\
\hline & UGT1A9 & 1.1 & 31.8 & 721 & 3,594 & 655 & 113 \\
\hline & UGT1A1 & N.D. & 6.3 & N.D. & 388 & N.D. & 62.1 \\
\hline & UGT2B15 & N.D. & 15.7 & N.D. & 538 & N.D. & 34.3 \\
\hline \multirow[t]{3}{*}{ Recombinant SULTS } & SULT1A1 & \multicolumn{2}{|c|}{0.21} & \multicolumn{2}{|r|}{3,270} & \multicolumn{2}{|r|}{15,572} \\
\hline & SULT1A3 & \multicolumn{2}{|c|}{37.1} & \multicolumn{2}{|r|}{822} & \multicolumn{2}{|r|}{22.2} \\
\hline & SULT1E1 & \multicolumn{2}{|c|}{1.13} & \multicolumn{2}{|r|}{948} & \multicolumn{2}{|r|}{839} \\
\hline
\end{tabular}

Adapted from Otake et al. (2002a).

and sulfotransferases (SULTs). The same as that of methylation, the reactive sites on phenolics are hydroxyl groups. The glycine conjugation of hydroxybenzoic acid or benzoic acid is also a common phase II reaction catalyzed by benzoyl CoA which finally produces the hippuric acid or hydroxyhippuric acid (Toromanović et al., 2008). Several phase II phenolic metabolites are shown in Table 9. These conjugations caused by UGTs, SULTs, and COMT may result in the alteration of phenolic bioactive properties; some of these metabolites lose their activities. For example, glucuronidation of isoflavonoids (e.g., daidzein and genistein) exert lower biological activities including estrogen receptor binding capacity and natural killer cell activation ability (Wu et al., 2011). The serum metabolic mixture of fisetin (fisetin sulfates/glucuronides) showed lower ability than native fisetin in the inhibition of hemolysis induced by AAPH (2,2'-azobis (2-amidinopropane hydrochloride)) (Shia et al., 2008). A lower value was also shown by glucuronidates/sulfates of flavonols (e.g., quercetin) in delaying the copper-induced lipoprotein oxidation (Morand et al., 1998). The resveratrol sulfates displayed low antiproliferative/antitumor activity, and its corresponding sulfates/glucuronides partially lost their inhibitory activity on COX-1 and 2 compared to the parent resveratrol (Calamini et al., 2010; Hoshino et al., 2010; Miksits et al., 2009; Rotches-Ribalta et al., 2012a). Furthermore, 3-O-glucuronidation and 3'-O-sulfation of quercetin diminished the $\mathrm{PGE}_{2}$ inhibitory activity of the original quercetin in cell test. The $\mathrm{LTB}_{4}$ inhibiting activity of quercetin was also lost due to the 3'- and 3-conjugation of quercetin (3'-O-methylquercetin and quercetin-3'-O-sulfate, quercetin-3-O-glucuronide and 3'-O-methylquercetin-3-O-glucuronide) (Loke et al., 2008). Whereas some phenolics contrarily obtain higher bioactivity as me- tabolites, the glucuronic conjugates of EGCG such as EGCG-3"-Oglucuronide and EGCG-3'-O-glucuronide increase the DPPH scavenging activity compared to that of the EGCG aglycone (Monagas et al., 2010). Based on these studies, there is no doubt that dietary phenolics have extremely low bioavailability and detoxification metabolism which results in an uncertainty of their physiological effects, however, they could still play a vital role on health. Morand et al. (1998) stated that a diet containing $0.2 \%$ quercetin enhances about $60 \%$ antioxidant ability of rat plasma compared with that of the control group. Other in vivo antioxidant activity tests of healthy human were demonstrated in a more recent review (Martins et al., 2016).

Generally, compared with phase II metabolism, phase I metabolism pathway of phenolics mediated by CYPs is relatively less encountered (may result from the low reaction rate of CYPs; refer to Tables 10-12) (Chen et al., 2014). Phase I metabolism is not such a necessary prerequisite for phase II metabolism of xenobiotics although a contribution from CYP-mediated oxidation cannot be entirely ignored in phenolic metabolism. Phenolics could first carry through phase I and then phase II metabolism or directly proceed to phase II metabolism. Meanwhile, phase III stage of phenolics is the phenolic excretion process which is involved with various efflux transporters in the kidney, liver, and intestine (refer to Table 6) is not described here.

\subsection{Phase I and phase II enzyme distribution}

In the human body, intestine and liver are the two most impor-

Table 12. Kinetic parameters of COMT-catalyzed O-methylation

\begin{tabular}{|c|c|c|c|c|c|}
\hline & \multirow{2}{*}{$\begin{array}{l}\text { Hamster kidney cytosol } \\
\text { Rate (pmol/mg/min) }\end{array}$} & \multicolumn{4}{|c|}{ Recombinant COMT from Porcine liver } \\
\hline & & Rate (pmol/mg/min) & $K_{m}(\mu M)$ & $\mathrm{V}_{\max }(\mathrm{pmol} / \mathrm{mg} / \mathrm{min})$ & $\mathrm{V}_{\max } / \mathrm{K}_{\mathrm{m}}(\mu \mathrm{l} / \mathrm{min} / \mathrm{mg})$ \\
\hline Quercetin & $109 \pm 11$ & $9,100 \pm 47$ & 6.1 & 14,870 & 2,438 \\
\hline Fisetin & $119 \pm 8$ & $13,100 \pm 101$ & 4.8 & 17,700 & 3,687 \\
\hline 2-Hydroxyestradiol & $32 \pm 3$ & $2,206 \pm 21$ & 16.2 & 4,123 & 255 \\
\hline 4-Hydroxyestradiol & $9 \pm 2$ & $414 \pm 11$ & 23.4 & 2,560 & 109 \\
\hline Epinephrine & ND & $16 \pm 2$ & 1,036 & 2,754 & 2.66 \\
\hline Norepinephrine & ND & $9 \pm 2$ & 1,149 & 1,990 & 1.73 \\
\hline Dopamine & ND & $31 \pm 3$ & 1,018 & 5,182 & 5.09 \\
\hline
\end{tabular}

Adapted from Zhu et al. (1994). 


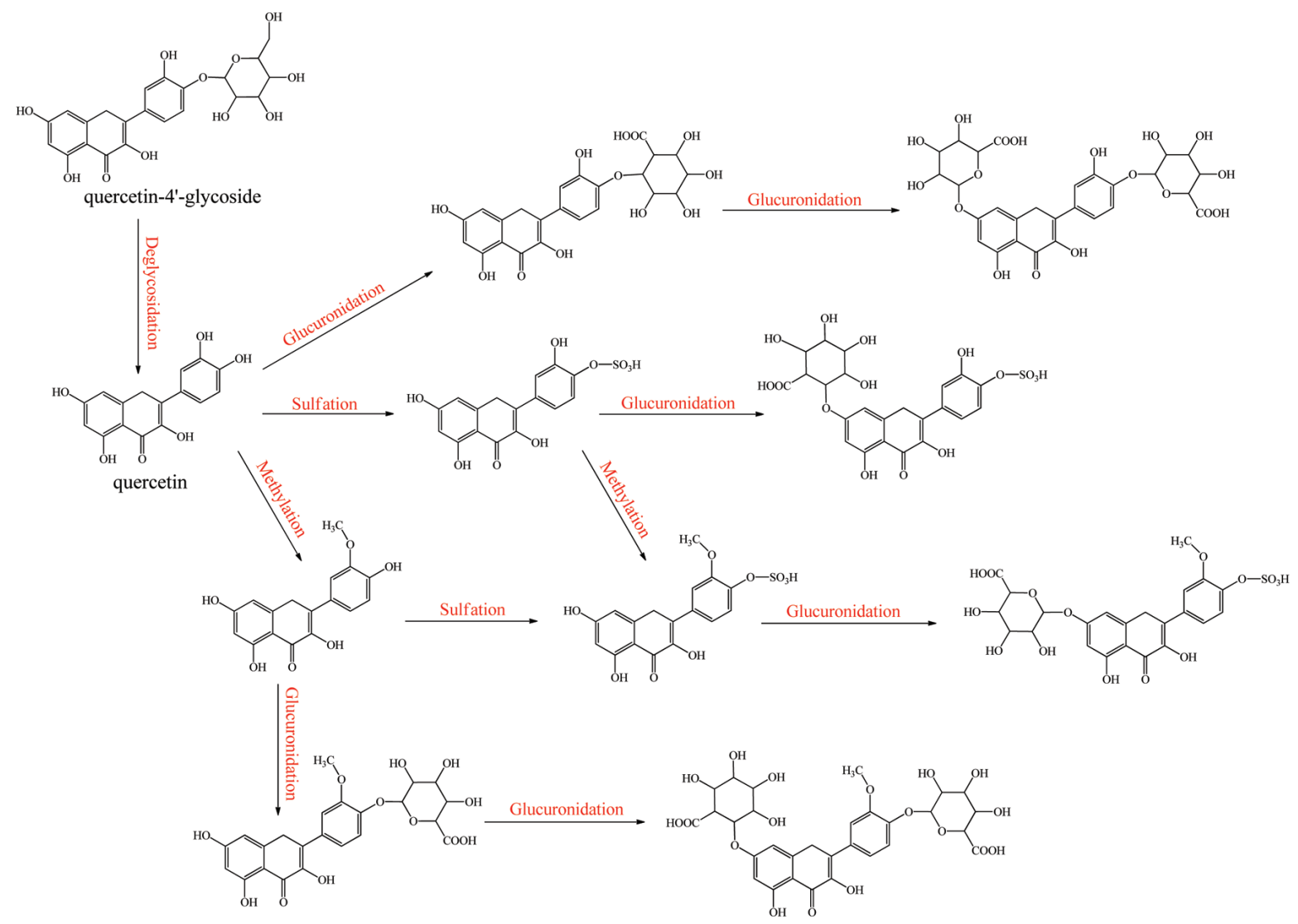

Figure 3. Metabolism of quecetin monoglycoside.

tant tissues for phase I and phase II metabolism of phenolic compounds; other tissues including muscle and brain are also commonly involved and investigated. In the small intestine of humans, the expressed total amount of CYP-450s is less than $1 \%$ of that in hepatic tissues. COMT and UGTs are extensively expressed in various organs mentioned (Galetin et al., 2010; Merello et al., 1994). Similar to the distribution of CYP-450s, the highest expression of COMT is present in liver, followed by gastrointestinal tract, kidneys, and other tissues (O'Leary et al., 2003). In contrast, the expression level of the two main phenol SULTs (SULT 1A1 and SULT 1A3) is the highest in the small intestine, followed by liver, stomach, and colon. For UGTs, UGT 1 and 2 are the two major subgroups in catalyzing phenolic glucuronidation expressed on either liver, gastrointestinal tract, kidney or other organs (Fisher et al., 2001; Rowland et al., 2013). More distribution information has been summarized in Table 10. In normal living cells, the CYP-450s, UGTs, and SULTs are expressed on the cytosolic face of the endoplasmic reticulum (ER), the luminal face of ER, and cytosol, respectively (Galetin et al., 2010; Wu et al., 2011). COMT was reported to be the highest expression in liver and kidney, which could be divided into cytosolic soluble COMT (S-COMT) and rough endoplasmic reticulum membrane-bound COMT (MB-COMT). The former is the predominant form in the cells responsible for xenobiotic methylation (Crespy et al., 1999; Nissinen et al., 1988). Among rat tissues, Donovan et al. (2001) concluded that liver was primarily responsible for sulfation and methylation of flavonoids and small intestine was the organ in which mainly glucuronidation and methylation of flavonoids took place. However, in humans, sulfation should mainly happen in the small intestine rather than the liver due to a higher expression level of phenol SULTs in the intestine. This difference was confirmed in a later study (van der Woude et al., 2004). For phenolic glycination, the synthesis site of hippuric acid/hydroxyhippuric acid almost entirely occurs in the human liver (Toromanović et al., 2008).

\subsection{Phase II metabolism in enterocytes}

After being absorbed phenolics enter into the intestinal and hepatic cells; original phenolic glycosides may be hydrolyzed into aglycones by an intracellular $\beta$-glucosidase, called broadspecificity cytosolic $\beta$-glucosidase which is rich in mammalian liver, kidney and small intestine (Day et al., 1998; Hays et al., 1996). However, the rate and extent of deglycosylation are still variable due to the distinct structure of the phenolic aglycones and the position/nature of the sugar substitutions. Specifically, quercetin-4'-glucoside, naringenin-7-glucoside, apigenin-7-glucoside, genistein-7-glucoside, and daidzein-7-glucoside can be rapidly deglycosylated by both the small intestine and the liver $\beta$-glucosidase extracts. Herein, genistein-7-glucoside has a higher affinity for $\beta$-glucosidase than quercetin- 4 '-glucoside thus the former shows a higher deglycosylation rate, whereas some other glycosides such as quercetin-3,4'-diglucoside, quercetin-3-glucoside, kaempferol-3-glucoside, quercetin-3-rhamnoglucoside, and naringenin-7-rhamnoglucoside prefer to remain unchanged (Day et al., 1998). This intracellular reaction is regarded as a vital way to expose hydroxyl group of xenobiotics for further conjugation 


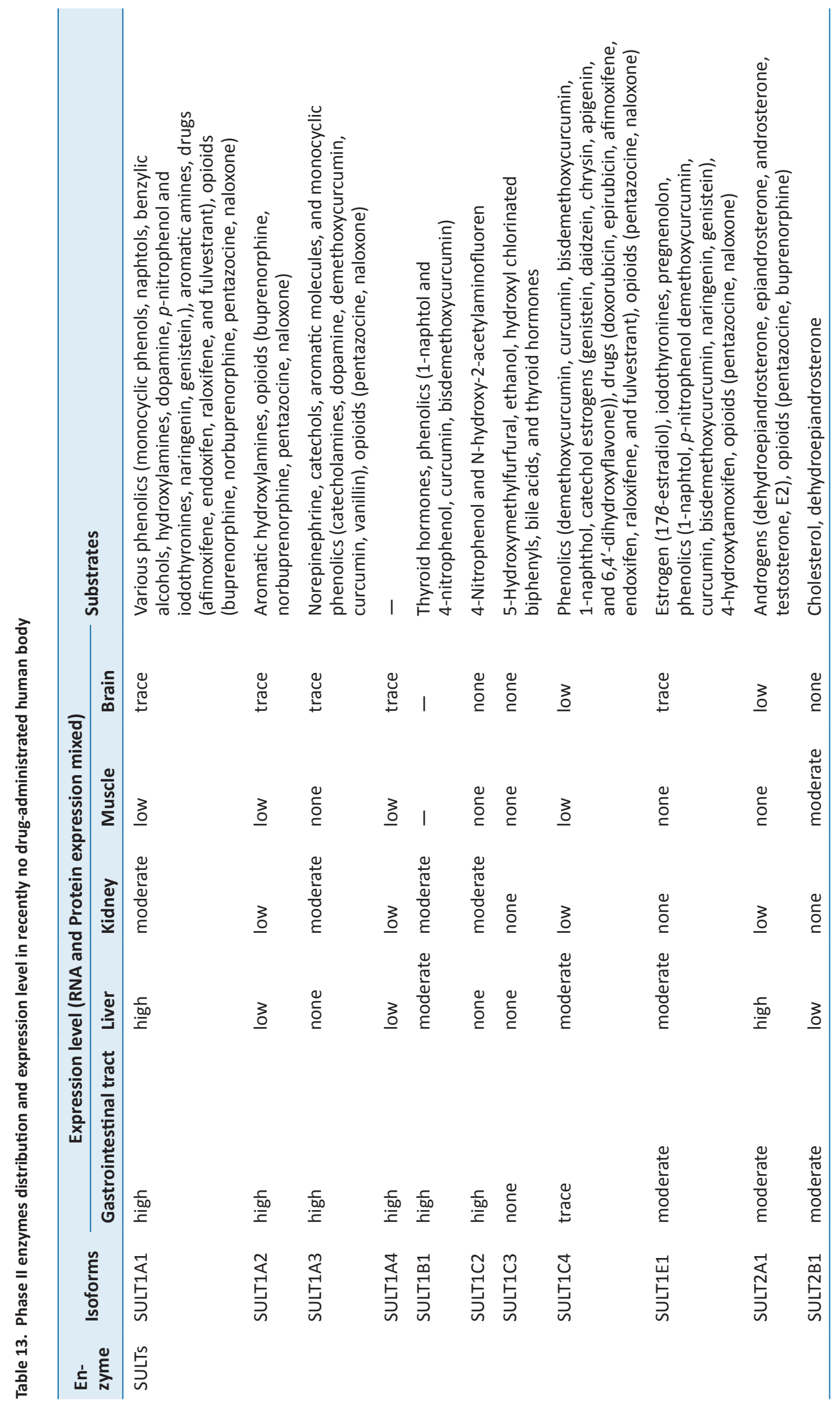




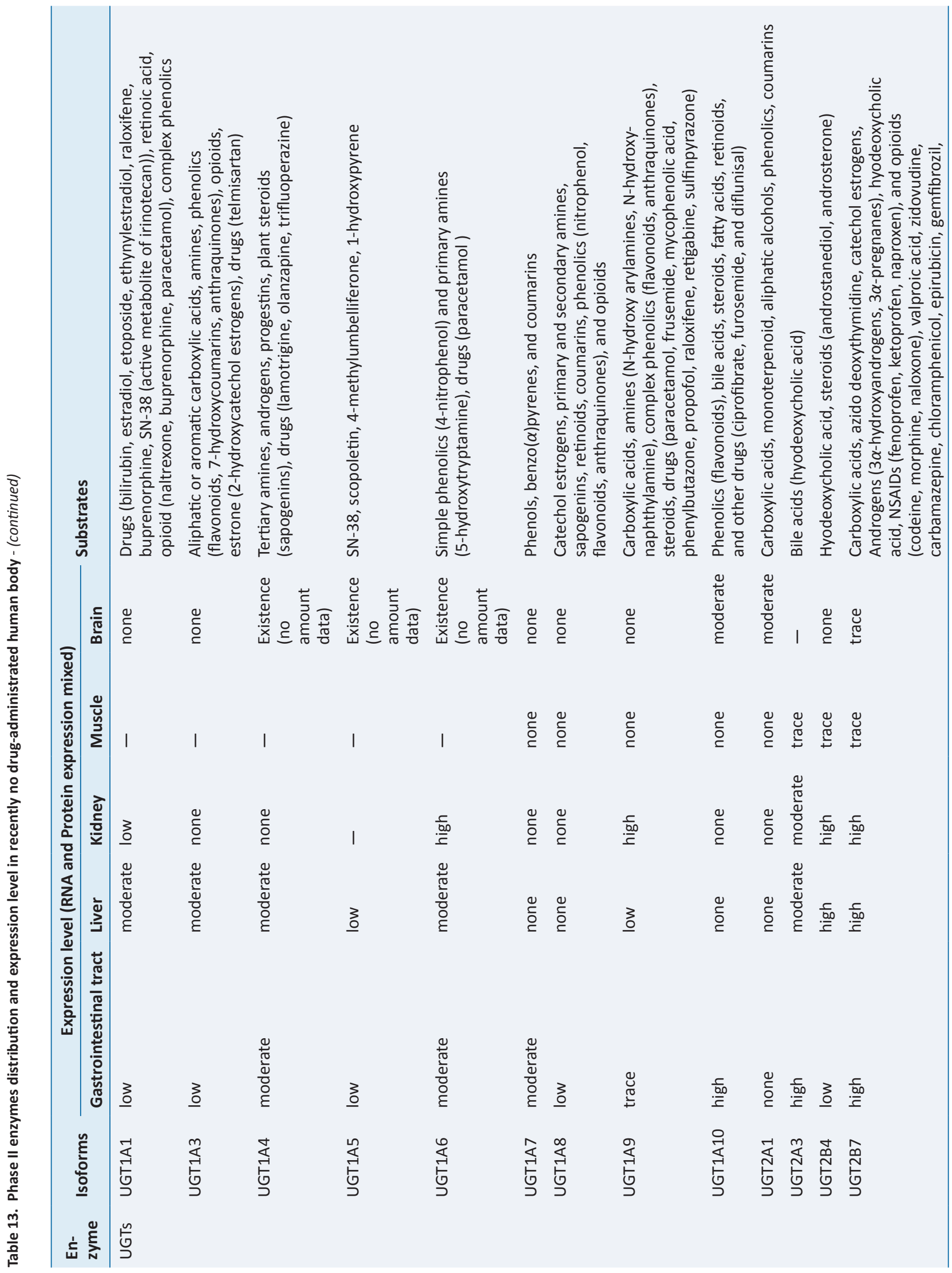




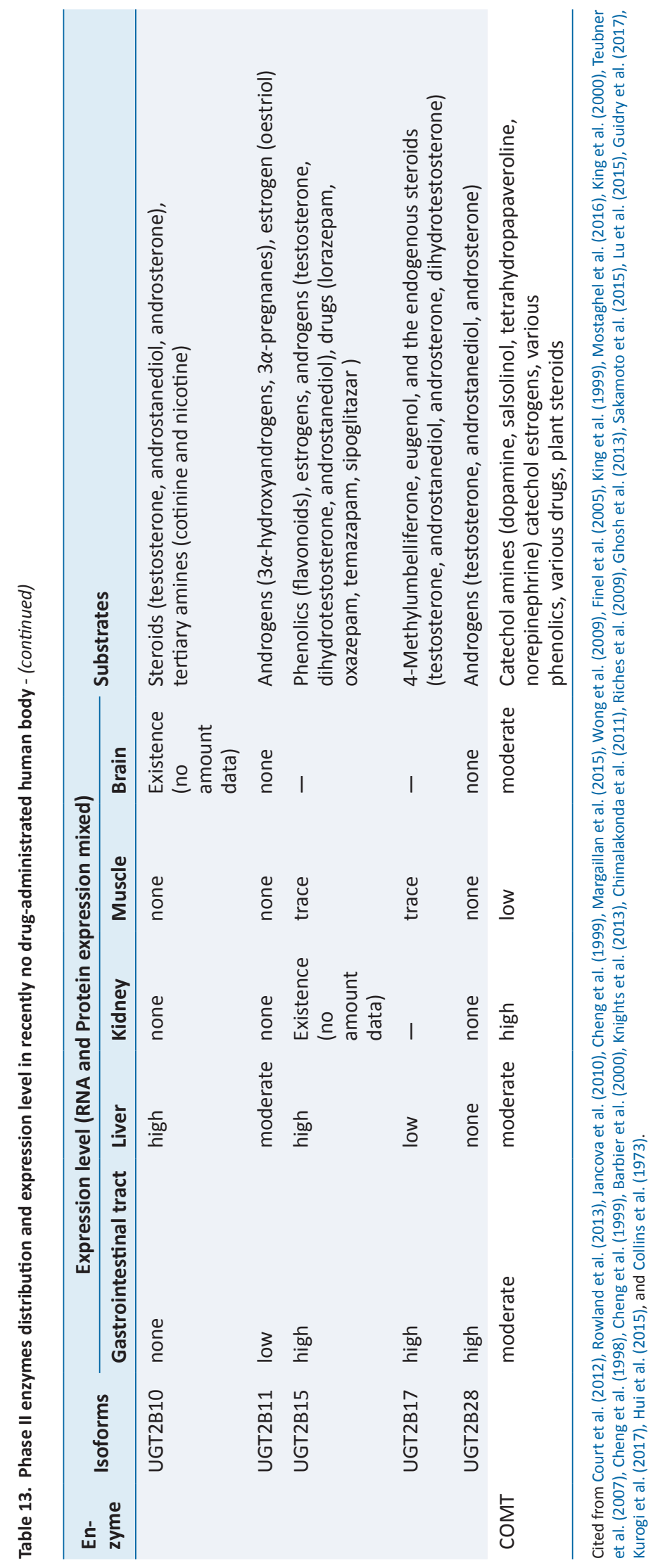




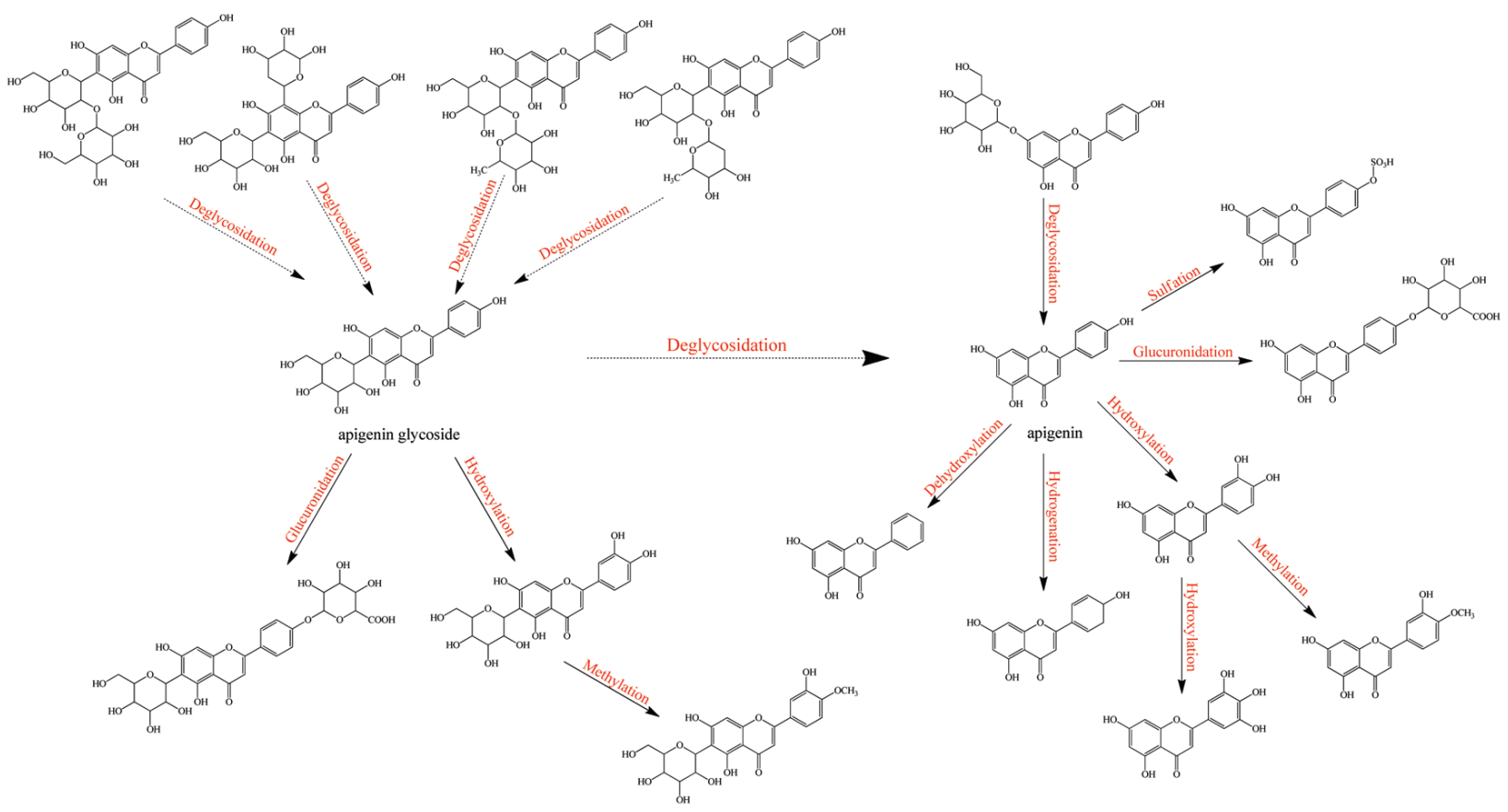

Figure 4. Metabolism of apigenin-C/O-glycosides.

in phase II metabolism.

After deglycosylation, together with original phenolic glycosides and aglycones, a part of aglycones hydrolyzed from glycosides is transported into the blood or/and lymph, whilst others are oxidized or/and conjugated in the gastrointestinal cells, thus easy to undergo ionization. A considerable portion of these phenolic ions would further be conjugated and excluded from enterocytes back to intestinal lumen and undergo enteroenteric recirculation

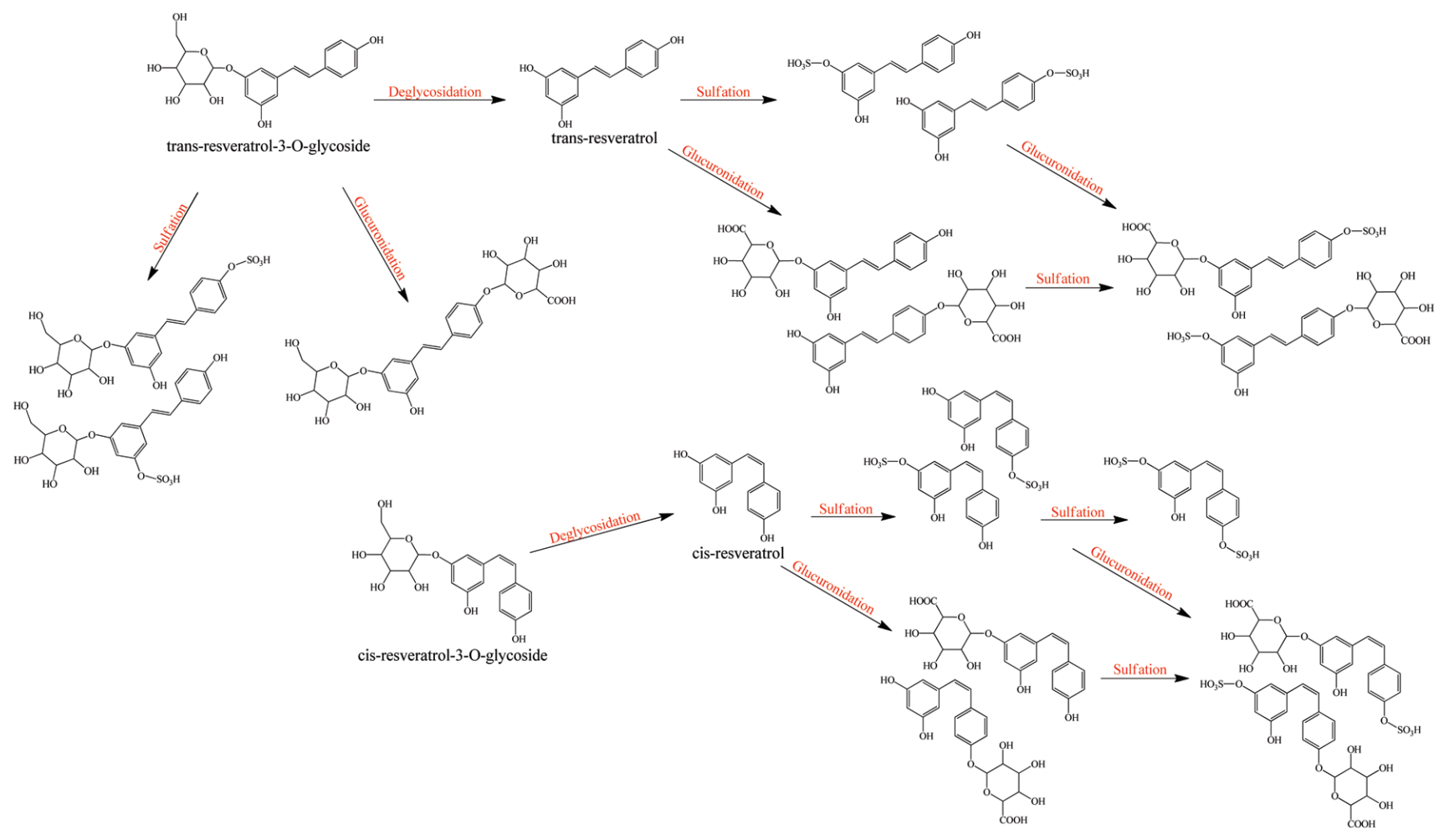

Figure 5. Metabolism of resveratrol and resveratrol glycoside. 


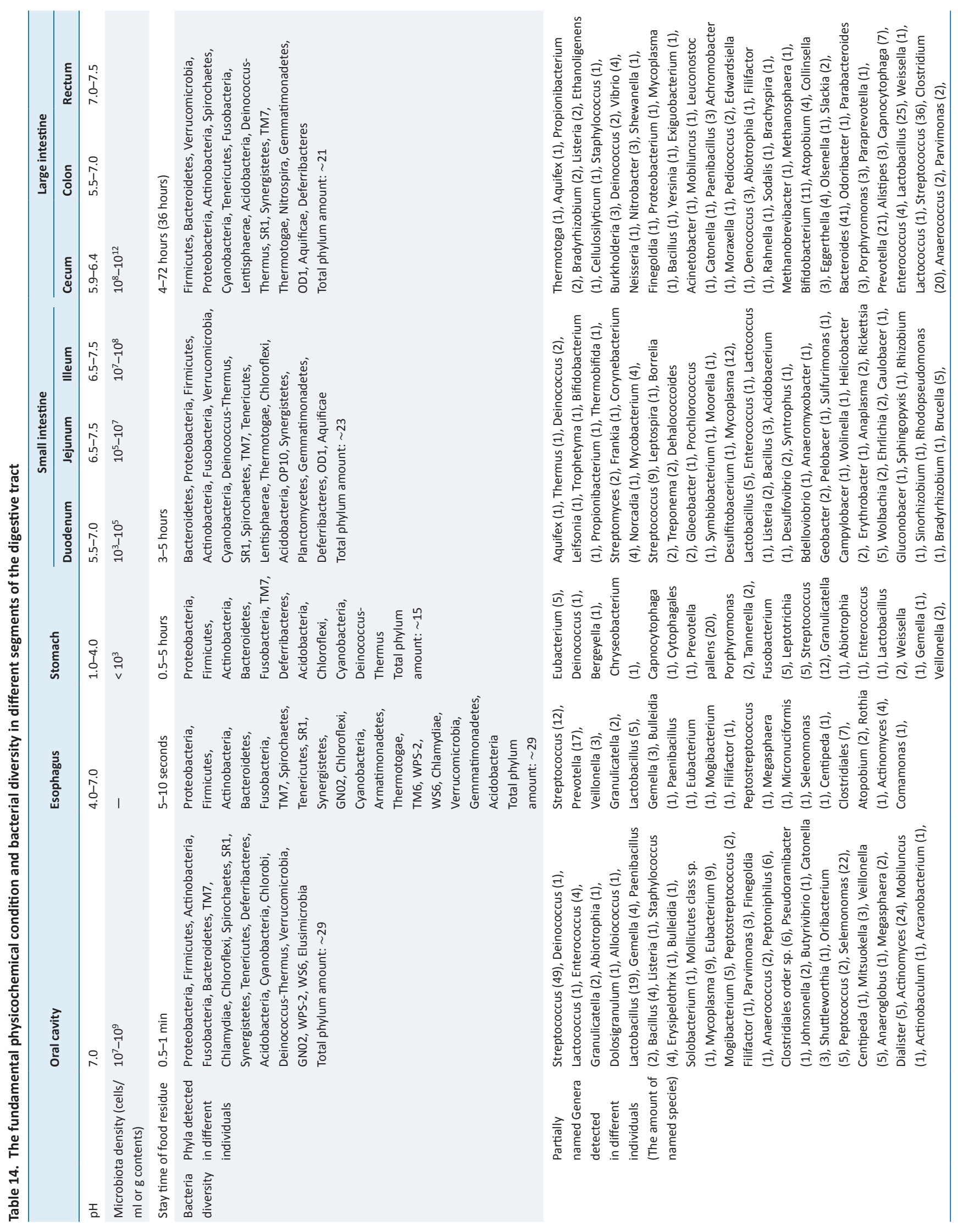




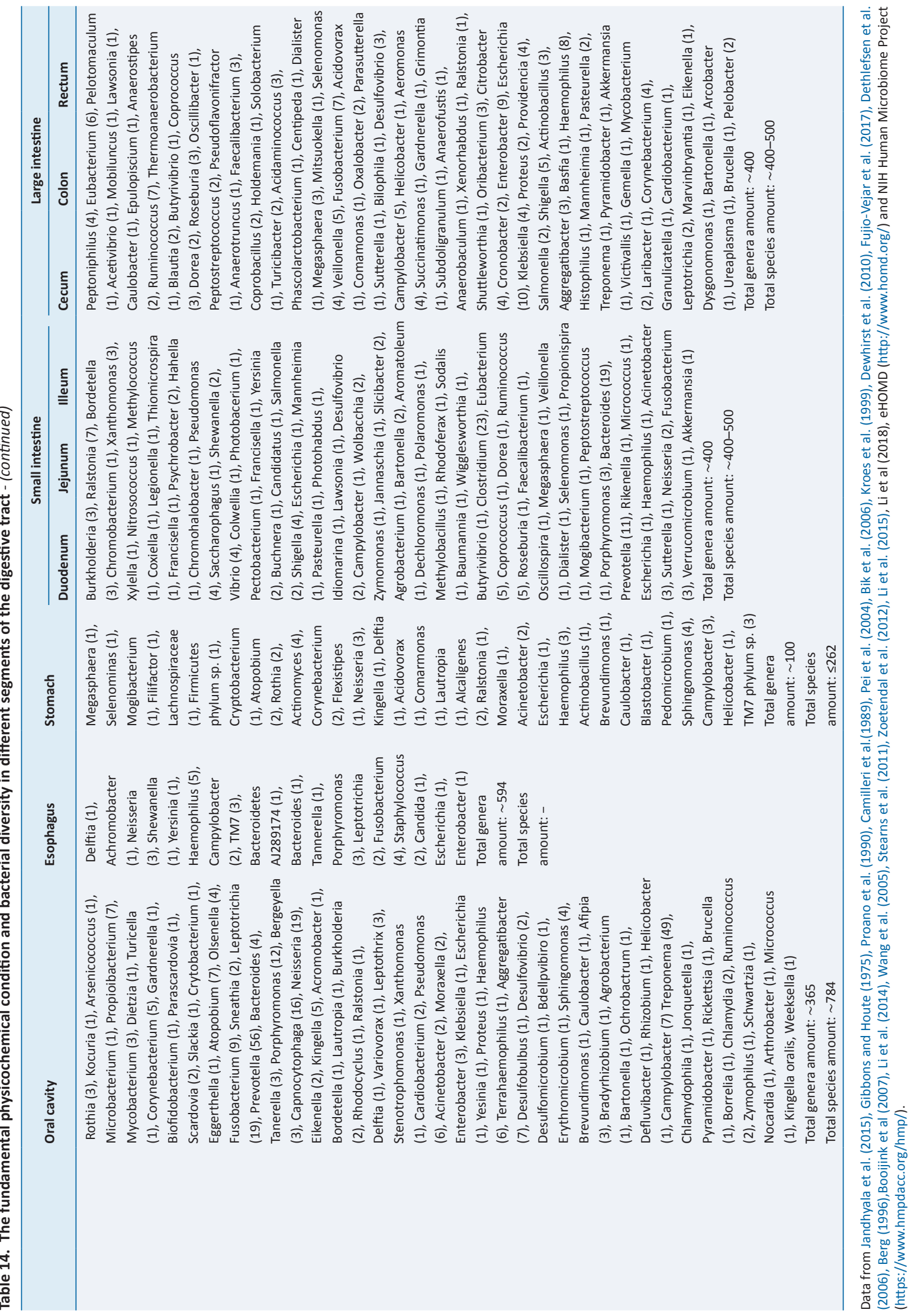




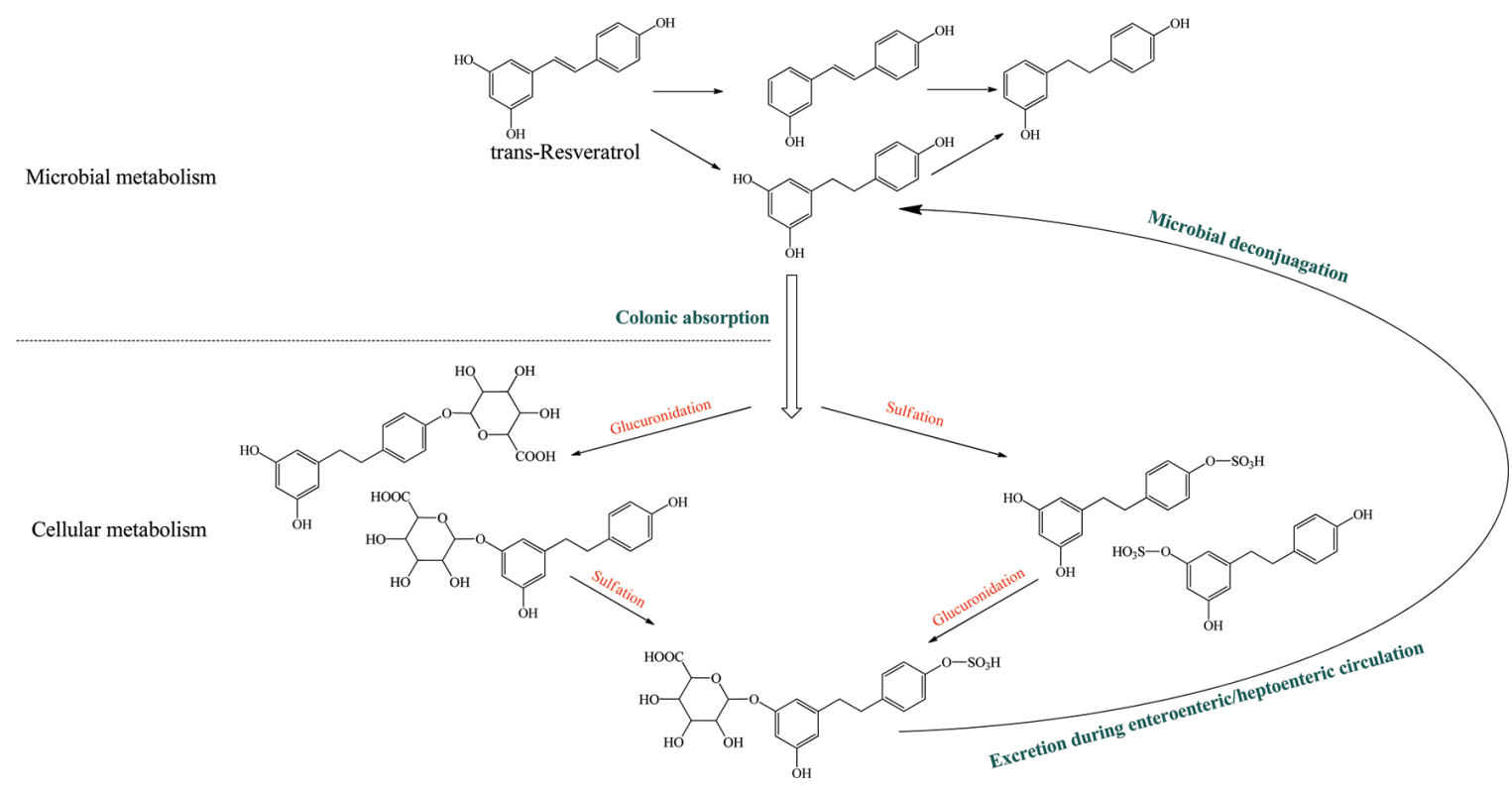

Figure 6. Microbial metablism of resveratrol and corresponding enterohepatic/enteroenteric circulation.

(e.g., about 90 and $52 \%$ of the total absorbed chrysin and quercetin, respectively, were transported back to intestinal lumen as mono-/multiple-conjugates and/or non-conjugates). The form of effluent phenolics varies significantly according to the specific phenolic structures, such as the discrepancy between quercetin (effluent fraction contains 33\% quercetin, 43\% quercetin glucuronidates, and methoxylated quercetin glucuronidates, and $24 \%$ quercetin sulfates) and catechin (effluent fraction is all the original catechin) (Donovan et al., 2001). Then the rest of metabolites (about $14.3 \%$ for quercetin) and original phenolics (aglycones or glycosides) arrive at the liver through portal vein before the next stags that deliver them in systemic circulation or directly enter into systemic circulation with lymph (Crespy et al., 1999; Galijatovic et al., 1999; Walle et al., 1999).

\subsection{Phase II metabolism in hepatocytes}

In the hepatocytes, the original phenolic and enterocytes metabolites are further metabolized via deglucuronidation as well as glucuronidation, sulfation, methylation, glycination, and phase I metabolism. According to Day et al. (2000a) and O'Leary et al. (2003), the dominant quercetin metabolites from enterocytes, quercetin 7-O-glucuronides, and 3-O-glucuronides, enter into the liver where these glucuronides would simultaneously be methylated by COMT and deglucuronidated by $\beta$-deglucuronidase. The original aglycones and aglycone hydrolyzed from quercetin glucuronides may be glucuronidated or re-glucuronidated to $3^{\prime} / 4^{\prime} / 5 / 3 / 7-\mathrm{O}$-glucuronides (the affinity of UDP-glucuronosyltransferase of quercetin hydroxyl groups followed the order $4{ }^{\prime}->3^{\prime}->7->3$-, although the maximum rate of formation was for the 7-position, followed by the 3 '-position). Presently, there is no study to verify whether the cycle of glucuronidation and deglucuronidation is involved.

The methylation and sulfation happen at $3^{\prime} / 4^{\prime}$ (mainly $3^{\prime}-$ )-position and $3^{\prime} / 4^{\prime} / 7$-position (mainly), respectively (O'Leary et al., 2003). A similar conjugating situation was found in catechin metabolism (Donovan et al., 2001). Finally, a large percentage of these phenolic metabolites produced or transited by the liver is secreted through the bile duct into the upper part of the duodenum and proceed to enterohepatic recirculation. For example, about $40 \%$ of the absorbed apigenin was excreted in bile fluid as conjugates (mainly 3'-O-methylated glucuronides) (Cai et al., 2007). The left-over in the liver will flow into the rest of the body with blood; for quercetin, the major forms in systematic circulation are 3'-O-methyl glucuronyl sulfate $(91.5 \%)$, followed by glucuronides and methoxylated glucuronides of quercetin (8.5\%) (Morand et al., 1998). As for quercetin-4'-glucoside, methylquercetin glucuronyl sulfates are major metabolites in the blood and quercetin diglucuronides are the major metabolites in the gut, liver, and kidney (Graf et al., 2005). For catechin, the main plasma metabolites are glucuronide, methyl, and 3'-O-methyl glucuronides, followed by sulfates (Donovan et al., 2001). No matter in enterocytes or hepatocytes, sulfation, methylation, and glucuronidation proceed fully and it could theoretically produce 7 categories of phenolic metabolites including methylquercetin, quercetin sulfate, methylquercetin sulfate, quercetin glucuronidate, methylquercetin glucuronides, quercetin glucuronyl sulfates, and methylquercetin glucuronyl sulfates.

\subsection{Phase II enzymatic kinetics}

Cai et al. (2007) found that the formation rate of apigenin glucuronidation was much faster in both human and mouse liver microsomes than that of its sulfation. However, the sulfate products were the main metabolites recovered in normal mammalian metabolism (Jakoby, 2012). As an example, sulfate conjugation of low-dose chrysin occurred at a rate twice that of glucuronic acid conjugation in both Caco-2 and HepG2 cell lines (Galijatovic et al., 1999). The explanation is that the expression of SULTs was much lower than UGTs, at low doses of xenobiotic; sulfate conjugates are predominant products. However, when phenolics were ingested in high quantity, the relatively lower amount of SULTs and high substrate dose resulted in a rapid saturation of SULTs. Thus phenolics have to be catalyzed by UGTs, which actually leads to greater production of glucuronidates (Gradolatto et al., 2004). Similarly, even if COMT shows a similar catalytic rate compared to that of SULTs 


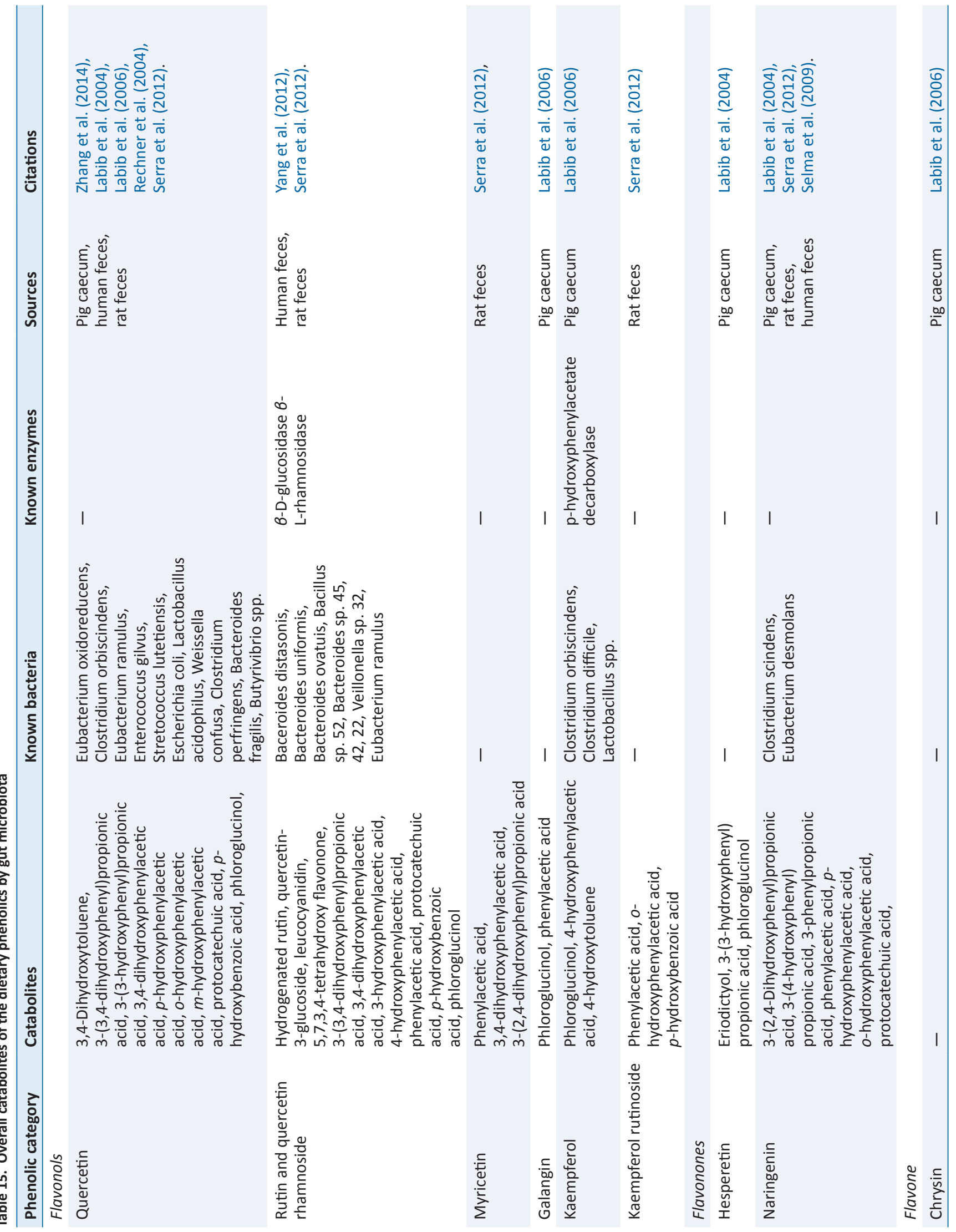




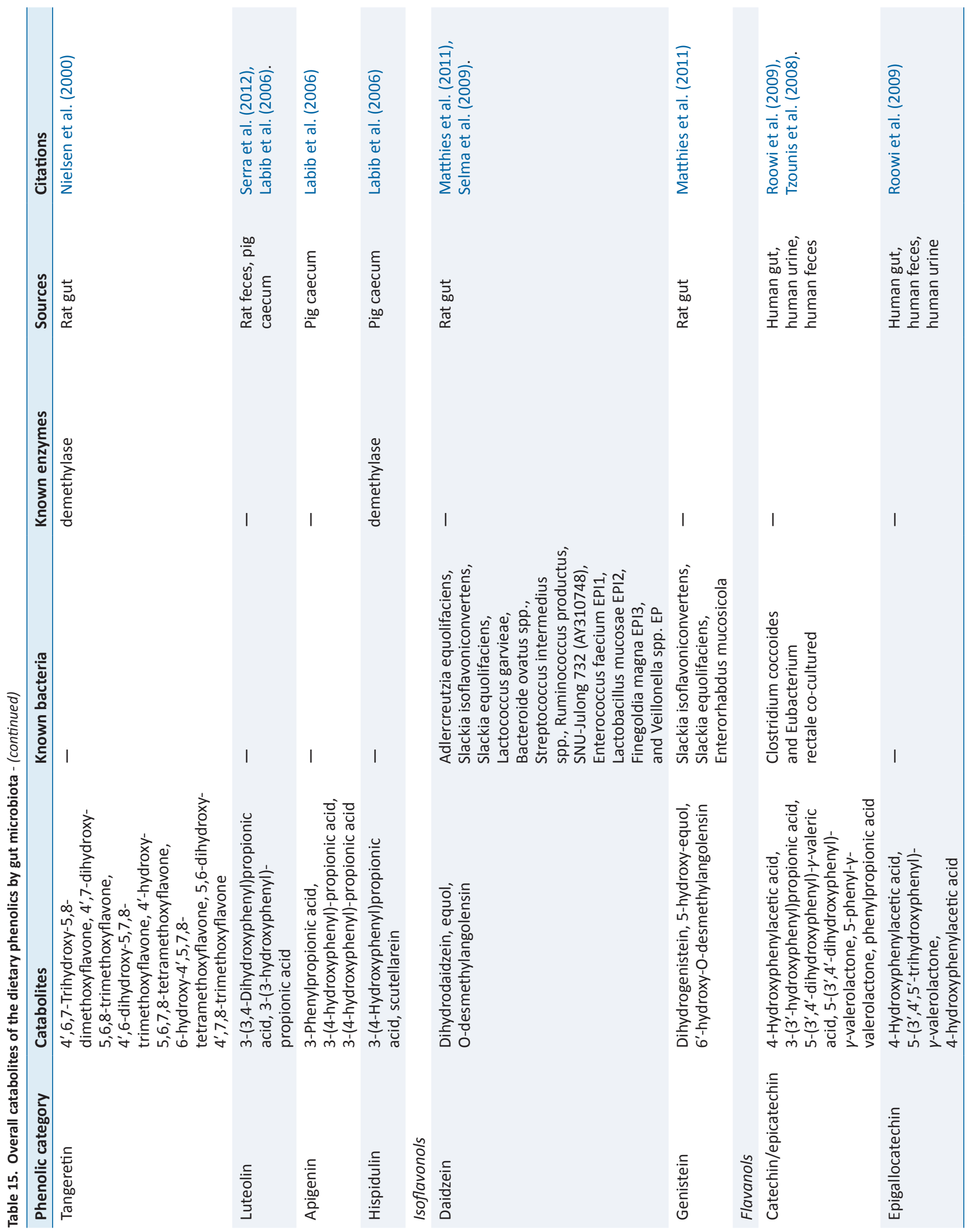




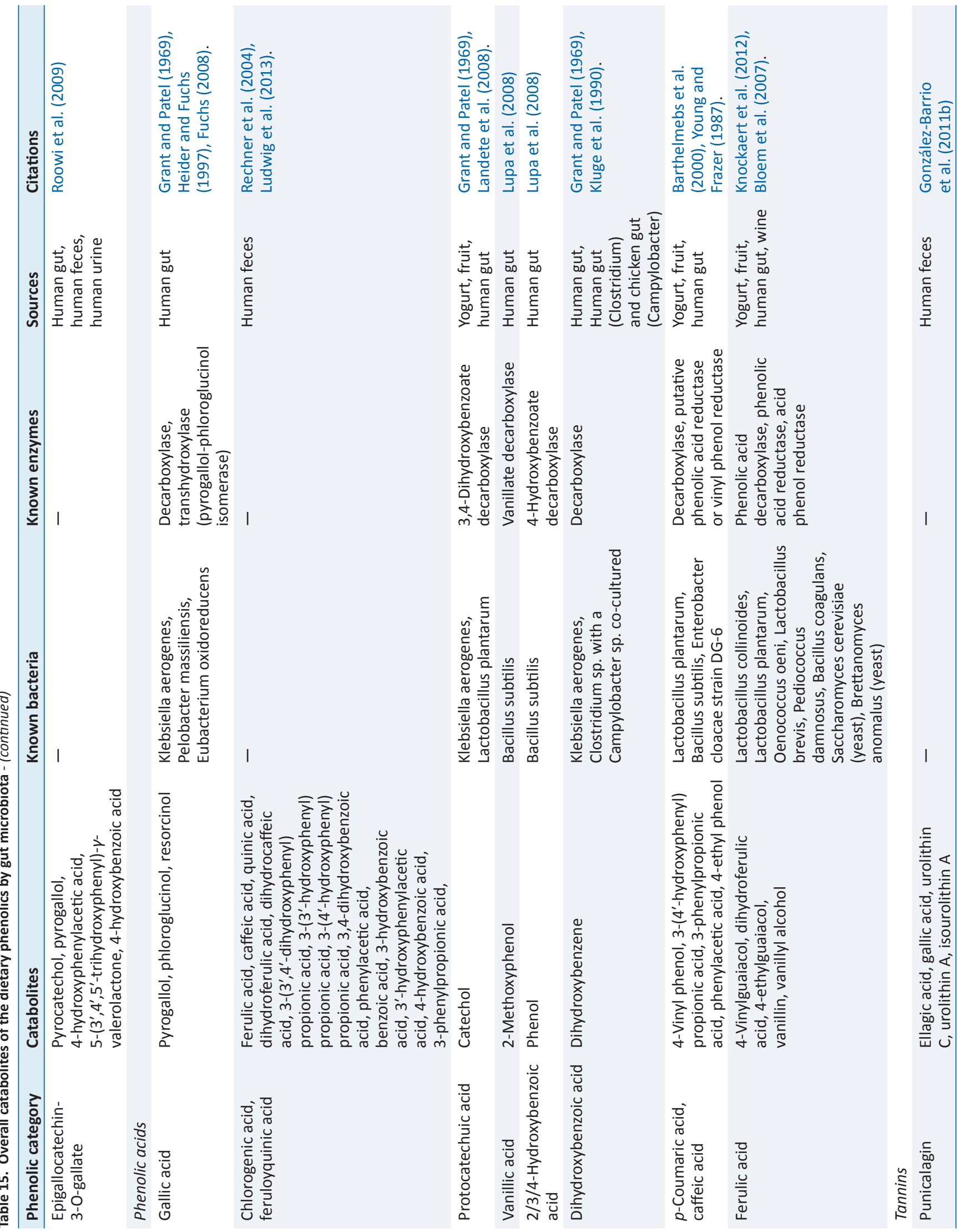




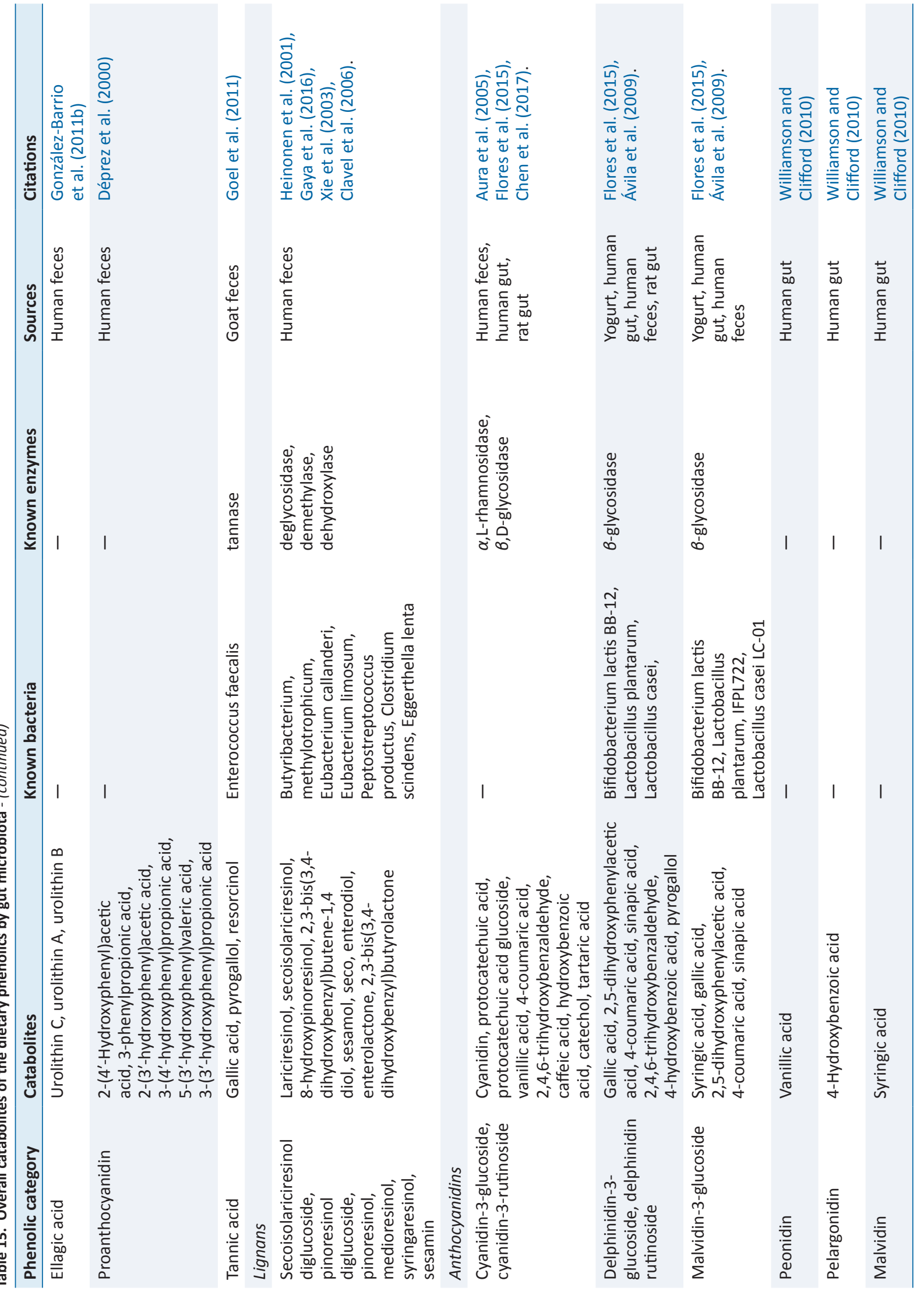




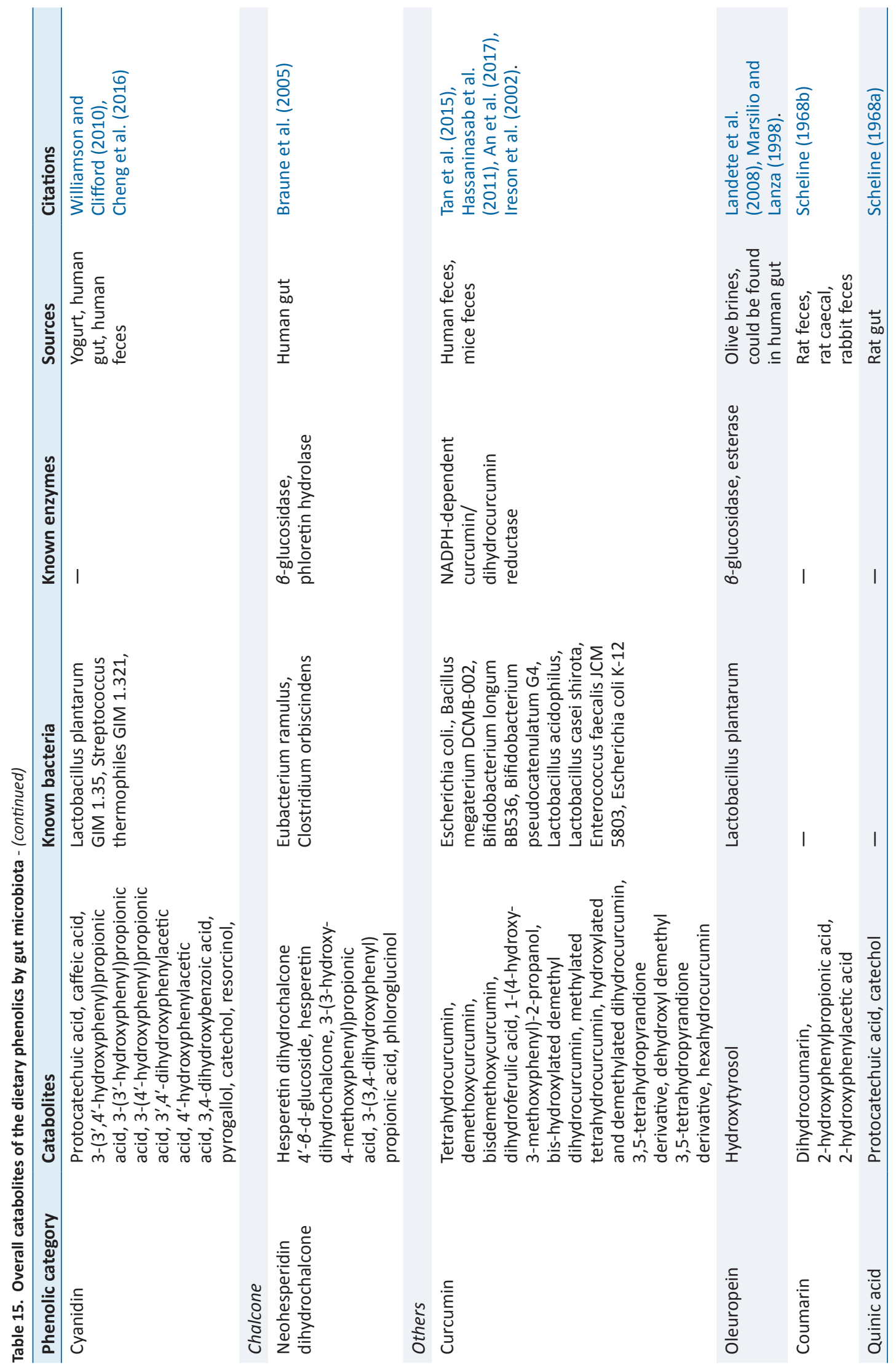


overall (Tables 11, 12), the lower expression of COMT might limit the yield of methyl conjugates. Furthermore, the substitution sites of sulfation $\left(3^{\prime} / 4^{\prime} / 7\right)$ and methylation $\left(3^{\prime} / 4^{\prime}\right)$ are more similar than those of glucuronidation $\left(7 / 5 / 3 / 3^{\prime} / 4^{\prime}\right)$. Thus all these factors might demonstrate the phenomenon that the percentage of phenolic glucuronidates is always the highest among phase II metabolites when high-dose phenolics is administrated. In in vivo metabolism, dozens of these known enzymes may catalyze phenolics in a competitive and random way rather than in the fixed steps even if they have discrepancies in both total amount and reaction rate. This results in metabolites produced in diversified conjugated position and to different extent, and thus much more metabolites are generated than proposed above (Fig. 3) (Crespy et al., 1999; Graf et al., 2005; Morand et al., 1998). The specific species of metabolites and the extent of metabolism are distinct, depending on enzymatic distribution and expression level in different tissues, along with respective phenolic-enzyme affinity (Table 13, Fig. 3-5). More selectivity and reaction orders of different flavonoids in glucuronidation have appeared elsewhere (Wong et al., 2009).

When phenolics are orally administrated as drugs, before arriving into targeted sites, the activity and amount decline caused by metabolic modification and subsequent efflux in the intestine and liver, known as the first-pass metabolism. During enterohepatic and enteroenteric recirculation, some of the reabsorbed phenolics will enter into the whole circular system, but most of them would still be passed to the large intestine with unabsorbed and bound phenolics. Consequently, the poor digestibility, absorbability, efficient biotransformation, and efflux mechanism yield a considerable amount of phenolics to be fermented by the colon microorganism.

\section{Colonic fermentation}

After digestion and absorption in the small intestine, the rest of the chyme consists of undecomposable residues, unabsorbed soluble ingredients, and enterohepatic/enteroenteric circulation fractions that are passed to the lower intestinal tract and finally proceed to colonic anaerobic fermentation. Compared with the proximal digestive tracts, the colon possesses the highest microorganisms population at a density of $10^{12}$ organisms per gram of luminal content, which is involved in maintaining of host's intestinal homeostasis and a relatively longer stay duration (50-90\% of the total digestion duration), thus acting as the major site for food residue fermentation by gut microorganism (Table 14). These colonic microorganisms are mainly the anaerobic bacteria which consist of more than 500 species and about 100 phyla including Firmicutes (65.7\% of 19,548 classified sequences), Bacteroidetes (16.3\%), Proteobacteria (8.8\%), Actinobacteria (4.7\%) and Verrucomicrobia (2.2\%) (Garrett et al., 2010; Hidalgo et al., 2012; Ley et al., 2008). However, when using all the specific body of data for one group or one species it at best is providing an excellent generalization rather than a strict answer. No matter in any segments of the digestive tract, inter-individual and intra-individual variations in microbial quantity and diversity are significant due to different age, race, gender, health condition, medication usage, dietary habit, and environmental microbiome, most of which have to be individually described and still need to be further investigated (Costello et al., 2009; Cotillard et al., 2013; Xu and Knight, 2015). Literally, gut bacteria and their host mutually benefit from one another; bacteria achieve culture medium and produce/liberate the bioactive substances to the host at the same time. These bioactive products may bind to food matrices and are subsequently hy- drolyzed into free micro-molecules (from bound phenolics to free phenolics, from complex phenolics to simple phenolic acids), or originate from fundamental nutrients but further be metabolized and converted into bioactive compounds (from carbohydrates/protein/fatty acids to short-chain fatty acids and vitamins B/K) (Ramakrishna, 2013). Generally, dietary phenolics flowing into colon mainly exist in the bound or conjugated forms. Therefore, the most common step of phenolic degradation process is deconjugation such as demethylation of ferulic acid, deglucuronidation of quercetin 3-O-glucuronic acid and deglycosylation of quercetin 3-O-glucoside. By the way, the oral cavity also contains a trace amount of deglycosylation products of flavonoids due to the action of the oral microbes (Walle, 2004). The disassociation of $\mathrm{C}-\mathrm{C} / \mathrm{C}-\mathrm{O}$ bonds between phenolic oligomers/polysaccharides and phenolic terminal thereof contributes to the exposure of free phenolics and sugars in the colon and provide the carbon source for bacterial consumption (Mosele et al., 2015; Shahidi and Yeo, 2016). Along with deconjugation, the similar conjugation types of phase II metabolism, sulfation and methylation were also proposed to occur in the opposite direction as the first step of phenolic catabolism because the sulfated and methylated catabolites are detected in the in vitro test, even though the earlier study denied the possibility of methylation by gut bacteria (Aura et al., 2002; Kim et al., 1992; Yang et al., 2012). Of course, in the in vivo test instead, these conjugates are most likely re-synthesized through the colonic reabsorption and in vivo metabolism and subsequently secreted into intestinal lumen during enteroenteric and enterohepatic circulation (Fig. 6).

In the same manner as phenolic absorption in the small intestine, the newly released free phenolics and unabsorbed free phenolics of upper tracts could be absorbed by colonic enterocytes and metabolized through the phase I and II routines, as was explained in the previous section on metabolism. In parallel, upon anaerobic fermentation of released monomers such as glucose, amino acids, and fatty acids, phenolic aglycones in the colon may further be bio-converted through multiple-enzyme effects and finally transformed to simpler phenolics. These decomposed products generally present as (but not limited to) hydroxyphenylpropionic acid, hydroxyphenylacetic acid, dihydroxybenzene, benzoic acid, hydroxybenzoic acid, trihydroxybenzaldehyde, catechol and their derivatives (Table 15). The in vivo metabolism and gut catabolism usually process sequentially on the same catabolite, such as hydroxyhippuric acid, 3-(4'-hydroxyphenyl)lactic acid, glucuronyldihydroresveratrol sulfate and 3'-methoxy-4'-hydroxyphenylacetic acid are the products subjected to glycination, hydroxylation, glucuronidation/sulfation and methylation from hydroxybenzoic acid, 3-(4'-hydroxyphenyl)propionic acid, dihydroresveratrol, and 3',4'-dihydroxyphenylacetic acid, respectively (González-Barrio et al., 2011b; Rotches-Ribalta et al., 2012a). Thus, the final absorbed phenolics are not only the orally administrated phenolics and their in vivo metabolites, but also their gut catabolites and the in vivo metabolites of absorbed catabolites. To a great extent, postprandial long-term bioaccessibility of phenolics is affected by these converted metabolites in the colon. The classical pharmacokinetics, therefore, cannot thoroughly quantify the phenolic bioavailability, namely the in vivo bioavailability of phenolics defined by the concentration of native phenolics and their conjugated metabolites without considering absorbed colonic catabolites is less than the actual value. To be more specific, bioaccessibility of resveratrol ranged from 36.3 to $84.9 \%$ within 24 hours, herein, $8.1-62.7 \%$ of absorbed resveratrol were in the form of microbial metabolites including dihydroresveratrol, 3,4'-dihydroxy-trans-stilbene and lunularin (Bode et al., 2013). The postprandial short-term bioaccessibility of ellagitannins is zero until being slightly metabolized into urolithin $\mathrm{C} / \mathrm{D}$ starting by the jejunum bacteria, followed by 


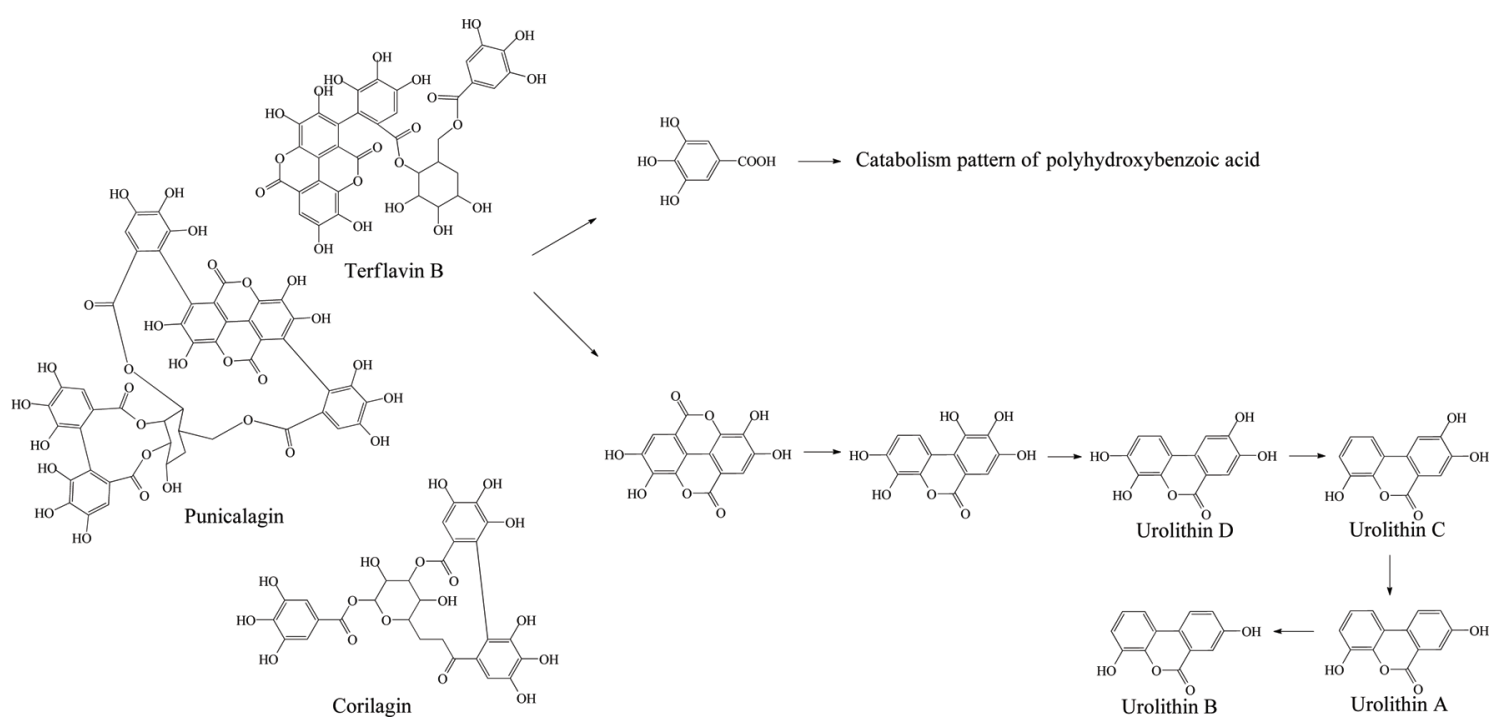

Figure 7. Microbial metabolism of ellagitannin.

massive dehydroxylation into urolithin $\mathrm{A} / \mathrm{B} / \mathrm{C} / \mathrm{D}$ in the colon (Fig. 7) (Gaya et al., 2016).

Meanwhile, the bioactivity of the new metabolites is significantly distinct from their precursors. Gakh et al. (2010) found that dihydroresveratrol, the microbial product of resveratrol, could promote proliferation of MCF-7 cell line at picomolar-range concentration but not by its parental compound. Forester and Waterhouse (2010) demonstrated that gallic acid, 3-O-methylgallic acid, and 2,4,6-trihydroxybenzaldehyde are more effective in reducing the proliferation of Caco-2 than their precursor anthocyanins. Setchel et al. (2002) also reported that equol showed a greater antioxidant activity and higher affinity (10-80 times) to the estrogen receptor $\alpha$ and $\beta$ than the corresponding native isoflavones. As well, Monagas et al. (2009) showed that the most common catabolites from flavonoids, 3,4-dihydroxyphenylpropionic acid, and 3,4-dihydroxyphenylacetic acid, could inhibit $85-98 \%$ production of cytokines (TNF- $\alpha$, IL- $1 \beta$, and IL-6) in lipopolysaccharide (LPS)-stimulated peripheral blood mononuclear cells. On the other hand, the bacterial transformation may result in the loss of native bioactivities, such as desmethylangolensin, another product of daidzein, has no human estrogen-like effect (De Boever et al., 2000). More health effects including inhibition of tumor, mutation, inflammation, oxidative stress, hormone level, blood sugar level and various gut diseases exerted by phenolic gut catabolites in chemical and cellular level has already been reviewed (Aura, 2008; Chiou et al., 2014; Monagas et al., 2010; Tuohy et al., 2012).

In anaerobic degradation of phenolic aglycones, there are two main purposes for gut microbe to modify the side chains and heterocyclic ring of phenolics; the first is to achieve the carbon source and energy to survive, the second is to decrease the original toxicity of phenolics themselves (Mosele et al., 2015). Oleuropein and protocatechuic acid, for instance, could inhibit the growth of Lactobacillus plantarum, but their catabolites including hydroxytyrosol, elenoic acid, and catechol transformed by Lactobacillus plantarum have no inhibitory effect anymore (Landete et al., 2008). On the other hand, two colonic obligate anaerobic bacteria Bacteroides iuniformis and Bacteroides ovatuis can only utilize the sugar moiety of flavonoid glycosides and produce aglycones without any further catabolism (Winter et al., 1989). More interestingly, Butyvriiibrio spp. could catabolize quercetin glycosides into simpler phenolic acids but exerts no catabolic capacity when there is no sugar moiety on aglycone, while Eubacterium oxidoreducens works on both quercetin glycosides and aglycones (Table 16) (Winter et al., 1989). From another point of view, these bacteria with their unique characters could collaboratively decompose dietary phenolics. For example, the Bacteroides spp., one of the most common and large-quantity gut flora, hydrolyze flavonoids glycosides, followed by aglycones' decomposition by Clostridium orbiscindens, Butyrivibrio sp. C3, and Eubacterium oxidoreducens. Other gut bacteria involved in phenolic catabolism are given in Table 15 and also appear in several reviews (Aura, 2008; Braune and Blaut, 2016; Selma et al., 2009). Obviously, distinct principal skeletons of aglycones would be disposed by different enzymes and result in various metabolism pattern. Besides, as already mentioned, the inter-individual variation results in a huge variety in species of individual gut flora and also contributes to a potential difference of the catabolism pattern on the same phenolics. Gross et al. (2010) reported the differences of tea catabolism by the gut flora of 10 feces donors, the catabolites gallic acid and pyrogallol of tea polyphenols ranged from 0.05 to $0.82 \mathrm{mM}$ at 8 th hour and 0 to $0.25 \mathrm{mM}$ at 70 th hour, respectively. After 72 hours in vitro fecal incubation of the red wine/grape juice phenolics, the catabolic intermediates (3-hydroxyphenylacetic acid and phloroglucinol) were absent in several donors' feces sample but were abundantly present within other incubations. In the study of in vitro human fecal incubation of ellagic acid, the catabolites consisting of urolithin $\mathrm{A}$, isourolithin $\mathrm{A}$, and urolithin $\mathrm{C}$ were formed in most of the incubations, exceptionally, one of them could also produce urolithin B (González-Barrio et al., 2011a). Apart from the interindividual variation, intra-individual condition altering including aging, stress, disease and dietary habit also ignites the qualitative and quantitative change of gut flora and corresponding phenolic catabolism. Thus, inter-/intra-individual variation in gut phenolic biotransformation significantly affects phenolic bioavailability and potential bioactivity.

Overall, the principal reactions of phenolic aglycones may include hydrolysis, reduction, and oxidation. In terms of phenolic biotransformation, these reactions could be divided into peripheral and central aromatic metabolism. The peripheral metabolism contains heterocyclic-ring cleavage, de-esterification, de-etherifi- 


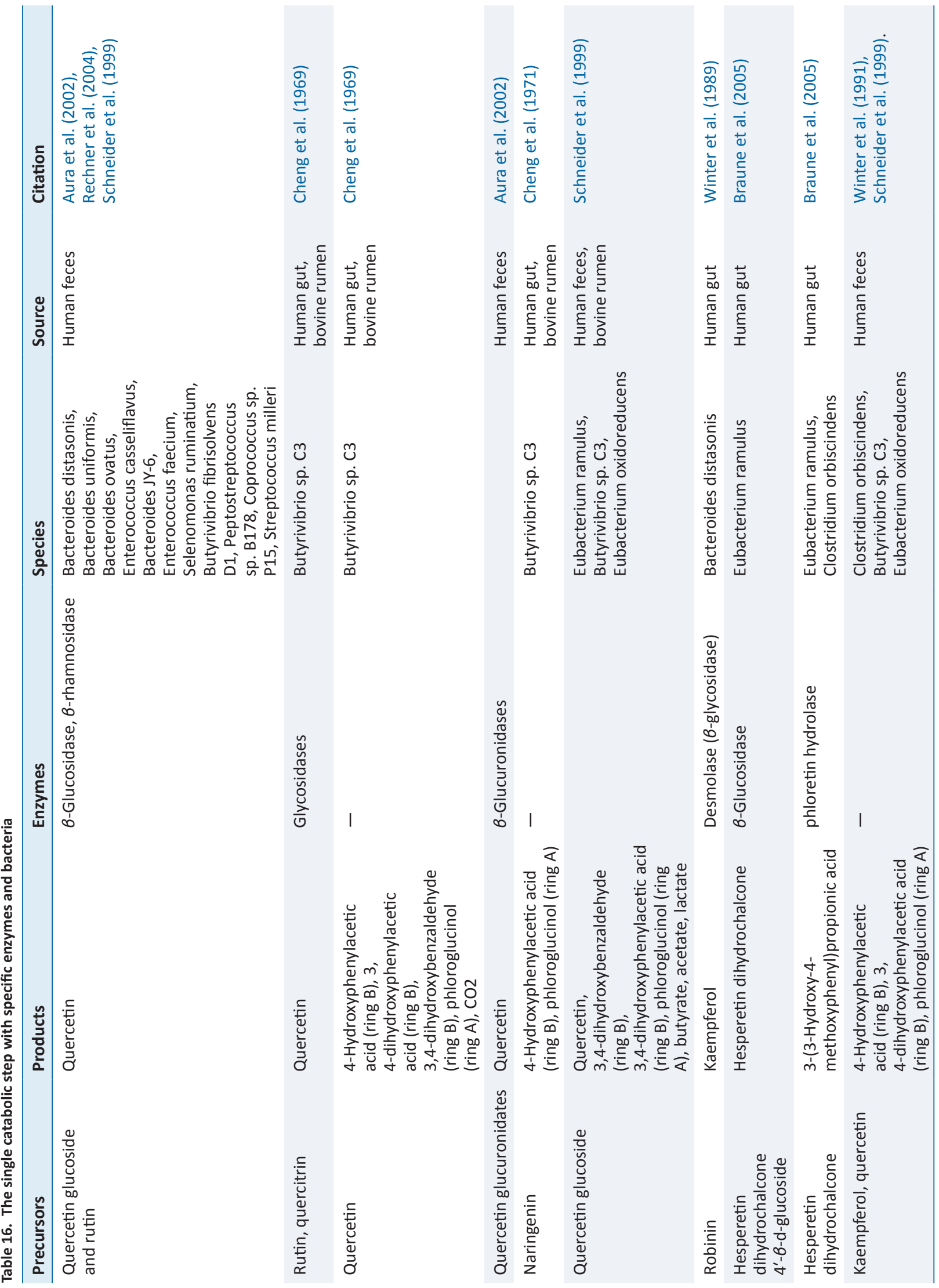




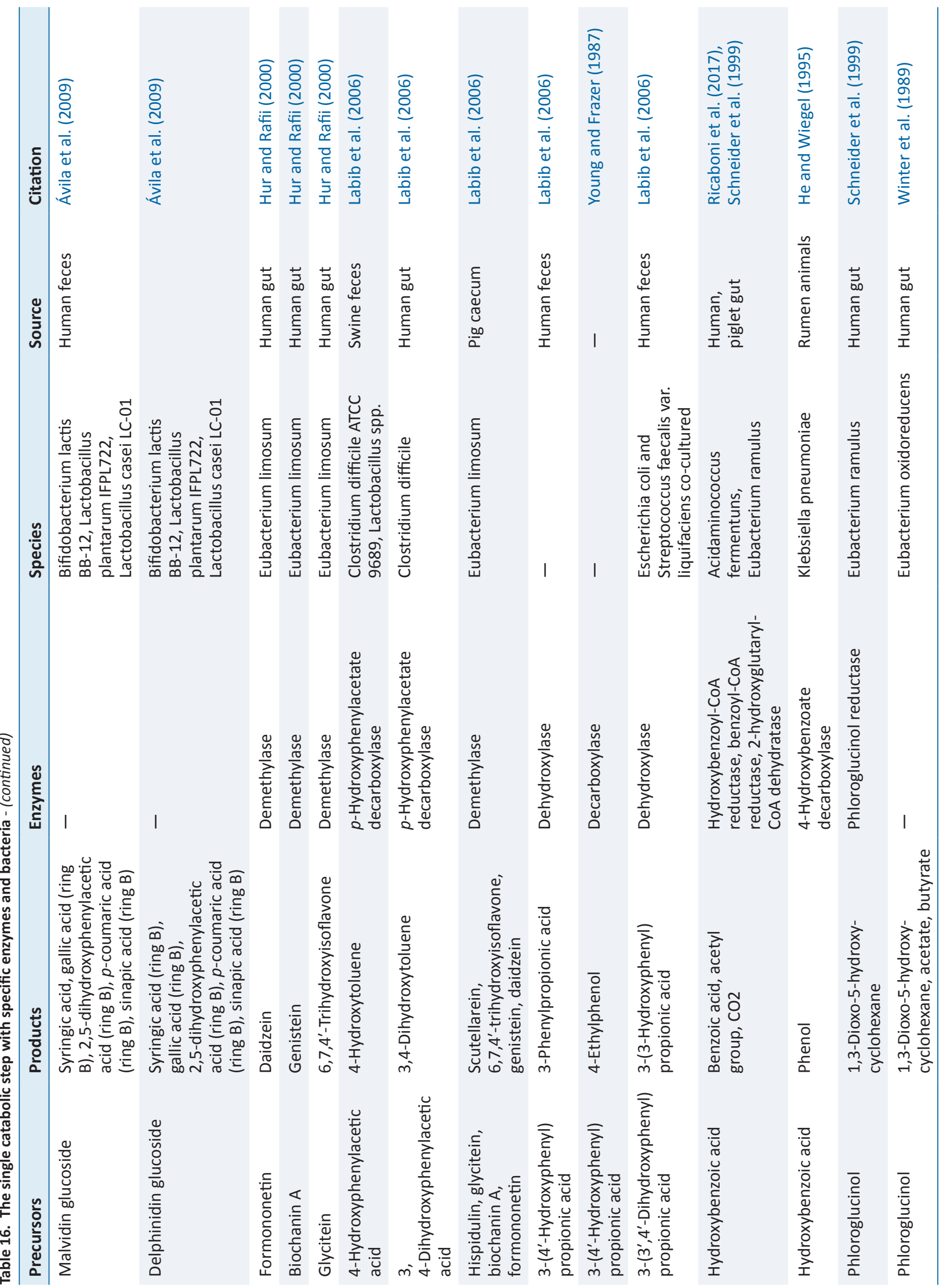




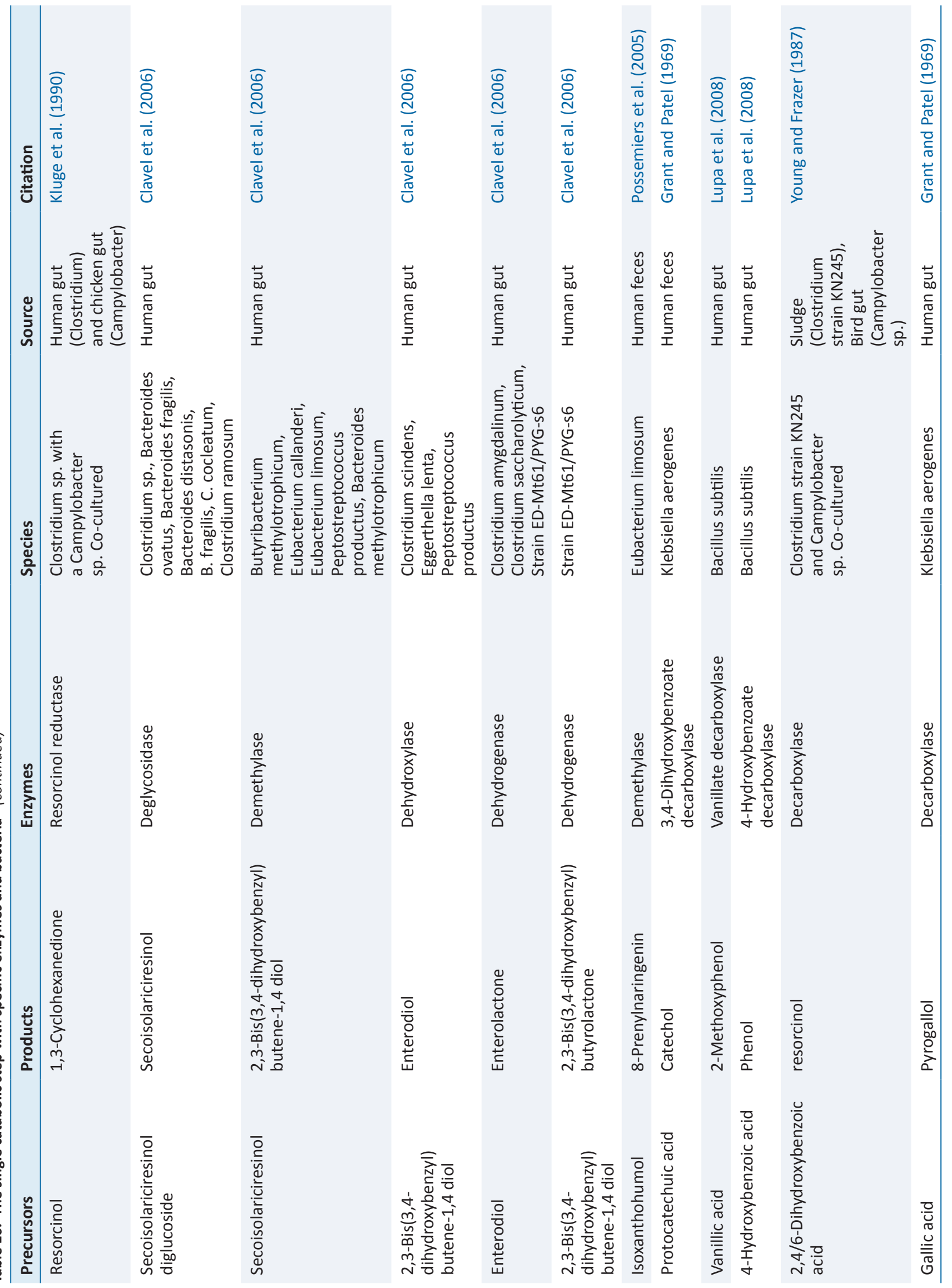




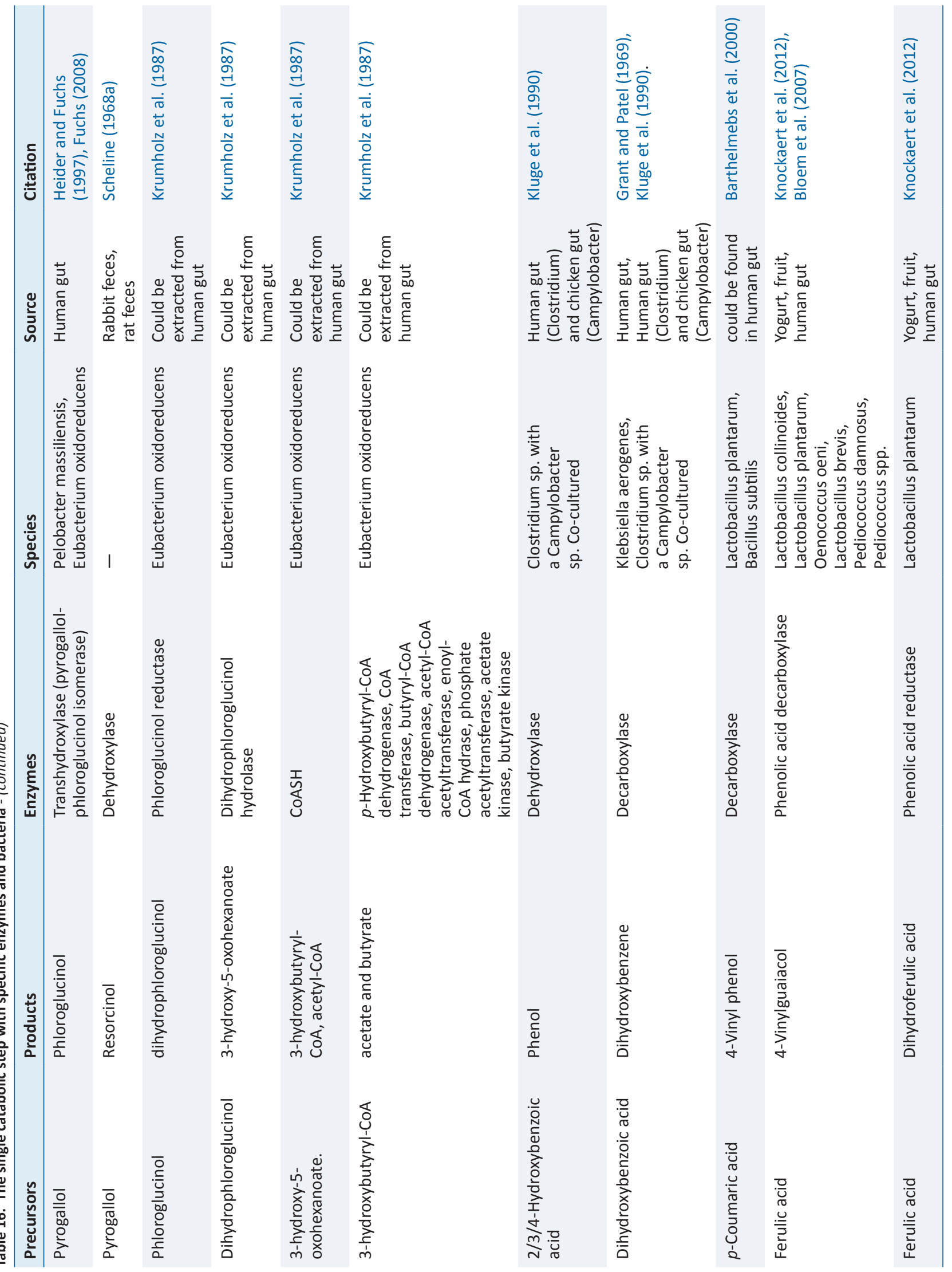




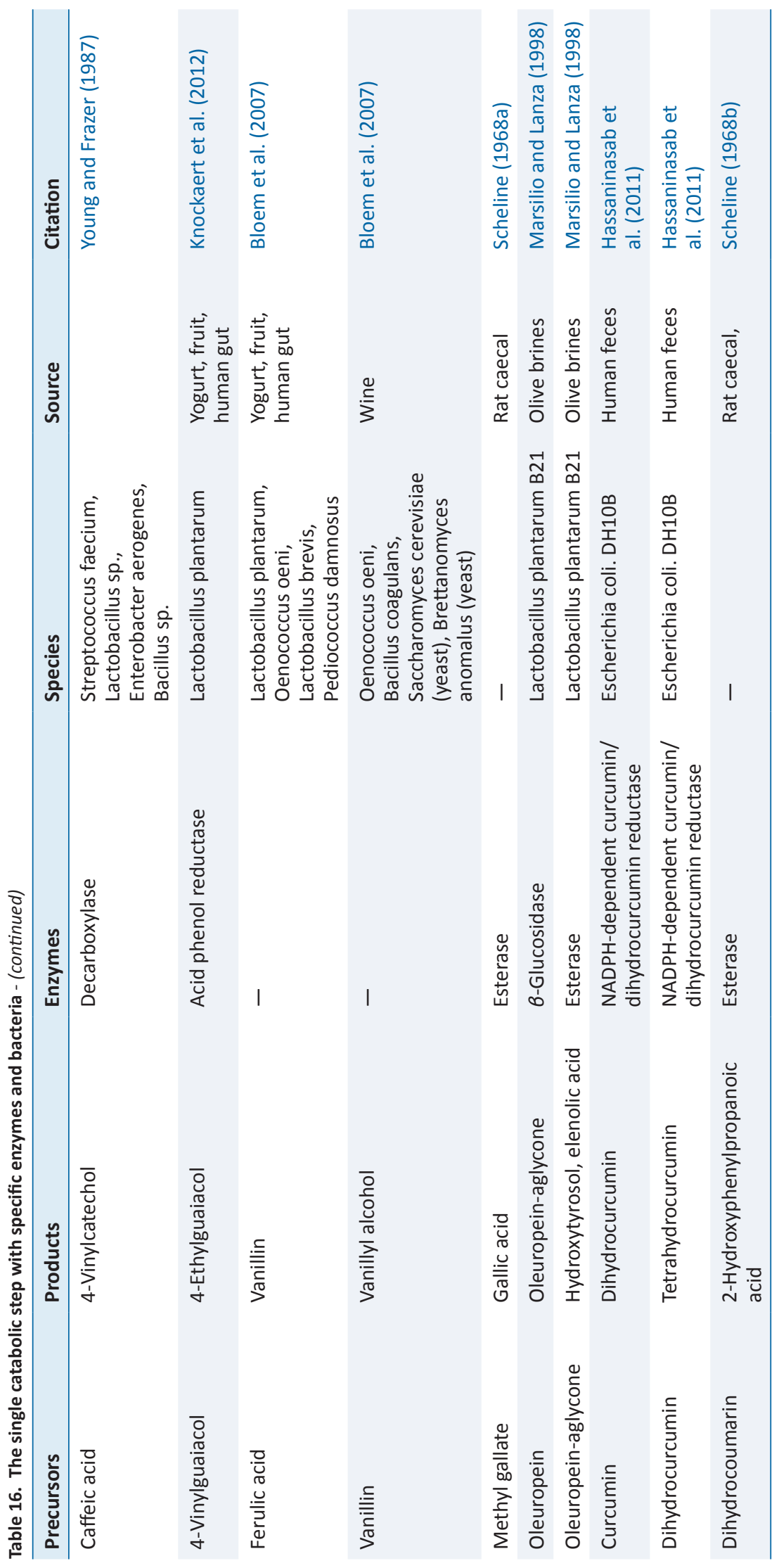




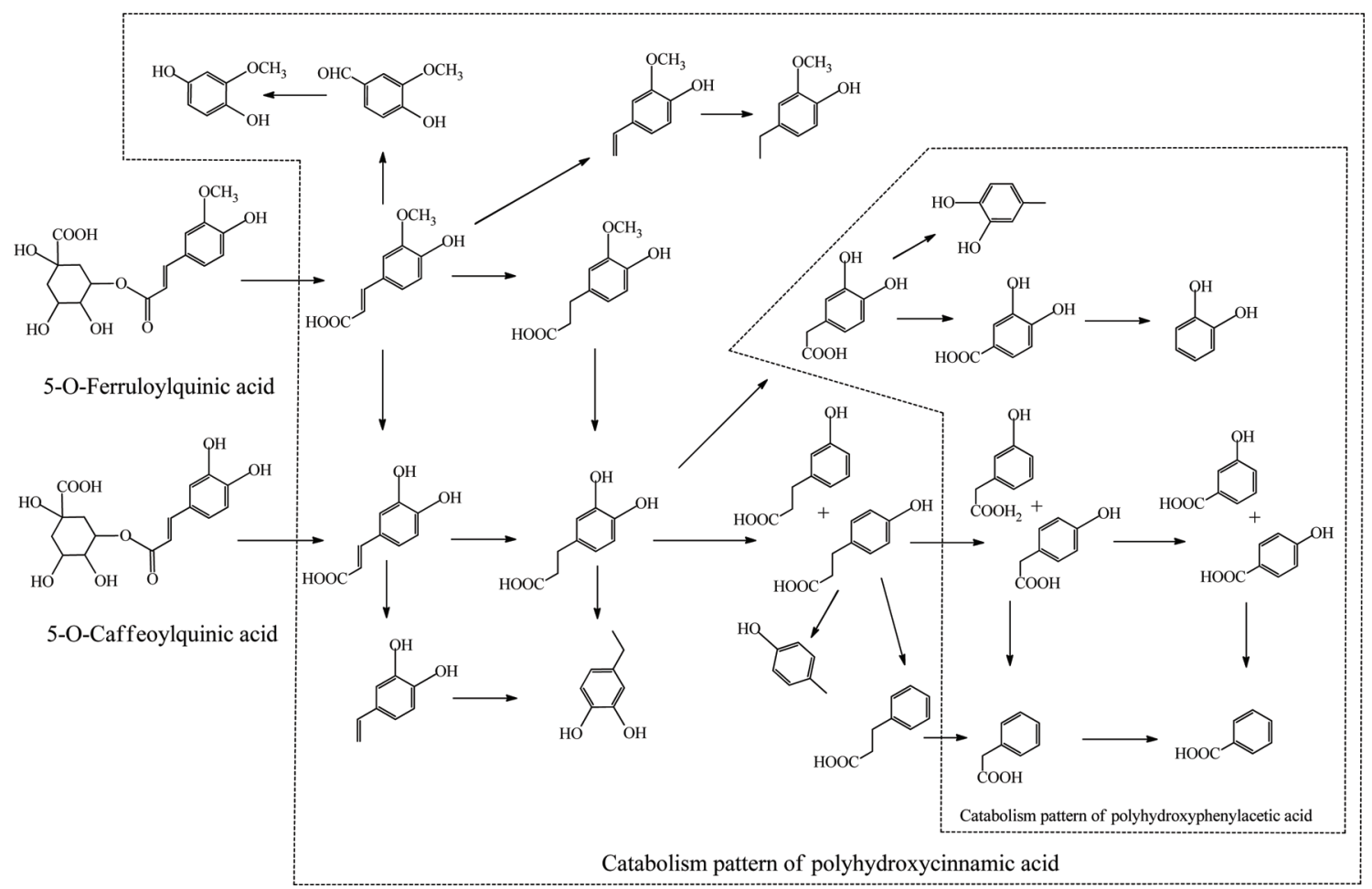

Figure 8. Microbial metablism of cinnamic acid esters.

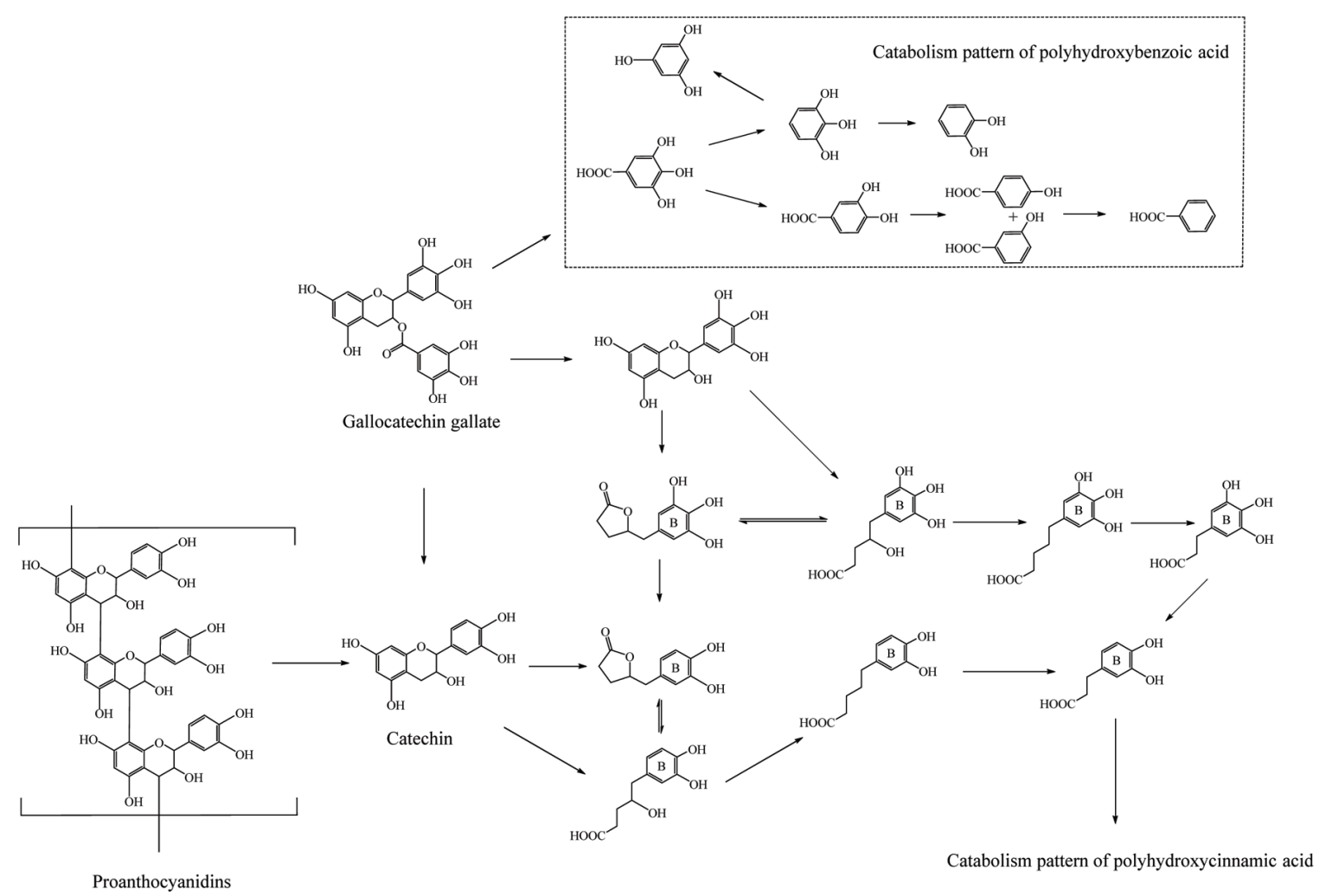

Figure 9. Microbial metablism of proanthocyanin. 


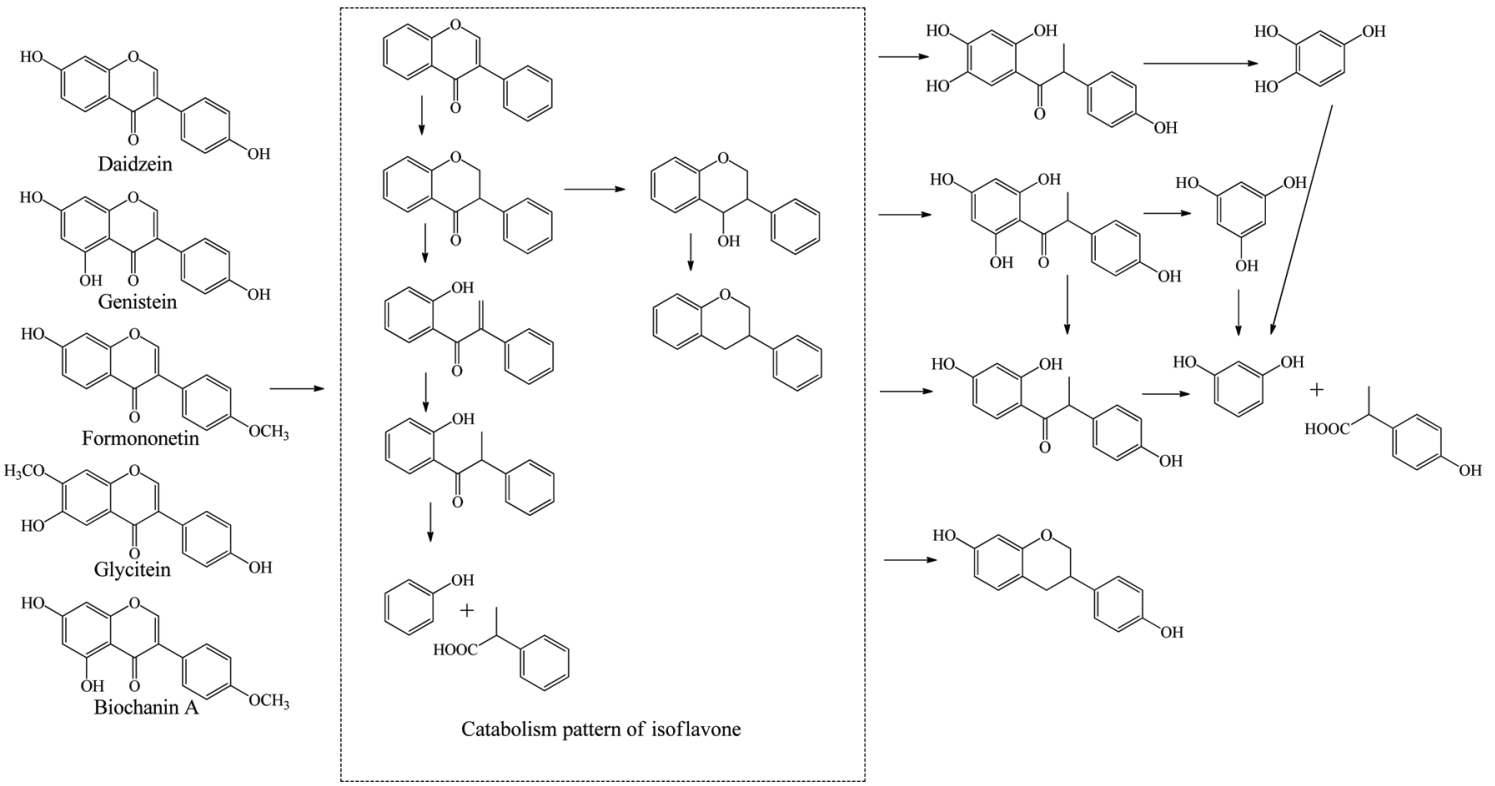

Figure 10. Microbial metablism of isoflavones.

cation, carboxylation, decarboxylation, demethylation, dihydroxylation, and addition reaction. Herein, the most important stage is oxidation, including $\alpha / \beta$-oxidation that usually breaks the $\mathrm{C}-\mathrm{C}$ covalent bonds of the side chain of phenolic acids and the $\mathrm{A} / \mathrm{C}$ ring of flavonoids, leading to the shortening of the side chains with the formation of a carboxyl group (poly-hydroxy cinnamic acid pattern, Fig. 8), as well as the A/C-ring fission (e.g. catechin, isoflavonoids, flavonoids, and anthocyanidins, Fig. 9-12) with production of phenolic acids. Phenolic reduction by gut bacteria includes reductive cleavage of C-ring ether and ketone bonds (e.g., lignans and flavonoids) and hydrogenation of carbon double bonds on either the alkyl side chain (e.g., coumaric acid) or the heterocyclic ring (e.g., isoflavonoids, flavonoids, and lignans, Fig. 10, 11, 13). Moreover, dehydroxylation and decarboxylation are also reduction reactions of which their extent of sensitively depends on specific phenolic structures (resveratrol, polyhydroxybenzoic acid and pol- yhydroxycinnamic acid patterns; Fig. 6, 8, 9) (Bode et al., 2013; Ludwig et al., 2013; Rechner et al., 2004; Rotches-Ribalta et al., 2012b). Hydrolysis occurs at the ester linkage of phenolics such as chlorogenic acid, catechin gallate, and oleuropein which could break the molecules to simpler phenolics and organic acids (Mosele et al., 2015). The central aromatic metabolism (oxidative and reductive fission of phenyl ring) by human gastrointestinal flora is rare and with only few investigations (Heider and Fuchs, 1997; Selma et al., 2009). Currently, even though human gut still lacks a rigorously clarified reaction chain of aromatic degradation similar to those proven in natural soil, water or mine sites and environment, several simple phenolic products of bacterial fermentation such as benzoic acid, pyrogallol, phloroglucinol, and resorcinol are still thought to rapidly go through central aromatic metabolism including aerobic pathway (e.g. gentisic acid, ortho-cleavage, and meta-cleavage pathway) and anaerobic pathway (e.g. benzo-

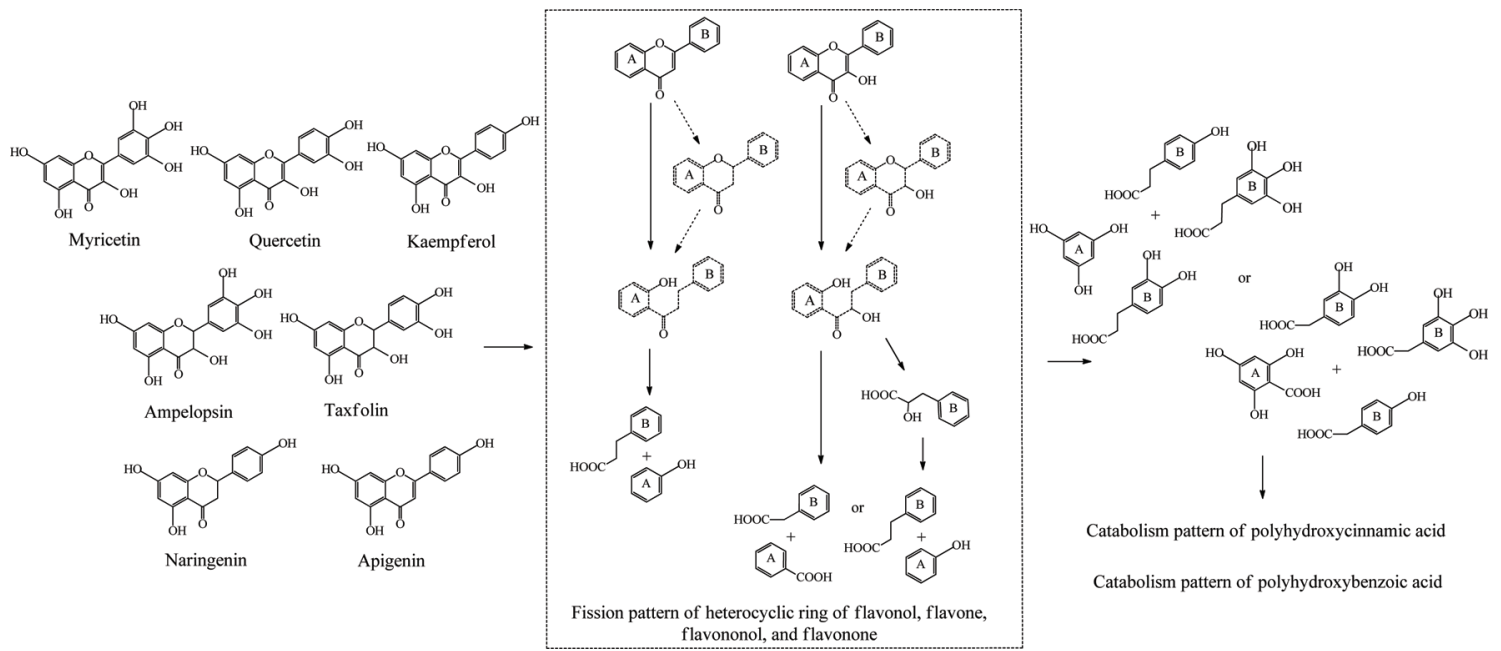

Figure 11. Microbial metablism of flavone, flavonol, flavanone and flavanonol. 


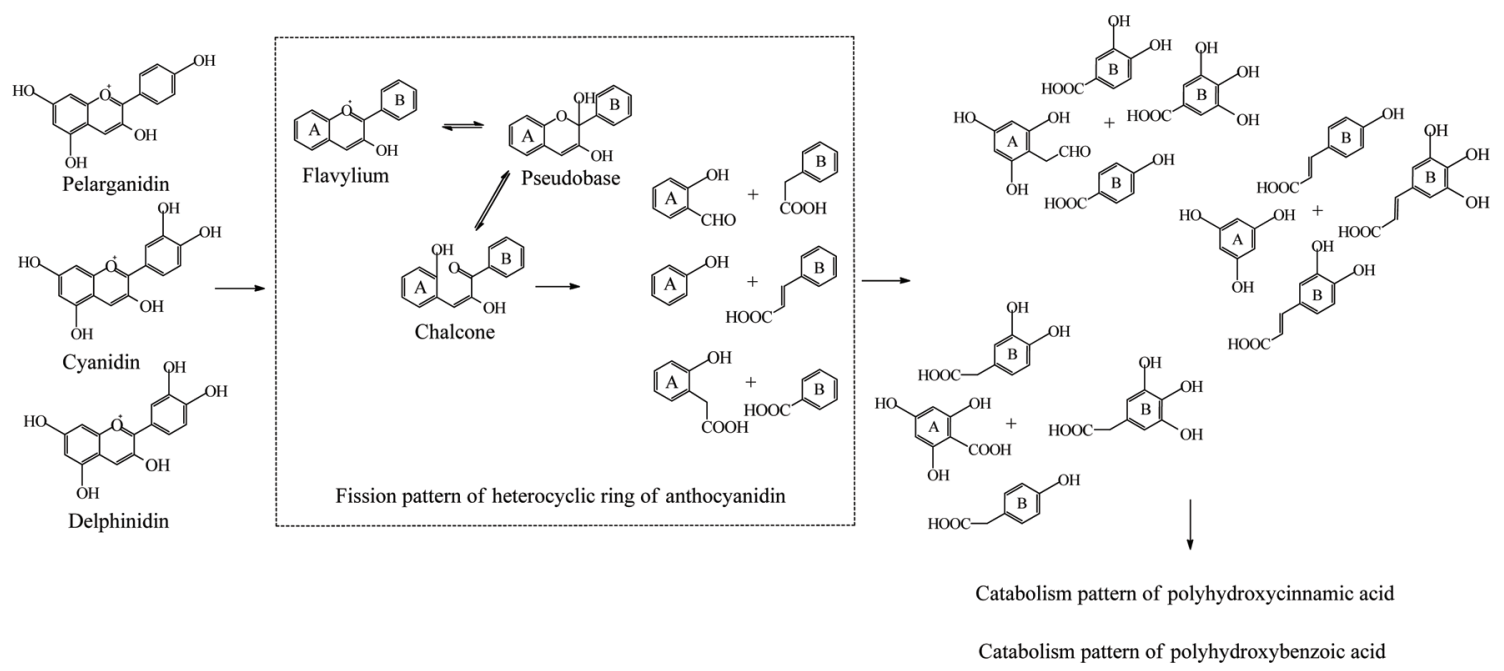

Figure 12. Microbial metablism of anthocyanidin.

yl-CoA pathway), and be degraded into $\mathrm{CO}_{2}$, succinyl-CoA, and acetyl-CoA (e.g. 3 acetyl-CoA and $1 \mathrm{CO}_{2}$ or 2 acetyl-CoA and 0.5 butyryl-CoA) in the gut (Fuchs, 2008; Heider and Fuchs, 1997; Toromanović et al., 2008). As exemplified, the flavonoid A ring metabolite (phloroglucinol) and the fission product of heterocyclic ring of flavonoids, could hardly be detected, indicating its rapid consumption by gut microorganism (Labib et al., 2006). The best known aromatic central degradation has so far been produced by Acidaminococcus fermentuns found in pig and human gut which may enable hydroxybenzoic acid catabolizing to benzoic acid, acetyl group, and $\mathrm{CO}_{2}$. The Eubacterium ramulus and Eubacte- rium oxidoreducens isolated from the human gut are also able to reduce phloroglucinol into 1,3-dioxo-5-hydroxy-cyclohexane and further decompose into acetate and butyrate (Table 16) (Ricaboni et al., 2017; Schneider et al., 1999; Winter et al., 1989). Interestingly, besides the multifarious catabolic reactions described above, an aromatization was observed in the in vitro incubation of quinic and shikimic acids by rat caceal extract, these two simple multihydroxyl cyclohexane carboxylic acids could be converted to protocatechuic acid, then be further catabolized to catechol (Scheline, 1968a).

It is important to emphasize that even though taxonomic and

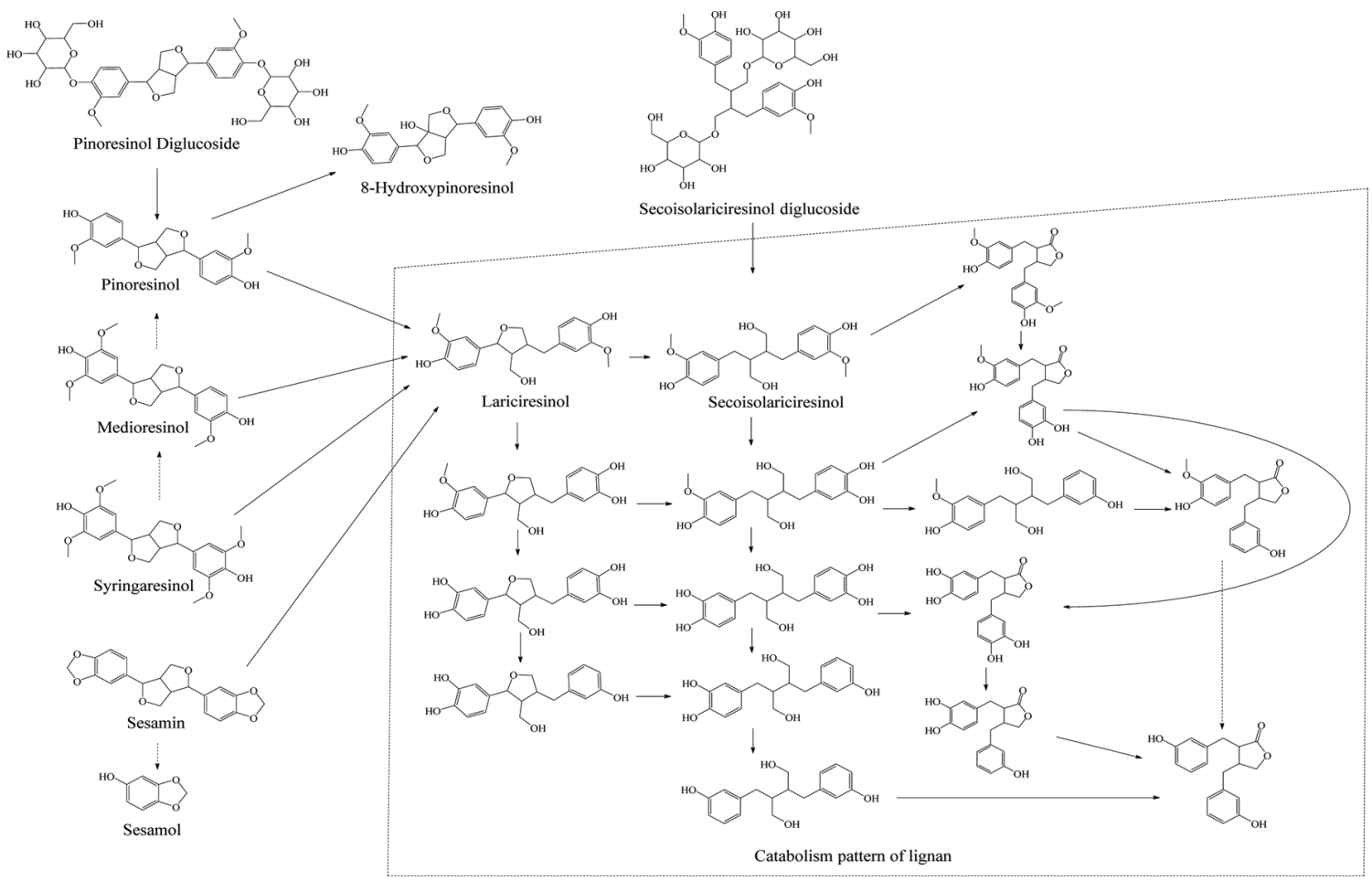

Figure 13. Microbial metablism of lignan. 
Table 17. Catabolism rate of phenolic extracts of nuts-cocoa cream by rat colonic microflora

\begin{tabular}{lllllll}
\hline \multirow{2}{*}{ Substrate } & Substrate concentration in & \multicolumn{5}{c}{ Percentage (\%) remained after incubation, $\mathbf{h}$} \\
\cline { 3 - 6 } & diluted rat fecal inoculum, pM & $\mathbf{1}$ & $\mathbf{2}$ & $\mathbf{4}$ & $\mathbf{2 4}$ & $\mathbf{4 8}$ \\
\hline Rutin & 23.0 & $95.7 \pm 9.1$ & $87.0 \pm 7.4$ & $75.5 \pm 6.1$ & $1.5 \pm 0.1$ & $1.4 \pm 0.1$ \\
Quercetin rhamnoside & 7.3 & $111.0 \pm 10.7$ & $108.2 \pm 10.4$ & $27.4 \pm 2.6$ & $7.7 \pm 0.4$ & $0.8 \pm 0$ \\
Quercetin & 1.0 & $64.0 \pm 3.0$ & $110.0 \pm 11.0$ & $370.0 \pm 31.0$ & $33.0 \pm 2.0$ & 0 \\
Kaempferol-rutinoside & 9.0 & $97.8 \pm 9.7$ & $98.9 \pm 7.2$ & $87.8 \pm 3.6$ & $0.2 \pm 0$ & 0 \\
Naringenin & 13.0 & $100 \pm 0.9$ & $76.9 \pm 7.6$ & $36.2 \pm 2.5$ & $50.8 \pm 4.9$ & 0 \\
Luteolin & 32.0 & $93.8 \pm 9.4$ & $90.6 \pm 6.6$ & $46.9 \pm 4.4$ & $29.7 \pm 2.9$ & $15.6 \pm 1.5$ \\
Gallic acid & 43.0 & $148.8 \pm 14.2$ & $155.8 \pm 14.0$ & $134.9 \pm 1.3$ & $74.4 \pm 7.2$ & $65.1 \pm 5.3$ \\
Protocatechuic acid & 296.0 & $95.9 \pm 8.1$ & $97.3 \pm 5.7$ & $89.5 \pm 7.1$ & $92.2 \pm 8.4$ & $85.8 \pm 7.1$ \\
\hline
\end{tabular}

Adapted from Serra et al. (2012).

quantitative differences exist in individuals, people may possess the same phenolic catabolism capacity in the gut as the bacteria from distinct genera are able to catalyze the same reaction. The genes encoding a core activity from different taxa are called "functional core" and diverse gut microorganism managing the identical reaction from different individuals may be called "core microbiome" (Turnbaugh et al., 2009; Willson and Situ, 2018). Compared with the only two well-known enzymes LPH and cinnamoyl esterase that catalyze de-esterification in the small intestinal lumen, the enzymes for colonic microbial decomposition are more complicated and diverse as shown in Table 14. Consistent with all other enzymatic reaction, distinct substrates with identical core structure catalyzed by the same gut flora render a significantly different reaction rate (Tables 17 and 18). Hence, understanding the phenolic catabolism variability in gut microbiota across various substrates and consumer groups, and correlating it with specific functions of gut catabolites, is going to serve as a reference for consumption of phenolics with healthful effects.

\section{Outlook}

Natural phenolic consumption has been acknowledged as an indispensable factor of a healthy diet. Apart from their well-recognized antioxidant activity, other potential health effects of phenolics such as anti-inflammatory, anti-cancer, antimutagenic, cardio-/ hepato-/neuroprotective, antihypertensive, anti-diabetic, and diuretic effects, as well as inhibition of viral multiplication, blood coagulation, histamine release, and cyclooxygenases activity have also been widely reported (Ferreira et al., 2017; Shahidi and Ambigaipalan, 2015). These natural phenolics are also used as supplements or drugs. Several well-developed phenolic supplements could easily be found in the wholesale and pharmacy outlets including Lipo-Flavonoid ${ }^{\circledR}$ (lemon phenolic extract with Eriodictyol Glycoside), and Daflon (90\% diosmin and 10\% hesperidin). Other prescription drugs such as Veregen ${ }^{\circledR}$ ointment (catechin derivatives) and Mytesi ${ }^{\mathrm{TM}}$ tablets (oligomeric proanthocyanidin mixture) have been confirmed as commercial drugs by US FDA. Beyond natural phenolic products, traditional synthetic phenolics such as BHT (butylated hydroxytoluene), BHA (butylated hydroxyanisole), TBHQ (tertiary-butylhydroquinone), TBMP (2-tert-butyl4-methylphenol), PG (propyl gallate), and aspirin (acetylsalicylic acid) are permitted and employed in food processing and pharmaceutical industries. Meanwhile for the sake of increasing their bioavailability as well as enhancing safety and application range, numerous novel phenolics such as phenolic fatty acid esters and phenolic amino acid esters have been synthesized and further tests on their physiochemical and bioactive properties have been reported (Oh et al., 2018; Shahidi and Ambigaipalan, 2015; Zhong and Shahidi, 2012).

Surely, several works on bioavailability and physiological activity of the prevalent natural or synthetic phenolic standards/mixtures have been carried out but due to the diversity of phenolics and the personal variation, there is still a need for a more clear and thorough understanding of the specific patterns of phenolic bioavailability. Moreover, there are no recommended dietary reference intake (DRI) levels for polyphenolics as no exact physiological evidence exists to verify their nutritional and healthful

Table 18. Flavonoid standards catabolism rate by pig caecal microflora

\begin{tabular}{llllllll}
\hline \multirow{2}{*}{ Substrate } & \multirow{2}{*}{$\begin{array}{l}\text { Substrate concentration in } \\
\text { diluted pig caecal inoculum, } \mathbf{m M}\end{array}$} & \multicolumn{5}{c}{ Percentage (\%) remained after incubation, $\mathbf{h}$} \\
\cline { 3 - 8 } & $\mathbf{2}$ & $\mathbf{4}$ & $\mathbf{6}$ & $\mathbf{8}$ & $\mathbf{1 0}$ & $\mathbf{2 4}$ \\
\hline Galangin & 125 & $95.8 \pm 8.8$ & $83.3 \pm 5.9$ & $54.2 \pm 5.8$ & $19.8 \pm 4.4$ & $7.3 \pm 1.5$ & 0 \\
Kaempferol & 125 & $55.5 \pm 13.7$ & $33.3 \pm 5.9$ & $5.6 \pm 0.9$ & 0 & 0 & 0 \\
Apigenin & 175 & $94.4 \pm 1.9$ & $91.0 \pm 4.9$ & $78.5 \pm 6.9$ & $78.4 \pm 1.0$ & $67.4 \pm 1.0$ & $59.0 \pm 0.9$ \\
Luteolin & 175 & $95.8 \pm 1.9$ & $81.6 \pm 1.9$ & $86.1 \pm 3.9$ & $82.6 \pm 4.9$ & $65.3 \pm 7.9$ & $56.2 \pm 4.9$ \\
Quercetin & 125 & $53.7 \pm 1.5$ & $38.9 \pm 1.5$ & $18.9 \pm 3.0$ & $5.3 \pm 1.5$ & 0 & 0 \\
Chrysin & 175 & $100.0 \pm 3.9$ & $99.9 \pm 6.0$ & $98.6 \pm 9.9$ & $100.7 \pm 1.0$ & $98.6 \pm 4.0$ & $99.9 \pm 5.9$ \\
\hline
\end{tabular}

Adapted from Labib et al. (2006). 
qualification, so phenolic supplements cannot even legally be labeled with either antioxidant capacity or any other nutritional/ functional claims in the US and many other countries. As well, standardization and legislation of phenolic-rich herbal and pharmaceutical products are also lacking consensus and compatibility in different regions/countries such as EU, US, Japan, South Korea, and China. Various natural/artificial phenolic nutraceutical, pharmaceutical and food additive products are expected to be verified, applied and approved. More efforts are also in progress, thus a number of bioavailability studies covering phenolic digestion, absorption, transport, metabolism, colonic catabolism, excretion, and physiological effects are still in need of further clarification and elaboration.

\section{References}

21-CFR-314.3. (2016). "Application for FDA Approval to Market a New Drug [21CFR314.3]," Internet Available: "Application for FDA Approval to Market a New Drug [21CFR314.3]," Internet Available: https:// www.ecfr.gov/cgi-bin/text-idx?SID=df102bf2c65c682588f69d4133a $4 d 145 \& m c=t r u e \& n o d e=p t 21.5 .314 \&$ rgn $=$ div5\#se21.5.314 13. Page Last Updated: Feb. 1, 2018, 1_13. Page Last Updated: Feb. 1, 2018, 1.

Abdel-Aal, E.S. (2008). Effects of baking on protein digestibility of organic spelt products determined by two in vitro digestion methods. LWTFood Sci. Technol. 41(7): 1282-1288.

Acosta-Estrada, B.A., Gutiérrez-Uribe, J.A., and Serna-Saldívar, S.O. (2014). Bound phenolics in foods, a review. Food Chem. 52: 46-55.

Alvarez-Sala, A., Garcia-Llatas, G., Cilla, A., Barberá, R., Sánchez-Siles, L.M., and Lagarda, M.A.J.S. (2016). Impact of lipid components and emulsifiers on plant sterols bioaccessibility from milk-based fruit beverages. J. Agric. Food Chem. 64(28): 5686-5691.

Amarowicz, R., and Pegg, R.B. (2008). Legumes as a source of natural antioxidants. Eur. J. Lipid Sci. Technol. 110(10): 865-878.

An, C.-Y., Sun, Z.-Z., Shen, L., and Ji, H.-F. (2017). Biotransformation of food spice curcumin by gut bacterium Bacillus megaterium DCMB-002 and its pharmacological implications. Food Nutr. Res. 61(1): 1412814.

Anderson, J.M. (2001). Molecular structure of tight junctions and their role in epithelial transport. Physiology 16(3): 126-130.

Andlauer, W., Kolb, J., Stehle, P., and Fürst, P. (2000). Absorption and metabolism of genistein in isolated rat small intestine. J. Nutr. 130(4): 843-846.

Andreasen, M.F., Kroon, P.A., Williamson, G., and Garcia-Conesa, M.-T. (2001a). Esterase activity able to hydrolyze dietary antioxidant hydroxycinnamates is distributed along the intestine of mammals. J. Agric. Food Chem. 49(11): 5679-5684.

Andreasen, M.F., Kroon, P.A., Williamson, G., and Garcia-Conesa, M.-T. (2001b). Intestinal release and uptake of phenolic antioxidant diferulic acids. Free Radical Biol. Med. 31(3): 304-314.

Andries, M.J., Lucier, G.W., Goldstein, J., and Thompson, C.L. (1990). Involvement of cytochrome P-450c in alpha-naphthoflavone metabolism by rat liver microsomes. Mol. Pharmacol. 37(6): 990-995.

Anson, N.M., Selinheimo, E., Havenaar, R., Aura, A.-M., Mattila, I., Lehtinen, P., Bast, A., Poutanen, K., and Haenen, G.R. (2009). Bioprocessing of wheat bran improves in vitro bioaccessibility and colonic metabolism of phenolic compounds. J. Agric. Food Chem. 57(14): 6148-6155.

Appeldoorn, M.M., Vincken, J.-P., Gruppen, H., and Hollman, P.C. (2009). Procyanidin dimers $\mathrm{A} 1, \mathrm{~A} 2$, and $\mathrm{B} 2$ are absorbed without conjugation or methylation from the small intestine of rats. J. Nutr. 139(8): 1469-1473.

Aragonès, G., Danesi, F., Del Rio, D., and Mena, P. (2017). The importance of studying cell metabolism when testing the bioactivity of phenolic compounds. Trends Food Sci. Technol. 69: 230-242.

Arenas, E.H., and Trinidad, T.P. (2017). Fate of polyphenols in pili (Canarium ovatum Engl.) pomace after in vitro simulated digestion. Asian Pac. J. Trop. Biomed. 7(1): 53-58.

Ashong, J., Muthayya, S., De-Regil, L.M., Laillou, A., Guyondet, C., MoenchPfanner, R., Burford, B.J., and Peña-Rosas, J.P. (2012). Fortification of rice with vitamins and minerals for addressing micronutrient malnu- trition. Cochrane Database Syst Rev. (6).

Astorga, B., Ekins, S., Morales, M., and Wright, S.H. (2012). Molecular determinants of ligand selectivity for the human multidrug and toxin extruder proteins MATE1 and MATE2-K. J. Pharmacol. Exp. Ther. 341(3): 743-755.

Aura, A.-M. (2008). Microbial metabolism of dietary phenolic compounds in the colon. Phytochem. Rev. 7(3): 407-429.

Aura, A.-M., Martin-Lopez, P., O'Leary, K.A., Williamson, G., Oksman-Caldentey, K.-M., Poutanen, K., and Santos-Buelga, C. (2005). In vitro metabolism of anthocyanins by human gut microflora. Eur. J. Nutr. 44(3): 133-142.

Aura, A.-M., O'leary, K., Williamson, G., Ojala, M., Bailey, M., PuupponenPimiä, R., Nuutila, A., Oksman-Caldentey, K.-M., and Poutanen, K. (2002). Quercetin derivatives are deconjugated and converted to hydroxyphenylacetic acids but not methylated by human fecal flora in vitro. J. Agric. Food Chem. 50(6): 1725-1730.

Ávila, M., Hidalgo, M., Sánchez-Moreno, C., Pelaez, C., Requena, T., and de Pascual-Teresa, S. (2009). Bioconversion of anthocyanin glycosides by Bifidobacteria and Lactobacillus. Food Res. Int. 42(10): 1453-1461.

Aydin, E., and Gocmen, D. (2015). The influences of drying method and metabisulfite pre-treatment on the color, functional properties and phenolic acids contents and bioaccessibility of pumpkin flour. LWTFood Sci. Technol. 60(1): 385-392.

Barbier, O., Turgeon, D., Girard, C., Green, M.D., Tephly, T.R., Hum, D.W., and Bélanger, A. (2000). 3'-azido-3'-deoxythimidine (AZT) is glucuronidated by human UDP-glucuronosyltransferase 2B7 (UGT2B7). Drug Metab. Dispos. 28(5): 497-502.

Barthelmebs, L., Divies, C., and Cavin, J.-F. (2000). Knockout of the p-coumarate decarboxylase gene from Lactobacillus plantarum reveals the existence of two other inducible enzymatic activities involved in phenolic acid metabolism. Appl. Environ. Microbiol. 66(8): 3368-3375.

Berg, R.D. (1996). The indigenous gastrointestinal microflora. Trends Microbiol. 4(11): 430-435.

Bering, S., Bukhave, K., Henriksen, M., Sandström, B., Pariagh, S., Fairweather-Tait, S.J., and Lund, E.K. (2006). Development of a three-tier in vitro system, using Caco-2 cells, to assess the effects of lactate on iron uptake and transport from rye bread following in vitro digestion. J. Sci. Food Agric. 86(14): 2438-2444.

Bermúdez-Soto, M.-J., Tomás-Barberán, F.-A., and García-Conesa, M.-T. (2007). Stability of polyphenols in chokeberry (Aronia melanocarpa) subjected to in vitro gastric and pancreatic digestion. Food Chem. 102(3): 865-874.

Biesalski, H.-K., Dragsted, L.O., Elmadfa, I., Grossklaus, R., Müller, M., Schrenk, D., Walter, P., and Weber, P. (2009). Bioactive compounds: definition and assessment of activity. Nutrition 25(11-12): 12021205

Bik, E.M., Eckburg, P.B., Gill, S.R., Nelson, K.E., Purdom, E.A., Francois, F., Perez-Perez, G., Blaser, M.J., and Relman, D.A. (2006). Molecular analysis of the bacterial microbiota in the human stomach. Proc. Natl. Acad. Sci. 103(3): 732-737.

Blaut, M., and Clavel, T. (2007). Metabolic diversity of the intestinal microbiota: implications for health and disease. J. Nutr. 137(3): 751S-755S.

Bloem, A., Bertrand, A., Lonvaud-Funel, A., and De Revel, G. (2007). Vanillin production from simple phenols by wine-associated lactic acid bacteria. Lett. Appl. Microbiol. 44(1): 62-67.

Blok, J., Mulder-Stapel, A., Ginsel, L., and Daems, W.T. (1981). Endocytosis in absorptive cells of cultured human small-intestinal tissue: horseradish peroxidase, lactoperoxidase, and ferritin as markers. Cell Tissue Res. 216(1): 1-13.

Bode, L.M., Bunzel, D., Huch, M., Cho, G.-S., Ruhland, D., Bunzel, M., Bub, A., Franz, C.M., and Kulling, S.E. (2013). In vivo and in vitro metabolism of trans-resveratrol by human gut microbiota. Am. J. Clin. Nutr. 97(2): 295-309.

Booijink, C.C., Zoetendal, E.G., Kleerebezem, M., and De Vos, W.M. (2007). Microbial communities in the human small intestine: coupling diversity to metagenomics. Future Microbiol. 2: 285-295.

Booth, A.N., Murray, C.W., Jones, F.T., and DeEds, F. (1956). The metabolic fate of rutin and quercetin in the animal body. J. Biol. Chem. 223(1): 251-257.

Borst, P., Evers, R., Kool, M., and Wijnholds, J. (2000). A family of drug transporters: the multidrug resistance-associated proteins. J. Natl. 
Cancer Inst. 92(16): 1295-1302

Bouayed, J., Hoffmann, L., and Bohn, T. (2011). Total phenolics, flavonoids, anthocyanins and antioxidant activity following simulated gastro-intestinal digestion and dialysis of apple varieties: Bioaccessibility and potential uptake. Food Chem. 128(1): 14-21.

Boyer, J., Brown, D., and Liu, R.H. (2005). In vitro digestion and lactase treatment influence uptake of quercetin and quercetin glucoside by the Caco- 2 cell monolayer. Nutrition 4(1): 1 .

Braune, A., and Blaut, M. (2016). Bacterial species involved in the conversion of dietary flavonoids in the human gut. Gut Microbes $7(3)$ 216-234.

Braune, A., Engst, W., and Blaut, M. (2005). Degradation of neohesperidin dihydrochalcone by human intestinal bacteria. J. Agric. Food Chem. 53(5): 1782-1790.

Brown, E.M., Nitecki, S., Pereira-Caro, G., McDougall, G.J., Stewart, D., Rowland, I., Crozier, A., and Gill, C.I. (2014). Comparison of in vivo and in vitro digestion on polyphenol composition in lingonberries: Potential impact on colonic health. Biofactors 40(6): 611-623.

Buchanan, C.J., Wallace, G., Fry, S.C., and Eastwood, M.A. (1996). InVivo Release of 14C-Labelled Phenolic Groups from Intact Dietary Spinach Cell Walls During Passage Through the Rat Intestine. J. Sci. Food Agric. 71(4): 459-469.

Bugianesi, R., Salucci, M., Leonardi, C., Ferracane, R., Catasta, G., Azzini, E., and Maiani, G. (2004). Effect of domestic cooking on human bioavailability of naringenin, chlorogenic acid, lycopene and $\beta$-carotene in cherry tomatoes. Eur. J. Nutr. 43(6): 360-366.

Burton, K., and Smith, M. (1977). Endocytosis and immunoglobulin transport across the small intestine of the new-born pig. J. Physiol. 270(2): 473-488.

Cai, H., Boocock, D.J., Steward, W.P., and Gescher, A.J. (2007). Tissue distribution in mice and metabolism in murine and human liver of apigenin and tricin, flavones with putative cancer chemopreventive properties. Cancer Chemother. Pharmacol. 60(2): 257-266.

Calamini, B., Ratia, K., Malkowski, M.G., Cuendet, M., Pezzuto, J.M., Santarsiero, B.D., and Mesecar, A.D. (2010). Pleiotropic mechanisms facilitated by resveratrol and its metabolites. Biochem. J. 429(2): 273-282.

Camilleri, M., Colemont, L., Phillips, S.F., Brown, M.L., Thomforde, G.M. Chapman, N., and Zinsmeister, A.R. (1989). Human gastric emptying and colonic filling of solids characterized by a new method. Am. J. Physiol. Gastrointest. Liver Physiol. 257(2): G284-G290.

Canivenc-Lavier, M.-C., Brunold, C., Siess, M.-H., and Suschetet, M. (1993). Evidence for tangeretin $\mathrm{O}$-demethylation by rat and human liver microsomes. Xenobiotica 23(3): 259-266.

Carbonaro, M., Grant, G., and Pusztai, A. (2001). Evaluation of polyphenol bioavailability in rat small intestine. Eur. J. Nutr. 40(2): 84-90.

Carbonell-Capella, J.M., Buniowska, M., Barba, F.J., Esteve, M.J., and Frígola, A. (2014). Analytical methods for determining bioavailability and bioaccessibility of bioactive compounds from fruits and vegetables: A review. Compr. Rev. Food Sci. Food Saf. 13(2): 155-171.

Cermak, R., Landgraf, S., and Wolffram, S. (2004). Quercetin glucosides inhibit glucose uptake into brush-border-membrane vesicles of porcine jejunum. Br. J. Nutr. 91(6): 849-855.

Chandrasekara, A., and Shahidi, F. (2012). Bioaccessibility and antioxidant potential of millet grain phenolics as affected by simulated in vitro digestion and microbial fermentation. J. Funct. Food 4(1): 226-237.

Chen, C.-H., Hsu, H.-J., Huang, Y.-J., and Lin, C.-J. (2007). Interaction of flavonoids and intestinal facilitated glucose transporters. Planta Med. 73(04): 348-354.

Chen, G.-L., Chen, S.-G., Xie, Y.-Q., Chen, F., Zhao, Y.-Y., Luo, C.-X., and Gao, Y.-Q. (2015). Total phenolic, flavonoid and antioxidant activity of 23 edible flowers subjected to in vitro digestion. J. Funct. Food 17: 243-259.

Chen, G.-L., Hu, K., Zhong, N.-J., Guo, J., Gong, Y.-S., Deng, X.-T., Huang, Y. S., Chu, D.-K., and Gao, Y.-Q. (2013). Antioxidant capacities and total polyphenol content of nine commercially available tea juices measured by an in vitro digestion model. Eur. Food Res. Technol. 236(2): 303-310.

Chen, J., Lin, H., and Hu, M. (2005). Absorption and metabolism of genistein and its five isoflavone analogs in the human intestinal Caco-2 model. Cancer Chemother. Pharmacol. 55(2): 159-169.
Chen, Y., Li, Q., Zhao, T., Zhang, Z., Mao, G., Feng, W., Wu, X., and Yang, L. (2017). Biotransformation and metabolism of three mulberry anthocyanin monomers by rat gut microflora. Food Chem. 237: 887-894.

Chen, Z., Shi, T., Zhang, L., Zhu, P., Deng, M., Huang, C., Hu, T., Jiang, L., and $\mathrm{Li}$, J. (2016). Mammalian drug efflux transporters of the ATP binding cassette $(A B C)$ family in multidrug resistance: $A$ review of the past decade. Cancer Lett. 370(1): 153-164.

Chen, Z., Zheng, S., Li, L., and Jiang, H. (2014). Metabolism of flavonoids in human: a comprehensive review. Curr. Drug Metab. 15(1): 48-61.

Cheng, J.-R., Liu, X.-M., Chen, Z.-Y., Zhang, Y.-S., and Zhang, Y.-H. (2016) Mulberry anthocyanin biotransformation by intestinal probiotics. Food Chem. 213: 721-727.

Cheng, K.-J., Jones, G., Simpson, F., and Bryant, M. (1969). Isolation and identification of rumen bacteria capable of anaerobic rutin degradation. Can. J. Microbiol. 15(12): 1365-1371.

Cheng, K.-J., Krishnamurty, H., Jones, G., and Simpson, F. (1971). Identification of products produced by the anaerobic degradation of naringin by Butyrivibrio sp. C3. Can. J. Microbiol. 17(1): 129-131.

Cheng, Z., Liu, H., Yu, N., Wang, F., An, G., Xu, Y., Liu, Q., Guan, C.-B., and Ayrton, A. (2012). Hydrophilic anti-migraine triptans are substrates for OATP1A2, a transporter expressed at human blood-brain barrier. Xenobiotica 42(9): 880-890.

Cheng, Z., Radominska-Pandya, A., and Tephly, T.R. (1998). Cloning and expression of human UDP-glucuronosyltransferase (UGT) 1A8. Arch. Biochem. Biophys. 356(2): 301-305.

Cheng, Z., Radominska-Pandya, A., and Tephly, T.R. (1999). Studies on the substrate specificity of human intestinal UDP-glucuronosyltransferases $1 \mathrm{~A} 8$ and 1A10. Drug Metab. Dispos. 27(10): 1165-1170.

Chiang, C.-J., Kadouh, H., and Zhou, K. (2013). Phenolic compounds and antioxidant properties of gooseberry as affected by in vitro digestion. LWT-Food Sci. Technol. 51(2): 417-422.

Chimalakonda, K.C., Bratton, S.M., Le, V.-H., Yiew, K.H., Dineva, A., Moran, C.L., James, L.P., Moran, J.H., and Radominska-Pandya, A. (2011) Conjugation of synthetic cannabinoids JWH-018 and JWH-073, metabolites by human UDP-glucuronosyltransferases. Drug Metab. Dispos. 39(10): 1967-1976.

Chiou, Y.-S., Wu, J.-C., Huang, Q., Shahidi, F., Wang, Y.-J., Ho, C.-T., and Pan, M.-H. (2014). Metabolic and colonic microbiota transformation may enhance the bioactivities of dietary polyphenols. J. Funct. Food 7 : 3-25.

Clavel, T., Henderson, G., Engst, W., Doré, J., and Blaut, M. (2006). Phylogeny of human intestinal bacteria that activate the dietary lignan secoisolariciresinol diglucoside. FEMS Microbiol. Ecol. 55(3): 471-478.

Collins, A.C., Cashaw, J.L., and Davis, V.E. (1973). Dopamine-derived tetrahydroisoquinoline alkaloids-inhibitors of neuroamine metabolism. Biochem. Pharmacol. 22(18): 2337-2348.

Contreras, M., Borrás-Linares, I., Herranz-López, M., Micol, V., and SeguraCarretero, A. (2016). Further exploring the absorption and enterocyte metabolism of quercetin forms in the Caco-2 model using nanoLC-TOF-MS. Electrophoresis 37(7-8): 998-1006.

Correa-Betanzo, J., Allen-Vercoe, E., McDonald, J., Schroeter, K., Corredig, M., and Paliyath, G. (2014). Stability and biological activity of wild blueberry (Vaccinium angustifolium) polyphenols during simulated in vitro gastrointestinal digestion. Food Chem. 165: 522-531.

Corrêa, R.C., Haminiuk, C.W., Barros, L., Dias, M.I., Calhelha, R.C., Kato, C.G., Correa, V.G., Peralta, R.M., and Ferreira, I.C. (2017). Stability and biological activity of Merlot (Vitis vinifera) grape pomace phy tochemicals after simulated in vitro gastrointestinal digestion and colonic fermentation. J. Funct. Food 36: 410-417.

Costello, E.K., Lauber, C.L., Hamady, M., Fierer, N., Gordon, J.I., and Knight, R. (2009). Bacterial community variation in human body habitats across space and time. Science 326(5960): 1694-1697.

Cotillard, A., Kennedy, S.P., Kong, L.C., Prifti, E., Pons, N., Le Chatelier, E., Almeida, M., Quinquis, B., Levenez, F., and Galleron, N. (2013). Dietary intervention impact on gut microbial gene richness. Nature 500(7464): 585

Court, M.H., Zhang, X., Ding, X., Yee, K.K., Hesse, L.M., and Finel, M (2012). Quantitative distribution of mRNAs encoding the 19 human UDP-glucuronosyltransferase enzymes in 26 adult and 3 fetal tissues. Xenobiotica 42(3): 266-277.

Crespy, V., Morand, C., Besson, C., Manach, C., Demigne, C., and Remesy, 
C. (2002). Quercetin, but not its glycosides, is absorbed from the rat stomach. J. Agric. Food Chem. 50(3): 618-621.

Crespy, V., Morand, C., Manach, C., Besson, C., Demigne, C., and Remesy, C. (1999). Part of quercetin absorbed in the small intestine is conjugated and further secreted in the intestinal lumen. Am. J. Physiol. Gastrointest. Liver Physiol. 277(1): G120-G126.

Cui, D., and Morris, M.E. (2009). The drug of abuse $\psi$-hydroxybutyrate is a substrate for sodium-coupled monocarboxylate transporter (SMCT) 1 (SLC5A8): characterization of SMCT-mediated uptake and inhibition. Drug Metab. Dispos. 37(7): 1404-1410.

Cunningham, P., Afzal-Ahmed, I., and Naftalin, R.J. (2006). Docking studies show that D-glucose and quercetin slide through the transporter GLUT1. J. Biol. Chem. 281(9): 5797-5803.

D'Antuono, I., Garbetta, A., Linsalata, V., Minervini, F., and Cardinali, A. (2015). Polyphenols from artichoke heads (Cynara cardunculus (L.) subsp. scolymus Hayek): In vitro bio-accessibility, intestinal uptake and bioavailability. Food Funct. 6(4): 1268-1277.

Day, A.J., Bao, Y., Morgan, M.R., and Williamson, G. (2000a). Conjugation position of quercetin glucuronides and effect on biological activity. Free Radical Biol. Med. 29(12): 1234-1243.

Day, A.J., Cañada, F.J., Díaz, J.C., Kroon, P.A., Mclauchlan, R., Faulds, C.B., Plumb, G.W., Morgan, M.R., and Williamson, G. (2000b). Dietary flavonoid and isoflavone glycosides are hydrolysed by the lactase site of lactase phlorizin hydrolase. FEBS Lett. 468(2-3): 166-170.

Day, A.J., DuPont, M.S., Ridley, S., Rhodes, M., Rhodes, M.J., Morgan, M.R., and Williamson, G. (1998). Deglycosylation of flavonoid and isoflavonoid glycosides by human small intestine and liver $\beta$-glucosidase activity. FEBS Lett. 436(1): 71-75.

Day, A.J., Gee, J.M., DuPont, M.S., Johnson, I.T., and Williamson, G. (2003). Absorption of quercetin-3-glucoside and quercetin- 4 '-glucoside in the rat small intestine: the role of lactase phlorizin hydrolase and the sodium-dependent glucose transporter. Biochem. Pharmacol. 65(7): 1199-1206.

De Boever, P., Deplancke, B., and Verstraete, W. (2000). Fermentation by gut microbiota cultured in a simulator of the human intestinal microbial ecosystem is improved by supplementing a soygerm powder. J. Nutr. 130(10): 2599-2606.

De Montellano, P.R.O. (2005). Cytochrome P450: structure, mechanism, and biochemistry, Springer Science \& Business Media.

Déprez, S., Brezillon, C., Rabot, S., Philippe, C., Mila, I., Lapierre, C., and Scalbert, A. (2000). Polymeric proanthocyanidins are catabolized by human colonic microflora into low-molecular-weight phenolic acids. J. Nutr. 130(11): 2733-2738.

Deprez, S., Mila, I., Huneau, J.-F., Tome, D., and Scalbert, A. (2001). Transport of proanthocyanidin dimer, trimer, and polymer across monolayers of human intestinal epithelial Caco-2 cells. Antioxid. Redox. Signal. 3(6): 957-967.

Déprez, S.P., Brezillon, C., Rabot, S., Philippe, C., Mila, I., Lapierre, C., and Scalbert, A. (2000). Polymeric proanthocyanidins are catabolized by human colonic microflora into low-molecular-weight phenolic acids. J. Nutr. 130(11): 2733-2738.

Dethlefsen, L., Eckburg, P.B., Bik, E.M., and Relman, D.A. (2006). Assembly of the human intestinal microbiota. Trends Ecol. Evol. 21(9): 517-523.

Dewhirst, F.E., Chen, T., Izard, J., Paster, B.J., Tanner, A.C., Yu, W.-H., Lakshmanan, A., and Wade, W.G. (2010). The human oral microbiome. J. Bacteriol. 192(19): 5002-5017.

Dhananjay, P., Krishna, V.R., Dutt, V.A., and Mitra, A.K. (2013). Biology of ocular transporters: efflux and influx transporters in the eye Ocular Transporters and Receptors Elsevier, pp. 37-84.

Domínguez-Avila, J.A., Wall-Medrano, A., Velderrain-Rodríguez, G.R., Chen, C.-Y.O., Salazar-López, N.J., Robles-Sánchez, M., and GonzálezAguilar, G.A. (2017). Gastrointestinal interactions, absorption, splanchnic metabolism and pharmacokinetics of orally ingested phenolic compounds. Food Funct. 8(1): 15-38.

Dong, L., Yin, J., Zhao, J., Ma, S.-R., Wang, H.-R., Wang, M., Chen, W., and Wei, W.-Q. (2018). Microbial similarity and preference for specific sites in healthy oral cavity and esophagus. Front Microbiol 9.

Donovan, J.L., Crespy, V., Manach, C., Morand, C., Besson, C., Scalbert, A., and Rémésy, C. (2001). Catechin is metabolized by both the small intestine and liver of rats. J. Nutr. 131(6): 1753-1757.

Estudante, M., Morais, J.G., Soveral, G., and Benet, L.Z. (2013). Intestinal drug transporters: an overview. Adv. Drug Deliv. Rev. 65(10): 13401356.

Etcheverry, P., Grusak, M.A., and Fleige, L.E. (2012). Application of in vitro bioaccessibility and bioavailability methods for calcium, carotenoids, folate, iron, magnesium, polyphenols, zinc, and vitamins B6, B12, D, and E. Front Physiol 3: 317.

Faller, A.L.K., Fialho, E., and Liu, R.H. (2012). Cellular antioxidant activity of Feijoada whole meal coupled with an in vitro digestion. J. Agric. Food Chem. 60(19): 4826-4832.

Faria, A., Mateus, N., and Calhau, C. (2012). Flavonoid transport across blood-brain barrier: Implication for their direct neuroprotective actions. Nutr. Aging 1(2): 89-97.

Faria, A., Pestana, D., Azevedo, J., Martel, F., de Freitas, V., Azevedo, I., Mateus, N., and Calhau, C. (2009). Absorption of anthocyanins through intestinal epithelial cells-Putative involvement of GLUT2. Mol. Nutr. Food Res. 53(11): 1430-1437.

Fernandes, I., Faria, A., Calhau, C., de Freitas, V., and Mateus, N. (2014). Bioavailability of anthocyanins and derivatives. J. Funct. Food 7: 54-66.

Ferreira, I.C., Martins, N., and Barros, L. (2017). Phenolic Compounds and Its Bioavailability: In Vitro Bioactive Compounds or Health Promoters? Adv. Food Nutr. Res. 82: 1-44.

Finel, M., Li, X., Gardner-Stephen, D., Bratton, S., Mackenzie, P.I., and Radominska-Pandya, A. (2005). Human UDP-glucuronosyltransferase 1A5: identification, expression, and activity. J. Pharmacol. Exp. Ther. 315(3): 1143-1149.

Fisher, M.B., Paine, M.F., Strelevitz, T.J., and Wrighton, S.A. (2001). The role of hepatic and extrahepatic UDP-glucuronosyltransferases in human drug metabolism. Drug Metab. Rev. 33(3-4): 273-297.

Flores, G., del Castillo, M.L.R., Costabile, A., Klee, A., Guergoletto, K.B., and Gibson, G.R. (2015). In vitro fermentation of anthocyanins encapsulated with cyclodextrins: Release, metabolism and influence on gut microbiota growth. J. Funct. Food 16: 50-57.

Forester, S.C., and Waterhouse, A.L. (2010). Gut metabolites of anthocyanins, gallic acid 3-0-methylgallic acid, and 2, 4, 6-trihydroxybenzaldehyde, inhibit cell proliferation of Caco-2 cells. J. Agric. Food Chem. 58(9): 5320-5327.

Freitas, H.S., Schaan, B.D.A., Seraphim, P.M., Nunes, M.T., and Machado, U.F. (2005). Acute and short-term insulin-induced molecular adaptations of GLUT2 gene expression in the renal cortex of diabetic rats. Mol. Cell. Endocrinol. 237(1-2): 49-57.

Fuchs, G. (2008). Anaerobic metabolism of aromatic compounds. Ann. N. Y. Acad. Sci. 1125(1): 82-99.

Fujio-Vejar, S., Vasquez, Y., Morales, P., Magne, F., Vera-Wolf, P., Ugalde, J.A., Navarrete, P., and Gotteland, M. (2017). The gut microbiota of healthy chilean subjects reveals a high abundance of the phylum verrucomicrobia. Front Microbiol. 8: 1221

Gakh, A.A., Anisimova, N.Y., Kiselevsky, M.V., Sadovnikov, S.V., Stankov, I.N., Yudin, M.V., Rufanov, K.A., Krasavin, M.Y., and Sosnov, A.V. (2010). Dihydro-resveratrol-a potent dietary polyphenol. Bioorg. Med. Chem. Lett. 20(20): 6149-6151.

Galati, G., and O'brien, P.J. (2004). Potential toxicity of flavonoids and other dietary phenolics: significance for their chemopreventive and anticancer properties. Free Radical Biol. Med. 37(3): 287-303.

Galetin, A., Gertz, M., and Houston, J.B. (2010). Contribution of intestinal cytochrome p450-mediated metabolism to drug-drug inhibition and induction interactions. Drug Metab. Pharmacokinet. 25(1): 28-47.

Galijatovic, A., Otake, Y., Walle, U., and Walle, T. (1999). Extensive metabolism of the flavonoid chrysin by human Caco-2 and Hep G2 cells. Xenobiotica 29(12): 1241-1256.

Gao, B., Hagenbuch, B., Kullak-Ublick, G.A., Benke, D., Aguzzi, A., and Meier, P.J. (2000). Organic anion-transporting polypeptides mediate transport of opioid peptides across blood-brain barrier. J. Pharmacol. Exp. Ther. 294(1): 73-79.

Garrett, D.A., Failla, M.L., and Sarama, R.J. (1999). Development of an in vitro digestion method to assess carotenoid bioavailability from meals. J. Agric. Food Chem. 47(10): 4301-4309.

Garrett, W.S., Gordon, J.I., and Glimcher, L.H. (2010). Homeostasis and inflammation in the intestine. Cell 140(6): 859-870.

Gawlik-Dziki, U., Dziki, D., Baraniak, B., and Lin, R. (2009). The effect of simulated digestion in vitro on bioactivity of wheat bread with Tartary buckwheat flavones addition. LWT-Food Sci. Technol. 42(1): 
137-143.

Gaya, P., Arqués, J.L., Medina, M., Álvarez, I., and Landete, J.M. (2016). A new HPLC-PAD/HPLC-ESI-MS method for the analysis of phytoestrogens produced by bacterial metabolism. Food Anal. Methods 9(2): 537-547.

Gee, J.M., DuPont, M.S., Day, A.J., Plumb, G.W., Williamson, G., and Johnson, I.T. (2000). Intestinal transport of quercetin glycosides in rats involves both deglycosylation and interaction with the hexose transport pathway. J. Nutr. 130(11): 2765-2771.

Geier, E.G., Chen, E.C., Webb, A., Papp, A.C., Yee, S.W., Sadee, W., and Giacomini, K.M. (2013). Profiling solute carrier transporters in the human blood-brain barrier. Clin. Pharmacol. Ther. 94(6): 636-639.

Ghosh, C., Hossain, M., Puvenna, V., Martinez-Gonzalez, J., Alexopolous, A., Janigro, D., and Marchi, N. (2013). Expression and functional relevance of UGT 1 A 4 in a cohort of human drug-resistant epileptic brains. Epilepsia 54(9): 1562-1570.

Gibbons, R.J., and Houte, J. (1975). Bacterial adherence in oral microbial ecology. Annu. Rev. Microbiol. 29(1): 19-42.

Gil-Izquierdo, A., Gil, M.I., Ferreres, F., and Tomás-Barberán, F.A. (2001). In vitro availability of flavonoids and other phenolics in orange juice. J. Agric. Food Chem. 49(2): 1035-1041.

Gil-Izquierdo, A., Zafrilla, P., and Tomás-Barberán, F.A. (2002). An in vitro method to simulate phenolic compound release from the food matrix in the gastrointestinal tract. Eur. Food Res. Technol. 214(2): 155-159.

Glaeser, H., Bujok, K., Schmidt, I., Fromm, M.F., and Mandery, K. (2014). Organic anion transporting polypeptides and organic cation transporter 1 contribute to the cellular uptake of the flavonoid quercetin. Naunyn Schmiedebergs Arch. Pharmacol. 387(9): 883-891.

Goel, G., Kumar, A., Beniwal, V., Raghav, M., Puniya, A.K., and Singh, K. (2011). Degradation of tannic acid and purification and characterization of tannase from Enterococcus faecalis. Int. Biodeterior. Biodegr. 65(7): 1061-1065

González-Barrio, R., Christine, E., and Crozier, A. (2011a). Colonic catabolism of ellagitannins, ellagic acid and raspberry anthocyanins: in vivo and in vitro studies. Drug Metab. Dispos. 39(9): 1680-1688.

González-Barrio, R., Edwards, C.A., and Crozier, A. (2011b). Colonic catabolism of ellagitannins, ellagic acid, and raspberry anthocyanins: in vivo and in vitro studies. Drug Metab. Dispos. 39(9): 1680-1688.

Goto, T., Horita, M., Nagai, H., Nagatomo, A., Nishida, N., Matsuura, Y., and Nagaoka, S. (2012). Tiliroside, a glycosidic flavonoid, inhibits carbohydrate digestion and glucose absorption in the gastrointestina tract. Mol. Nutr. Food Res. 56(3): 435-445.

Gould, G.W., and Holman, G.D. (1993). The glucose transporter family: structure, function and tissue-specific expression. Biochem. J. 295(Pt 2): 329.

Gradolatto, A., Canivenc-Lavier, M.-C., Basly, J.-P., Siess, M.-H., and Teyssier, C. (2004). Metabolism of apigenin by rat liver phase I and phase II enzymes and by isolated perfused rat liver. Drug Metab. Dispos. 32(1): 58-65.

Graf, B.A., Mullen, W., Stewart, C.T., Hartley, R.C., Duthie, G.G., Lean, M.E., Crozier, A., and Edwards, C.A. (2005). Disposition and metabolism of [2-14C] quercetin-4'-glucoside in rats. Drug Metab. Dispos. 33(7): 1036-1043.

Grant, D., and Patel, J. (1969). The non-oxidative decarboxylation ofp-hydroxybenzoic acid, gentisic acid, protocatechuic acid and gallic acid byKlebsiella aerogenes (Aerobacter aerogenes). Antonie van Leeuwenhoek 35(1): 325-343.

Griffiths, L., and Smith, G. (1972). Metabolism of apigenin and related compounds in the rat. Metabolite formation in vivo and by the intestinal microflora in vitro. Biochem. J. 128(4): 901.

Gross, G., Jacobs, D.M., Peters, S., Possemiers, S., van Duynhoven, J., Vaughan, E.E., and Van de Wiele, T. (2010). In vitro bioconversion of polyphenols from black tea and red wine/grape juice by human intestinal microbiota displays strong interindividual variability. J. Agric Food Chem. 58(18): 10236-10246.

Grosser, G., Döring, B., Ugele, B., Geyer, J., Kulling, S.E., and Soukup, S.T. (2015). Transport of the soy isoflavone daidzein and its conjugative metabolites by the carriers SOAT, NTCP, OAT4, and OATP2B1. Arch. Toxicol. 89(12): 2253-2263.

Guengerich, F.P. (2007). Cytochrome p450 and chemical toxicology. Chem. Res. Toxicol. 21(1): 70-83.
Gugler, R., Leschik, M., and Dengler, H. (1975). Disposition of quercetin in man after single oral and intravenous doses. Eur. J. Clin. Pharmacol. 9(2-3): 229-234.

Guidry, A.L., Tibbs, Z.E., Runge-Morris, M., and Falany, C.N. (2017). Expression, purification and characterization of human cytosolic sulfotransferase (SULT) 1C4. Horm. Mol. Biol. Clin. Investig. 29(1): 27-36.

Gutiérrez-Grijalva, E.P., Ambriz-Pére, D.L., Leyva-López, N., Castillo-López, R.I., and Heredia, J.B. (2016). Dietary phenolic compounds, health benefits and bioaccessibility. Arch. Latinoam. Nutr. 66(2): 87-100.

Hackman, R.M., Polagruto, J.A., Zhu, Q.Y., Sun, B., Fujii, H., and Keen, C.L. (2008). Flavanols: digestion, absorption and bioactivity. Phytochem. Rev. 7(1): 195.

Hassaninasab, A., Hashimoto, Y., Tomita-Yokotani, K., and Kobayashi, M. (2011). Discovery of the curcumin metabolic pathway involving a unique enzyme in an intestinal microorganism. Proc. Natl. Acad. Sci. 108(16): 6615-6620.

Hays, W., Jenison, S.A., Yamada, T., Pastuszyn, A., and Glew, R. (1996). Primary structure of the cytosolic beta-glucosidase of guinea pig liver. Biochem. J. 319(Pt 3): 829

He, Z., and Wiegel, J. (1995). Purification and characterization of an oxygen-sensitive reversible 4-hydroxybenzoate decarboxylase from Clostridium hydroxybenzoicum. Eur. J. Biochem. 229(1): 77-82.

Heaney, R.P. (2001). Factors influencing the measurement of bioavailability, taking calcium as a model. J. Nutr. 131(4): 1344S-1348S.

Heider, J., and Fuchs, G. (1997). Anaerobic metabolism of aromatic com pounds. Eur. J. Biochem. 243(3): 577-596.

Heim, K.E., Tagliaferro, A.R., and Bobilya, D.J. (2002). Flavonoid antioxidants: chemistry, metabolism and structure-activity relationships. J. Nutr. Biochem. 13(10): 572-584.

Heinonen, S., Nurmi, T., Liukkonen, K., Poutanen, K., Wähälä, K., Deyama, T., Nishibe, S., and Adlercreutz, H. (2001). In vitro metabolism of plant lignans: new precursors of mammalian lignans enterolactone and enterodiol. J. Agric. Food Chem. 49(7): 3178-3186.

Hemery, Y.M., Anson, N.M., Havenaar, R., Haenen, G.R., Noort, M.W., and Rouau, X. (2010). Dry-fractionation of wheat bran increases the bioaccessibility of phenolic acids in breads made from processed bran fractions. Food Res. Int. 43(5): 1429-1438.

Hidalgo, M., Oruna-Concha, M.J., Kolida, S., Walton, G.E., Kallithraka, S., Spencer, J.P., and de Pascual-Teresa, S. (2012). Metabolism of anthocyanins by human gut microflora and their influence on gut bacterial growth. J. Agric. Food Chem. 60(15): 3882-3890.

Hithamani, G., and Srinivasan, K. (2014). Effect of domestic processing on the polyphenol content and bioaccessibility in finger millet (Eleusine coracana) and pearl millet (Pennisetum glaucum). Food Chem. 164 55-62.

Hodgson, E., and Goldstein, J. (2001). Introduction to biochemical toxicology. Toxicology 2: 2.

Hollman, P.C. (2004). Absorption, bioavailability, and metabolism of flavonoids. Pharm. Biol. 42(sup1): 74-83.

Hollman, P.C., de Vries, J.H., van Leeuwen, S.D., Mengelers, M.J., and Katan, M.B. (1995). Absorption of dietary quercetin glycosides and quercetin in healthy ileostomy volunteers. Am. J. Clin. Nutr. 62(6): 1276-1282.

Hollman, P.C., Van Trijp, J.M., Buysman, M.N., vd Gaag, M.S., Mengelers, M.J., De Vries, J.H., and Katan, M.B. (1997). Relative bioavailability of the antioxidant flavonoid quercetin from various foods in man. FEBS Lett. 418(1-2): 152-156.

Hong, S.S., Seo, K., Lim, S.-C., and Han, H.-K. (2007). Interaction characteristics of flavonoids with human organic anion transporter 1 (hOAT1) and 3 (hOAT3). Pharmacol. Res. 56(6): 468-473.

Hoshino, J., Park, E.-J., Kondratyuk, T.P., Marler, L., Pezzuto, J.M., Van Breemen, R.B., Mo, S., Li, Y., and Cushman, M. (2010). Selective synthesis and biological evaluation of sulfate-conjugated resveratrol metabolites. J. Med. Chem. 53(13): 5033-5043.

Hossain, S.J., Kato, H., Aoshima, H., Yokoyama, T., Yamada, M., and Hara Y. (2002). Polyphenol-induced inhibition of the response of $\mathrm{Na}+/$ glucose cotransporter expressed in Xenopus oocytes. J. Agric. Food Chem. 50(18): 5215-5219.

Hu, J.-L., Nie, S.-P., Min, F.-F., and Xie, M.-Y. (2013). Artificial simulated saliva, gastric and intestinal digestion of polysaccharide from the seeds of Plantago asiatica L. Carbohydr. Polym. 92(2): 1143-1150. 
Hui, Y., Luo, L., Zhang, L., Kurogi, K., Zhou, C., Sakakibara, Y., Suiko, M., and Liu, M.-C. (2015). Sulfation of afimoxifene, endoxifen, raloxifene, and fulvestrant by the human cytosolic sulfotransferases (SULTs): A systematic analysis. J. Pharmacol. Sci. 128(3): 144-149.

Hur, H.-G., and Rafii, F. (2000). Biotransformation of the isoflavonoids biochanin A, formononetin, and glycitein by Eubacterium limosum. FEMS Microbiol Lett. 192(1): 21-25.

Hur, S.J., Lim, B.O., Decker, E.A., and McClements, D.J. (2011). In vitro human digestion models for food applications. Food Chem. 125(1): $1-12$

Ireson, C.R., Jones, D.J., Orr, S., Coughtrie, M.W., Boocock, D.J., Williams, M.L., Farmer, P.B., Steward, W.P., and Gescher, A.J. (2002). Metabolism of the cancer chemopreventive agent curcumin in human and rat intestine. Cancer Epidemiol. Biomarkers Prev. 11(1): 105-111.

Jakoby, W.B. (2012). Enzymatic basis of detoxication, Elsevier, Vol. 1.

Jancova, P., Anzenbacher, P., and Anzenbacherova, E. (2010). Phase II drug metabolizing enzymes. Biomed. Pap. Med. Fac. Univ. Palacky Olomouc Czech Repub. 154(2): 103-116.

Jandhyala, S.M., Talukdar, R., Subramanyam, C., Vuyyuru, H., Sasikala, M., and Reddy, D.N. (2015). Role of the normal gut microbiota. World J. Gastroenterol. 21(29): 8787.

Kadlubar, S., and Kadlubar, F.F. (2010). Enzymatic basis of phase I and phase II drug metabolism Enzyme-and transporter-based drug-drug interactions Springer, 3-25.

Kamiloglu, S., Ozkan, G., Isik, H., Horoz, O., Van Camp, J., and Capanoglu, E. (2017). Black carrot pomace as a source of polyphenols for enhancing the nutritional value of cake: An in vitro digestion study with a standardized static model. LWT-Food Sci. Technol. 77: 475-481.

Kamiloglu, S., Pasli, A.A., Ozcelik, B., Van Camp, J., and Capanoglu, E. (2015). Influence of different processing and storage conditions on in vitro bioaccessibility of polyphenols in black carrot jams and marmalades. Food Chem. 186: 74-82.

Kanazawa, K. (2011). Bioavailability of non-nutrients for preventing lifestyle-related diseases. Trends Food Sci. Technol. 22(12): 655-659.

Kim, D.-H., Yoon, H.-K., Koizumi, M., and Kobashi, K. (1992). Sulfation of phenolic antibiotics by sulfotransferase obtained from a human intestinal bacterium. Chem. Pharm. Bull. 40(4): 1056-1057.

King, C., Rios, G., Assouline, J., and Tephly, T. (1999). Expression of UDPglucuronosyltransferases (UGTs) $2 B 7$ and $1 A 6$ in the human brain and identification of 5-hydroxytryptamine as a substrate. Arch. Biochem. Biophys. 365(1): 156-162.

King, C., Rios, G., Green, M., and Tephly, T. (2000). UDP-glucuronosyltransferases. Curr. Drug Metab. 1(2): 143-161.

Kluge, C., Tschech, A., and Fuchs, G. (1990). Anaerobic metabolism of resorcyclic acids (m-dihydroxybenzoic acids) and resorcinol (1, 3-benzenediol) in a fermenting and in a denitrifying bacterium. Arch. Microbiol. 155(1): 68-74.

Knights, K.M., Rowland, A., and Miners, J.O. (2013). Renal drug metabolism in humans: the potential for drug-endobiotic interactions involving cytochrome P450 (CYP) and UDP-glucuronosyltransferase (UGT). Br. J. Clin. Pharmacol. 76(4): 587-602.

Knockaert, D., Raes, K., Wille, C., Struijs, K., and Van Camp, J. (2012). Metabolism of ferulic acid during growth of Lactobacillus plantarum and Lactobacillus collinoides. J. Sci. Food Agric. 92(11): 2291-2296.

Kobayashi, S., Shinohara, M., Nagai, T., and Konishi, Y. (2013). Transport mechanisms for soy isoflavones and microbial metabolites dihydrogenistein and dihydrodaidzein across monolayers and membranes. Biosci. Biotechnol. Biochem. 77(11): 2210-2217.

Koepsell, H., and Endou, H. (2004). The SLC22 drug transporter family. Pflügers Archiv. 447(5): 666-676.

Konishi, Y., Hitomi, Y., and Yoshioka, E. (2004a). Intestinal absorption of p-coumaric and gallic acids in rats after oral administration. J. Agric. Food Chem. 52(9): 2527-2532.

Konishi, Y., and Kobayashi, S. (2004b). Microbial metabolites of ingested caffeic acid are absorbed by the monocarboxylic acid transporter (MCT) in intestinal Caco-2 cell monolayers. J. Agric. Food Chem. 52(21): 6418-6424.

Konishi, Y., and Kobayashi, S. (2005). Transepithelial transport of rosmarinic acid in intestinal Caco-2 cell monolayers. Biosci. Biotechnol. Biochem. 69(3): 583-591.

Konishi, Y., Kobayashi, S., and Shimizu, M. (2003). Transepithelial transport of p-coumaric acid and gallic acid in Caco-2 cell monolayers. Biosci. Biotechnol. Biochem. 67(11): 2317-2324.

Konishi, Y., Zhao, Z., and Shimizu, M. (2006). Phenolic acids are absorbed from the rat stomach with different absorption rates. J. Agric. Food Chem. 54(20): 7539-7543.

Kroes, I., Lepp, P.W., and Relman, D.A. (1999). Bacterial diversity within the human subgingival crevice. Proc. Natl. Acad. Sci. 96(25): 1454714552.

Kroon, P.A., Faulds, C.B., Ryden, P., Robertson, J.A., and Williamson, G. (1997). Release of covalently bound ferulic acid from fiber in the human colon. J. Agric. Food Chem. 45(3): 661-667.

Krumholz, L., Crawford, R., Hemling, M., and Bryant, M. (1987). Metabolism of gallate and phloroglucinol in Eubacterium oxidoreducens via 3-hydroxy-5-oxohexanoate. J. Bacteriol. 169(5): 1886-1890.

Kullak-Ublick, G.A., Beuers, U., and Paumgartner, G. (2000). Hepatobiliary transport. J. Hepatol. 32: 3-18.

Kurogi, K., Shimohira, T., Kouriki-Nagatomo, H., Zhang, G., Miller, E.R., Sakakibara, Y., Suiko, M., and Liu, M.-C. (2017). Human Cytosolic Sulphotransferase SULT1C3: genomic analysis and functional characterization of splice variant SULT1C3a and SULT1C3d. J. Biochem. 162(6): 403-414.

Labib, S., Erb, A., Kraus, M., Wickert, T., and Richling, E. (2004). The pig caecum model: a suitable tool to study the intestinal metabolism of flavonoids. Mol. Nutr. Food Res. 48(4): 326-332.

Labib, S., Hummel, S., Richling, E., Humpf, H.U., and Schreier, P. (2006). Use of the pig caecum model to mimic the human intestinal metabolism of hispidulin and related compounds. Mol. Nutr. Food Res. 50(1): 78-86.

Lafay, S., and Gil-Izquierdo, A. (2008). Bioavailability of phenolic acids. Phytochem. Rev. 7(2): 301

Lafay, S., Gil-Izquierdo, A., Manach, C., Morand, C., Besson, C., and Scalbert, A. (2006). Chlorogenic acid is absorbed in its intact form in the stomach of rats. J. Nutr. 136(5): 1192-1197.

Lai, R.-H., Miller, M.J., and Jeffery, E. (2010). Glucoraphanin hydrolysis by microbiota in the rat cecum results in sulforaphane absorption. Food Funct. 1(2): 161-166

Lai, Y. (2014). Transporters in Drug Discovery and Development: Detailed Concepts and Best Practice. Woodhead Publishing.

Landete, J.M., Curiel, J.A., Rodríguez, H., de las Rivas, B., and Munoz, R. (2008). Study of the inhibitory activity of phenolic compounds found in olive products and their degradation by Lactobacillus plantarum strains. Food Chem. 107(1): 320-326.

Larsson, K., Cavonius, L., Alminger, M., and Undeland, I. (2012). Oxidation of cod liver oil during gastrointestinal in vitro digestion. J. Agric. Food Chem. 60(30): 7556-7564.

Laurent, C., Besançon, P., and Caporiccio, B. (2007). Flavonoids from a grape seed extract interact with digestive secretions and intestinal cells as assessed in an in vitro digestion/Caco-2 cell culture model. Food Chem. 100(4): 1704-1712.

Lee, J.H., Lee, J.E., Kim, Y., Lee, H., Jun, H.-J., and Lee, S.-J. (2014). Multidrug and toxic compound extrusion protein-1 (MATE1/SLC47A1) is a novel flavonoid transporter. J. Agric. Food Chem. 62(40): 9690-9698.

Ley, R.E., Hamady, M., Lozupone, C., Turnbaugh, P.J., Ramey, R.R., Bircher, J.S., Schlegel, M.L., Tucker, T.A., Schrenzel, M.D., and Knight, R. (2008). Evolution of mammals and their gut microbes. Science 320(5883): 1647-1651

Li, G., Yang, M., Zhou, K., Zhang, L., Tian, L., Lv, S., Jin, Y., Qian, W., Xiong, H., and Lin, R. (2015). Diversity of duodenal and rectal microbiota in biopsy tissues and luminal contents in healthy volunteers. J. Microbiol. Biotechnol. 25(7): 1136-1145.

Li, H., Deng, Z., Liu, R., Loewen, S., and Tsao, R. (2014). Bioaccessibility, in vitro antioxidant activities and in vivo anti-inflammatory activities of a purple tomato (Solanum lycopersicum L.). Food Chem. 159: 353-360.

Li, J., Jia, H., Cai, X., Zhong, H., Feng, Q., Sunagawa, S., Arumugam, M., Kultima, J.R., Prifti, E., and Nielsen, T. (2014). An integrated catalog of reference genes in the human gut microbiome. Nat. Biotechnol. 32(8): 834

Liang, L., Wu, X., Zhao, T., Zhao, J., Li, F., Zou, Y., Mao, G., and Yang, L. (2012). In vitro bioaccessibility and antioxidant activity of anthocyanins from mulberry (Morus atropurpurea Roxb.) following simulated 
gastro-intestinal digestion. Food Res. Int. 46(1): 76-82.

Liu, Z., Saarinen, N.M., and Thompson, L.U. (2006). Sesamin is one of the major precursors of mammalian lignans in sesame seed (Sesamum indicum) as observed in vitro and in rats. J. Nutr. 136(4): 906-912.

Loke, W.M., Proudfoot, J.M., Stewart, S., McKinley, A.J., Needs, P.W., Kroon, P.A., Hodgson, J.M., and Croft, K.D. (2008). Metabolic transformation has a profound effect on anti-inflammatory activity of flavonoids such as quercetin: lack of association between antioxidant and lipoxygenase inhibitory activity. Biochem. Pharmacol. 75(5): 1045-1053.

Lončar, J., Popović, M., Krznar, P., Zaja, R., and Smital, T. (2016). The first characterization of multidrug and toxin extrusion (MATE/SLC47) proteins in zebrafish (Danio rerio). Sci. Rep. 6: 28937.

Lu, X., Jiang, K., Han, L., Zhang, M., Zhou, Y., Ma, Y., Zhou, Y., and Meng, S. (2015). Sulfonation of curcuminoids: Characterization and contribution of individual SULT enzymes. Mol. Nutr. Food Res. 59(4): 634-645.

Lucas-Gonzalez, R., Navarro-Coves, S., Pérez-Álvarez, J.A., FernándezLópez, J., Muñoz, L.A., and Viuda-Martos, M. (2016). Assessment of polyphenolic profile stability and changes in the antioxidant potential of maqui berry (Aristotelia chilensis (Molina) Stuntz) during in vitro gastrointestinal digestion. Ind. Crop Prod. 94: 774-782.

Ludwig, I.A., Paz de Peña, M., Concepción, C., and Alan, C. (2013). Catabolism of coffee chlorogenic acids by human colonic microbiota. Biofactors 39(6): 623-632.

Lupa, B., Lyon, D., Shaw, L.N., Sieprawska-Lupa, M., and Wiegel, J. (2008). Properties of the reversible nonoxidative vanillate/4-hydroxybenzoate decarboxylase from Bacillus subtilis. Can. J. Microbiol. 54(1): $75-81$

Madhujith, T., and Shahidi, F. (2009). Antioxidant potential of barley as affected by alkaline hydrolysis and release of insoluble-bound phenolics. Food Chem. 117(4): 615-620.

Maeda-Yamamoto, M., Tachibana, H., and Monobe, M. (2017). 19 Immune Regulatory Effect of Green Tea. Health Benefits of Green Tea: An Evidence-based Approach 161.

Maestro, A., Terdoslavich, M., Vanzo, A., Kuku, A., Tramer, F., Nicolin, V., Micali, F., Decorti, G., and Passamonti, S. (2009). Expression of bilitranslocase in the vascular endothelium and its function as a flavonoid transporter. Cardiovasc. Res. 85(1): 175-183.

Manach, C., Williamson, G., Morand, C., Scalbert, A., and Rémésy, C. (2005). Bioavailability and bioefficacy of polyphenols in humans. I. Review of 97 bioavailability studies. Am. J. Clin. Nutr. 81(1): 230S242S.

Margaillan, G., Rouleau, M., Fallon, J.K., Caron, P., Villeneuve, L., Turcotte, V., Smith, P.C., Joy, M.S., and Guillemette, C. (2015). Quantitative profiling of human renal UGTs and glucuronidation activity: a comparison of normal and tumoral kidney tissues. Drug Metab. Dispos. 43(4): 611-619.

Marsilio, V., and Lanza, B. (1998). Characterisation of an oleuropein degrading strain of Lactobacillus plantarum. Combined effects of compounds present in olive fermenting brines (phenols, glucose and $\mathrm{NaCl}$ ) on bacterial activity. J. Sci. Food Agric. 76(4): 520-524.

Martin, K.R., and Appel, C.L. (2009). Polyphenols as dietary supplements: a double-edged sword. Nutr. Diet. 2010(Suppl. 2): 1-12.

Martin, P.M., Dun, Y., Mysona, B., Ananth, S., Roon, P., Smith, S.B., and Ganapathy, V. (2007). Expression of the sodium-coupled monocarboxylate transporters SMCT1 (SLC5A8) and SMCT2 (SLC5A12) in retina. Invest. Ophthalmol. Vis. Sci. 48(7): 3356-3363.

Martins, N., Barros, L., and Ferreira, I.C. (2016). In vivo antioxidant activity of phenolic compounds: Facts and gaps. Trends Food Sci. Technol. 48: 1-12.

Matsson, P., and Bergström, C.A. (2015). Computational modeling to predict the functions and impact of drug transporters. In Silico Pharmacol. 3(1): 8.

Matthies, A., Loh, G., Blaut, M., and Braune, A. (2011). Daidzein and Genistein Are Converted to Equol and 5-Hydroxy-Equol by Human Intestinal Slackia isoflavoniconvertens in Gnotobiotic Rats-3. J. Nutr. 142(1): 40-46

McCall, A.L., Van Bueren, A.M., Huang, L., Stenbit, A., Celnik, E., and Charron, M.J. (1997). Forebrain endothelium expresses GLUT4, the insulin-responsive glucose transporter. Brain Res. 744(2): 318-326.

McDougall, G.J., Dobson, P., Smith, P., Blake, A., and Stewart, D. (2005). Assessing potential bioavailability of raspberry anthocyanins using an in vitro digestion system. J. Agric. Food Chem. 53(15): 5896-5904.

Merello, M., Lees, A.J., Webster, R., Bovingdon, M., and Gordin, A. (1994) Effect of entacapone, a peripherally acting catechol-O-methyltransferase inhibitor, on the motor response to acute treatment with levodopa in patients with Parkinson's disease. J. Neurol. Neurosurg. Psychiatry 57(2): 186-189.

Miksits, M., Wlcek, K., Svoboda, M., Kunert, O., Haslinger, E., Thalhammer T., Szekeres, T., and Jäger, W. (2009). Antitumor activity of resveratrol and its sulfated metabolites against human breast cancer cells. Planta Med. 75(11): 1227-1230.

Milbury, P.E. (2009). 19 Transport of Flavonoids into the Brain. Micron. Brain Health 265

Minekus, M., Alminger, M., Alvito, P., Ballance, S., Bohn, T., Bourlieu, C., Carriere, F., Boutrou, R., Corredig, M., and Dupont, D. (2014). A standardised static in vitro digestion method suitable for food-an international consensus. Food Funct. 5(6): 1113-1124.

Monagas, M., Khan, N., Andrés-Lacueva, C., Urpí-Sardá, M., Vázquez-Agell, M., Lamuela-Raventós, R.M., and Estruch, R. (2009). Dihydroxylated phenolic acids derived from microbial metabolism reduce lipopolysaccharide-stimulated cytokine secretion by human peripheral blood mononuclear cells. Br. J. Nutr. 102(2): 201-206.

Monagas, M., Urpi-Sarda, M., Sánchez-Patán, F., Llorach, R., Garrido, I., Gómez-Cordovés, C., Andres-Lacueva, C., and Bartolomé, B. (2010). Insights into the metabolism and microbial biotransformation of dietary flavan-3-ols and the bioactivity of their metabolites. Food Funct. 1(3): 233-253.

Morand, C., Crespy, V., Manach, C., Besson, C., Demigne, C., and Remesy, C. (1998). Plasma metabolites of quercetin and their antioxidant properties. Am. J. Physiol. Regul. Integr. Comp. Physiol. 275(1): R212R219.

Mosele, J.I., Macià, A., and Motilva, M.-J. (2015). Metabolic and microbial modulation of the large intestine ecosystem by non-absorbed diet phenolic compounds: a review. Molecules 20(9): 17429-17468.

Mostaghel, E.A., Zhang, A., and Plymate, S. (2016). UDP-glucuronosyltransferase enzymes in prostate cancer progression: is only androgen catabolism involved? Eur. Urol. 69(4): 610-612.

Nielsen, S., Breinholt, V., Cornett, C., and Dragsted, L. (2000). Biotransformation of the citrus flavone tangeretin in rats. Identification of metabolites with intact flavane nucleus. Food Chem. Toxicol. 38(9): 739-746.

Nielsen, S., Breinholt, V., Justesen, U., Cornett, C., and Dragsted, L. (1998). In vitro biotransformation of flavonoids by rat liver microsomes. Xenobiotica 28(4): 389-401.

Nissinen, E., Tuominen, R., Perhoniemi, V., and Kaakkola, S. (1988). Catechol-O-methyltransferase activity in human and rat small intestine. Life Sci. 42(25): 2609-2614.

O'Callaghan, Y., and O'Brien, N. (2010). Bioaccessibility, cellular uptake and transepithelial transport of $\alpha$-tocopherol and retinol from a range of supplemented foodstuffs assessed using the caco-2 cell model. J. Food Sci. Technol. 45(7): 1436-1442.

O'Leary, K.A., Day, A.J., Needs, P.W., Mellon, F.A., O’Brien, N.M., and Williamson, G. (2003). Metabolism of quercetin-7-and quercetin3-glucuronides by an in vitro hepatic model: the role of human $\beta$-glucuronidase, sulfotransferase, catechol-O-methyltransferase and multi-resistant protein 2 (MRP2) in flavonoid metabolism. Biochem. Pharmacol. 65(3): 479-491.

Oh, W.Y., and Shahidi, F. (2018). Antioxidant activity of resveratrol ester derivatives in food and biological model systems. Food Chem. 261: 267-273.

Oliveira, A., and Pintado, M. (2015). Stability of polyphenols and carotenoids in strawberry and peach yoghurt throughout in vitro gastrointestinal digestion. Food Funct. 6(5): 1611-1619.

Otake, Y., Hsieh, F., and Walle, T. (2002a). Glucuronidation versus oxidation of the flavonoid galangin by human liver microsomes and hepatocytes. Drug Metab. Dispos. 30(5): 576-581.

Otake, Y., and Walle, T. (2002b). Oxidation of the flavonoids galangin and kaempferide by human liver microsomes and CYP1A1, CYP1A2, and CYP2C9. Drug Metab. Dispos. 30(2): 103-105.

Pan, Y., Li, H., Zheng, S., Zhang, B., and Deng, Z.-Y. (2018). Implication of the Significance of Dietary Compatibility: Based on the Antioxidant and Anti-Inflammatory Interactions with Different Ratios of Hydrophilic 
and Lipophilic Antioxidants among Four Daily Agricultural Crops. J. Agric. Food Chem. 66(28): 7461-7474.

Passamonti, S., Terdoslavich, M., Franca, R., Vanzo, A., Tramer, F., Braidot, E., Petrussa, E., and Vianello, A. (2009). Bioavailability of flavonoids: a review of their membrane transport and the function of bilitranslocase in animal and plant organisms. Curr. Drug Metab. 10(4): 369394.

Passamonti, S., Vrhovsek, U., and Mattivi, F. (2002). The interaction of anthocyanins with bilitranslocase. Biochem. Biophys. Res. Commun. 296(3): 631-636.

Passamonti, S., Vrhovsek, U., Vanzo, A., and Mattivi, F. (2003). The stomach as a site for anthocyanins absorption from food 1. FEBS Lett. 544(1-3): 210-213.

Patel, S. (2014). Blueberry as functional food and dietary supplement: The natural way to ensure holistic health. Mediterr. J. Nutrition Metab. 7(2): 133-143.

Pei, Z., Bini, E.J., Yang, L., Zhou, M., Francois, F., and Blaser, M.J. (2004). Bacterial biota in the human distal esophagus. Proc. Natl. Acad. Sci. 101(12): 4250-4255.

Peng, H., Li, W., Li, H., Deng, Z., and Zhang, B. (2017). Extractable and nonextractable bound phenolic compositions and their antioxidant properties in seed coat and cotyledon of black soybean (Glycinemax (L.) merr). J. Funct. Food 32: 296-312.

Piskula, M.K., Yamakoshi, J., and Iwai, Y. (1999). Daidzein and genistein but not their glucosides are absorbed from the rat stomach. FEBS Lett. 447(2-3): 287-291.

Poquet, L., Clifford, M.N., and Williamson, G. (2008). Transport and metabolism of ferulic acid through the colonic epithelium. Drug Metab. Dispos. 36(1): 190-197.

Possemiers, S., Heyerick, A., Robbens, V., De Keukeleire, D., and Verstraete, W. (2005). Activation of proestrogens from hops (Humulus lupulus L.) by intestinal microbiota; conversion of isoxanthohumol into 8-prenylnaringenin. J. Agric. Food Chem. 53(16): 6281-6288.

Proano, M., Camilleri, M., Phillips, S.F., Brown, M.L., and Thomforde, G.M. (1990). Transit of solids through the human colon: regional quantification in the unprepared bowel. Am. J. Physiol. Gastrointest. Liver Physiol. 258(6): G856-G862.

Ramakrishna, B.S. (2013). Role of the gut microbiota in human nutrition and metabolism. J. Gastroenterol. Hepatol. 28: 9-17.

Reboul, E., Richelle, M., Perrot, E., Desmoulins-Malezet, C., Pirisi, V., and Borel, P. (2006). Bioaccessibility of carotenoids and vitamin E from their main dietary sources. J. Agric. Food Chem. 54(23): 8749-8755.

Rechner, A.R., Smith, M.A., Kuhnle, G., Gibson, G.R., Debnam, E.S., Srai, S.K.S., Moore, K.P., and Rice-Evans, C.A. (2004). Colonic metabolism of dietary polyphenols: influence of structure on microbial fermentation products. Free Radical Biol. Med. 36(2): 212-225.

Rhoden, K.J. (2012). SLC5A8 and its role in tumorigenesis. Atlas Genet Cytogenet Oncol Haematol. 16(6): 436-440.

Ricaboni, D., Mailhe, M., Benezech, A., Cadoret, F., Fournier, P.-E., and Raoult, D. (2017). 'Acidaminococcus timonensis' sp. nov. and 'Acidaminococcus massiliensis' sp. nov. isolated from human gut. New Microbes New Infect. 15: 46-48.

Riches, Z., Stanley, E.L., Bloomer, J.C., and Coughtrie, M.W. (2009). Quantitative evaluation of the expression and activity of five major sulfotransferases (SULTS) in human tissues: the SULT "pie". Drug Metab. Dispos. 37(11): 2255-2261.

Rodríguez-Roque, M.J., Rojas-Graü, M.A., Elez-Martínez, P., and MartínBelloso, O. (2013a). Soymilk phenolic compounds, isoflavones and antioxidant activity as affected by in vitro gastrointestinal digestion. Food Chem. 136(1): 206-212.

Rodríguez-Roque, M.J., Rojas-Graü, M.a.A., Elez-Martínez, P., and MartínBelloso, O. (2013b). Changes in vitamin C, phenolic, and carotenoid profiles throughout in vitro gastrointestinal digestion of a blended fruit juice. J. Agric. Food Chem. 61(8): 1859-1867.

Roowi, S., Stalmach, A., Mullen, W., Lean, M.E., Edwards, C.A., and Crozier, A. (2009). Green tea flavan-3-ols: colonic degradation and urinary excretion of catabolites by humans. J. Agric. Food Chem. 58(2): 1296-1304.

Rotches-Ribalta, M., Andres-Lacueva, C., Estruch, R., Escribano, E., and Urpi-Sarda, M. (2012a). Pharmacokinetics of resveratrol metabolic profile in healthy humans after moderate consumption of red wine and grape extract tablets. Pharmacol. Res. 66(5): 375-382.

Rotches-Ribalta, M., Urpi-Sarda, M., Llorach, R., Boto-Ordoñez, M., Jauregui, O., Chiva-Blanch, G., Perez-Garcia, L., Jaeger, W., Guillen, M., and Corella, D. (2012b). Gut and microbial resveratrol metabolite profiling after moderate long-term consumption of red wine versus dealcoholized red wine in humans by an optimized ultra-high-pressure liquid chromatography tandem mass spectrometry method. J. Chromatogr. A 1265: 105-113.

Rowland, A., Miners, J.O., and Mackenzie, P.I. (2013). The UDP-glucuronosyltransferases: their role in drug metabolism and detoxification. Int. J. Biochem. Cell Biol. 45(6): 1121-1132.

Russel, F.G. (2010). Transporters: importance in drug absorption, distribution, and removal. Enzyme- and transporter-based drug-drug interactions: Progress and future challenges Springer, 27-49.

Sabino-Silva, R., Mori, R., David-Silva, A., Okamoto, M., Freitas, H., and Machado, U. (2010). The Na+/glucose cotransporters: from genes to therapy. Braz. J. Med. Biol. Res. 43(11): 1019-1026.

Sakamoto, M., Itoh, T., Tukey, R.H., and Fujiwara, R. (2015). Nicotine regulates the expression of UDP-glucuronosyltransferase (UGT) in humanized UGT1 mouse brain. Drug Metab. Pharmacokinet. 30(4): 269-275.

Sánchez-Patán, F., Cueva, C., Monagas, M., Walton, G.E., Gibson, M., G, R., Quintanilla-López, J.s.E., Lebrón-Aguilar, R., Martin-Alvarez, P., Moreno-Arribas, M.V., and Bartolomé, B.A. (2012). In vitro fermentation of a red wine extract by human gut microbiota: changes in microbial groups and formation of phenolic metabolites. J. Agric. Food Chem. 60(9): 2136-2147.

Sancho, R.A.S., Pavan, V., and Pastore, G.M. (2015). Effect of in vitro digestion on bioactive compounds and antioxidant activity of common bean seed coats. Food Res. Int. 76: 74-78.

Sandusky, G., Mintze, K., Pratt, S., and Dantzig, A. (2002). Expression of multidrug resistance-associated protein 2 (MRP2) in normal human tissues and carcinomas using tissue microarrays. Histopathology 41(1): 65-74.

Saura-Calixto, F., Pérez-Jiménez, J., Touriño, S., Serrano, J., Fuguet, E., Torres, J.L., and Goñi, I. (2010). Proanthocyanidin metabolites associated with dietary fibre from in vitro colonic fermentation and proanthocyanidin metabolites in human plasma. Mol. Nutr. Food Res. 54(7): 939-946.

Scheline, R.R. (1968a). The metabolism of drugs and other organic compounds by the intestinal microflora. Acta Pharmacol. Toxicol. 26(4): 332-342.

Scheline, R.R. (1968b). Studies on the role of the intestinal microflora in the metabolism of coumarin in rats. Acta Pharmacol. Toxicol. 26(4): 325-331.

Schinkel, A.H., and Jonker, J.W. (2003). Mammalian drug efflux transporters of the ATP binding cassette (ABC) family: an overview. Adv. Drug Deliv. Rev. 55(1): 3-29.

Schneider, H., Schwiertz, A., Collins, M.D., and Blaut, M. (1999). Anaerobic transformation of quercetin-3-glucoside by bacteria from the human intestinal tract. Arch. Microbiol. 171(2): 81-91.

Selma, M.V., Espin, J.C., and Tomas-Barberan, F.A. (2009). Interaction between phenolics and gut microbiota: role in human health. J. Agric Food Chem. 57(15): 6485-6501.

Serra, A., Macià, A., Romero, M.-P., Reguant, J., Ortega, N., and Motilva, M.-J. (2012). Metabolic pathways of the colonic metabolism of flavonoids (flavonols, flavones and flavanones) and phenolic acids. Food Chem. 130(2): 383-393.

Serventi, L., Chitchumroonchokchai, C., Riedl, K.M., Kerem, Z., Berhow, M.A., Vodovotz, Y., Schwartz, S.J., and Failla, M.L. (2013). Saponins from soy and chickpea: Stability during beadmaking and in vitro bioaccessibility. J. Agric. Food Chem. 61(27): 6703-6710.

Sesink, A.L., Arts, I.C., De Boer, V.C., Breedveld, P., Schellens, J.H., Hollman, P.C., and Russel, F.G. (2005). Breast cancer resistance protein (Bcrp1/ Abcg2) limits net intestinal uptake of quercetin in rats by facilitating apical efflux of glucuronides. Mol. Pharmacol. 67(6): 1999-2006.

Sesink, A.L., Arts, I.C., Faassen-Peters, M., and Hollman, P.C. (2003). Intestinal uptake of quercetin-3-glucoside in rats involves hydrolysis by lactase phlorizin hydrolase. J. Nutr. 133(3): 773-776.

Sessa, M., Tsao, R., Liu, R., Ferrari, G., and Donsì, F. (2011). Evaluation of the stability and antioxidant activity of nanoencapsulated resveratro 
during in vitro digestion. J. Agric. Food Chem. 59(23): 12352-12360.

Setchell, K.D., Brown, N.M., and Lydeking-Olsen, E. (2002). The clinical importance of the metabolite equol-a clue to the effectiveness of soy and its isoflavones. J. Nutr. 132(12): 3577-3584.

Shahidi, F., and Ambigaipalan, P. (2015). Phenolics and polyphenolics in foods, beverages and spices: Antioxidant activity and health effectsA review. J. Funct. Food 18: 820-897.

Shahidi, F., and Yeo, J. (2016). Insoluble-Bound Phenolics in Food. Molecules 21(9): 1216.

Shi, J., Zheng, H., Yu, J., Zhu, L., Yan, T., Wu, P., Lu, L., Wang, Y., Hu, M., and Liu, Z. (2016). SGLT-1 transport and deglycosylation inside intestinal cells are key steps in the absorption and disposition of calycosin-7-0$\beta$-d-glucoside in rats. Drug Metab. Dispos. 44(3): 283-296.

Shia, C.-S., Tsai, S.-Y., Kuo, S.-C., Hou, Y.-C., and Chao, P.-D.L. (2008). Metabolism and pharmacokinetics of 3, 3', 4', 7-tetrahydroxyflavone (fisetin), 5-hydroxyflavone, and 7-hydroxyflavone and antihemolysis effects of fisetin and its serum metabolites. J. Agric. Food Chem. 57(1): 83-89.

Shimada, T. (2006). Xenobiotic-metabolizing enzymes involved in activation and detoxification of carcinogenic polycyclic aromatic hydrocarbons. Drug Metab. Pharmacokinet. 21(4): 257-276.

Sier, J.H. (2015). Investigation of the impact of MRP3 and MRP4 on the disposition of drugs and conjugates in rat and human University of Surrey, United Kingdom.

Silva, I.D., Rodrigues, A., Gaspar, J., Maia, R., Laires, A., and Rueff, J. (1997). Involvement of rat cytochrome $1 \mathrm{~A} 1$ in the biotransformation of kaempferol to quercetin: relevance to the genotoxicity of kaempferol. Mutagenesis 12(5): 383-390.

Siracusa, L., Kulisic-Bilusic, T., Politeo, O., Krause, I., Dejanovic, B., and Ruberto, G. (2011). Phenolic composition and antioxidant activity of aqueous infusions from Capparis spinosa L. and Crithmum maritimum L. before and after submission to a two-step in vitro digestion model. J. Agric. Food Chem. 59(23): 12453-12459.

Skibola, C.F., and Smith, M.T. (2000). Potential health impacts of excessive flavonoid intake. Free Radical Biol. Med. 29(3-4): 375-383.

Smart, R.C., and Hodgson, E. (2018). Molecular and biochemical toxicology, John Wiley \& Sons.

Srinivasan, V.S. (2001). Bioavailability of nutrients: a practical approach to in vitro demonstration of the availability of nutrients in multivitaminmineral combination products. J. Nutr. 131(4): 1349S-1350S.

Stearns, J.C., Lynch, M.D., Senadheera, D.B., Tenenbaum, H.C., Goldberg, M.B., Cvitkovitch, D.G., Croitoru, K., Moreno-Hagelsieb, G., and Neufeld, J.D. (2011). Bacterial biogeography of the human digestive tract. Sci. Rep. 1: 170.

Sun, Y., Tsao, R., Chen, F., Li, H., Wang, J., Peng, H., Zhang, K., and Deng, Z (2017). The phytochemical composition, metabolites, bioavailability and in vivo antioxidant activity of Tetrastigma hemsleyanum leaves in rats. J. Funct. Food 30: 179-193.

Tagliazucchi, D., Verzelloni, E., Bertolini, D., and Conte, A. (2010). In vitro bio-accessibility and antioxidant activity of grape polyphenols. Food Chem. 120(2): 599-606.

Tamai, I. (2012). Oral drug delivery utilizing intestinal OATP transporters. Adv. Drug Deliv. Rev. 64(6): 508-514.

Tan, S., Calani, L., Bresciani, L., Dall'asta, M., Faccini, A., Augustin, M.A., Gras, S.L., and Del Rio, D. (2015). The degradation of curcuminoids in a human faecal fermentation model. Int. J. Food Sci. Nutr. 66(7) 790-796.

Tanihara, Y., Masuda, S., Sato, T., Katsura, T., Ogawa, O., and Inui, K.-I. (2007). Substrate specificity of MATE1 and MATE2-K, human multidrug and toxin extrusions/H+-organic cation antiporters. Biochem. Pharmacol. 74(2): 359-371.

Tarahovsky, Y.S., Kim, Y.A., Yagolnik, E.A., and Muzafarov, E.N. (2014) Flavonoid-membrane interactions: Involvement of flavonoid-metal complexes in raft signaling. Biochim. Biophys. Acta-Biomembr 1838(5): 1235-1246.

Testa, B., and Waterbeemd, H. (2007). ADME-Tox approaches. Compr. Med. Chem. II: 5.

Teubner, W., Meinl, W., Florian, S., Kretzschmar, M., and Glatt, H. (2007). Identification and localization of soluble sulfotransferases in the human gastrointestinal tract. Biochem. J. 404(2): 207-215.

Timbrell, J.A., and Marrs, T.C. (2009). Biotransformation of xenobiotics.
Gen. Appl. Syst. Toxicol.

Toromanović, J., Kovač-Bešović, E., Šapčanin, A., Tahirović, I., Rimpapa, Z., Kroyer, G., and Sofić, E. (2008). Urinary hippuric acid after ingestion of edible fruits. Bosn. J. Basic Med. Sci. 8(1): 38.

Toydemir, G., Capanoglu, E., Kamiloglu, S., Boyacioglu, D., De Vos, R.C., Hall, R.D., and Beekwilder, J. (2013). Changes in sour cherry (Prunus cerasus L.) antioxidants during nectar processing and in vitro gastrointestinal digestion. J. Funct. Food 5(3): 1402-1413.

Tuohy, K.M., Conterno, L., Gasperotti, M., and Viola, R. (2012). Up-regulating the human intestinal microbiome using whole plant foods, polyphenols, and/or fiber. J. Agric. Food Chem. 60(36): 8776-8782.

Turnbaugh, P.J., Hamady, M., Yatsunenko, T., Cantarel, B.L., Duncan, A., Ley R.E., Sogin, M.L., Jones, W.J., Roe, B.A., and Affourtit, J.P. (2009). A core gut microbiome in obese and lean twins. Nature 457(7228): 480

Tzounis, X., Vulevic, J., Kuhnle, G.G., George, T., Leonczak, J., Gibson, G.R., Kwik-Uribe, C., and Spencer, J.P. (2008). Flavanol monomer-induced changes to the human faecal microflora. Br. J. Nutr. 99(4): 782-792.

Vallejo, F., Gil-Izquierdo, A., Pérez-Vicente, A., and García-Viguera, C. (2004). In vitro gastrointestinal digestion study of broccoli inflorescence phenolic compounds, glucosinolates, and vitamin C. J. Agric. Food Chem. 52(1): 135-138.

van der Woude, H., Boersma, M.G., Vervoort, J., and Rietjens, I.M. (2004). Identification of 14 quercetin phase II mono-and mixed conjugates and their formation by rat and human phase II in vitro model systems. Chem. Res. Toxicol. 17(11): 1520-1530.

Velderrain-Rodríguez, G., Palafox-Carlos, H., Wall-Medrano, A., Ayala-Zavala, J., Chen, C.O., Robles-Sánchez, M., Astiazaran-García, H., Alvarez Parrilla, E., and González-Aguilar, G. (2014). Phenolic compounds: their journey after intake. Food Funct. 5(2): 189-197.

Villegas, R., Gao, Y.-T., Yang, G., Li, H.-L., Elasy, T.A., Zheng, W., and Shu, X.O. (2008). Legume and soy food intake and the incidence of type 2 diabetes in the Shanghai Women's Health Study-. Am. J. Clin. Nutr. 87(1): 162-167.

Volk, C. (2014). OCTs, OATs, and OCTNs: structure and function of the polyspecific organic ion transporters of the SLC22 family. Wiley Interdisciplinary Reviews: Membrane Transport and Signaling 3(1): 1-13.

Vyas, K., Shibata, T., Highet, R., Yeh, H., Thomas, P., Ryan, D., Levin, W., and Jerina, D. (1983). Metabolism of alpha-naphthoflavone and betanaphthoflavone by rat liver microsomes and highly purified reconstituted cytochrome P-450 systems. J. Biol. Chem. 258(9): 5649-5659.

Walgren, R.A., Karnaky, K.J., Lindenmayer, G.E., and Walle, T. (2000a). Efflux of dietary flavonoid quercetin $4{ }^{\prime}-\beta$-glucoside across human intestinal Caco-2 cell monolayers by apical multidrug resistance-associated protein-2. J. Pharmacol. Exp. Ther. 294(3): 830-836.

Walgren, R.A., Lin, J.-T., Kinne, R.K.-H., and Walle, T. (2000b). Cellular uptake of dietary flavonoid quercetin $4^{\prime}$ - $\beta$-glucoside by sodium-dependent glucose transporter SGLT1. J. Pharmacol. Exp. Ther. 294(3) 837-843.

Walgren, R.A., Walle, U.K., and Walle, T. (1998). Transport of quercetin and its glucosides across human intestinal epithelial Caco-2 cells. Biochem. Pharmacol. 55(10): 1721-1727.

Walle, T. (2004). Absorption and metabolism of flavonoids. Free Radical Biol. Med. 36(7): 829-837.

Walle, T., Otake, Y., Brubaker, J., Walle, U., and Halushka, P. (2001). Disposition and metabolism of the flavonoid chrysin in normal volunteers. Br. J. Clin. Pharmacol. 51(2): 143-146.

Walle, U.K., Galijatovic, A., and Walle, T. (1999). Transport of the flavonoid chrysin and its conjugated metabolites by the human intestinal cell line Caco-2. Biochem. Pharmacol. 58(3): 431-438.

Walsh, K.R., Zhang, Y.C., Vodovotz, Y., Schwartz, S.J., and Failla, M.L. (2003) Stability and bioaccessibility of isoflavones from soy bread during in vitro digestion. J. Agric. Food Chem. 51(16): 4603-4609.

Wang, L., and Sweet, D.H. (2013). Renal organic anion transporters (SLC22 family): expression, regulation, roles in toxicity, and impact on injury and disease. AAPS J. 15(1): 53-69.

Wang, M., Ahrné, S., Jeppsson, B., and Molin, G. (2005). Comparison of bacterial diversity along the human intestinal tract by direct cloning and sequencing of $16 \mathrm{~S}$ rRNA genes. FEMS Microbiol. Ecol. 54(2): 219-231.

Wang, S., Amigo-Benavent, M., Mateos, R., Bravo, L., and Sarriá, B. (2017). Effects of in vitro digestion and storage on the phenolic content and 
antioxidant capacity of a red grape pomace. Int. J. Food Sci. Nutr. 68(2): 188-200.

Watanabe, H., Yashiro, T., Tohjo, Y., and Konishi, Y. (2006). Non-involvement of the human monocarboxylic acid transporter 1 (MCT1) in the transport of phenolic acid. Biosci. Biotechnol. Biochem. 70(8): 1928-1933.

Watjen, W., Michels, G., Steffan, B., Niering, P., Chovolou, Y., Kampkotter, A., Tran-Thi, Q.-H., Proksch, P., and Kahl, R. (2005). Low concentrations of flavonoids are protective in rat H4IIE cells whereas high concentrations cause DNA damage and apoptosis. J. Nutr. 135(3): 525-531.

Wenzel, U. (2013). Flavonoids as drugs at the small intestinal level. Curr. Opin. Pharmacol. 13(6): 864-868.

Williams, R.J., Spencer, J.P., and Rice-Evans, C. (2004). Flavonoids: antioxidants or signalling molecules? Free Radical Biol. Med. 36(7): 838-849.

Williamson, G., and Clifford, M.N. (2010). Colonic metabolites of berry polyphenols: the missing link to biological activity? Br. J. Nutr. 104(S3): S48-S66.

Willson, K., and Situ, C. (2018). Systematic Review on Effects of Diet on Gut Microbiota in Relation to Metabolic Syndromes. J. Clin. Nutr. Metab. 2017.

Winter, J., Moore, L., Dowell, V., and Bokkenheuser, V. (1989). C-ring cleavage of flavonoids by human intestinal bacteria. Appl. Environ. Microbiol. 55(5): 1203-1208.

Winter, J., Popoff, M., Grimont, P., and Bokkenheuser, V. (1991). Clostridium orbiscindens sp. nov., a human intestinal bacterium capable of cleaving the flavonoid C-ring. Int. J Syst. Evol. microbiol. 41(3): 355357.

Winter, T.N., Elmquist, W.F., and Fairbanks, C.A. (2010). OCT2 and MATE1 provide bidirectional agmatine transport. Mol. Pharmaceut. 8(1): 133-142.

Wolf, B.W., Bauer, L.L., and Fahey, G.C. (1999). Effects of chemical modification on in vitro rate and extent of food starch digestion: an attempt to discover a slowly digested starch. J. Agric. Food Chem. 47(10): 4178-4183.

Wong, Y.C., Zhang, L., Lin, G., and Zuo, Z. (2009). Structure-activity relationships of the glucuronidation of flavonoids by human glucuronosyltransferases. Expert Opin. Drug Metab. Toxicol. 5(11): 1399-1419.

Wood, I.S., and Trayhurn, P. (2003). Glucose transporters (GLUT and SGLT): expanded families of sugar transport proteins. Br. J. Nutr. 89(1): 3-9.

Woodward, G.M., Needs, P.W., and Kay, C.D. (2011). Anthocyanin-derived phenolic acids form glucuronides following simulated gastrointestinal digestion and microsomal glucuronidation. Mol. Nutr. Food Res. 55(3): 378-386

Wu, B., Kulkarni, K., Basu, S., Zhang, S., and Hu, M. (2011). First-pass metabolism via UDP-glucuronosyltransferase: a barrier to oral bioavailability of phenolics. J. Pharm. Sci. 100(9): 3655-3681.

Xie, L.-H., Akao, T., Hamasaki, K., Deyama, T., and Hattori, M. (2003). Biotransformation of pinoresinol diglucoside to mammalian lignans by human intestinal microflora, and isolation of Enterococcus faecalis strain PDG-1 responsible for the transformation of (+)-pinoresinol to (+)-lariciresinol. Chem. Pharm. Bull. 51(5): 508-515.
Xu, C., Li, C.Y.-T., and Kong, A.-N.T. (2005). Induction of phase I, II and III drug metabolism/transport by xenobiotics. Arch. Pharm. Res. 28(3): 249.

Xu, Z., and Knight, R. (2015). Dietary effects on human gut microbiome diversity. Br. J. Nutr. 113(S1): S1-S5.

Yang, I., Jayaprakasha, G., and Patil, B.S. (2018). In vitro digestion with bile acids enhances the bioaccessibility of kale polyphenols. Food Funct.

Yang, J., Qian, D., Jiang, S., Shang, E.-X., Guo, J., and Duan, J.-A. (2012). Identification of rutin deglycosylated metabolites produced by human intestinal bacteria using UPLC-Q-TOF/MS. J. Chromatogr. B 898: 95-100.

Young, L., and Frazer, A. (1987). The fate of lignin and lignin-derived compounds in anaerobic environments. Geomicrobiol J. 5(3-4): 261-293.

Yu, A.S., Hirayama, B.A., Timbol, G., Liu, J., Diez-Sampedro, A., Kepe, V., Satyamurthy, N., Huang, S.-C., Wright, E.M., and Barrio, J.R. (2012) Regional distribution of SGLT activity in rat brain in vivo. Am. J. Physiol. Cell Physiol. 304(3): C240-C247.

Zaupa, M., Scazzina, F., Dall'Asta, M., Calani, L., Del Rio, D., Bianchi, M.A., Melegari, C., De Albertis, P., Tribuzio, G., and Pellegrini, N. (2014). In vitro bioaccessibility of phenolics and vitamins from durum wheat aleurone fractions. J. Agric. Food Chem. 62(7): 1543-1549.

Zhang, B., Deng, Z., Tang, Y., Chen, P.X., Liu, R., Ramdath, D.D., Liu, Q., Hernandez, M., and Tsao, R. (2017). Bioaccessibility, in vitro antioxidant and anti-inflammatory activities of phenolics in cooked green lentil (Lens culinaris). J. Funct. Food 32: 248-255.

Zhang, Q., Rui, X., Li, W., Chen, X., Jiang, M., and Dong, M. (2016). Antiswarming and-biofilm activities of rose phenolic extract during simulated in vitro gastrointestinal digestion. Food Control 64: 189-195.

Zhang, Z., Peng, X., Li, S., Zhang, N., and Wei, H. (2014). Isolation and identification of quercetin degrading bacteria from human fecal microbes. PloS ONE 9(3): e90531.

Zhao, J., and Dixon, R.A. (2009). MATE transporters facilitate vacuolar uptake of epicatechin 3'-O-glucoside for proanthocyanidin biosynthesis in Medicago truncatula and Arabidopsis. Plant Cell 21(8): 2323-2340.

Zhong, Y., and Shahidi, F. (2012). Lipophilised epigallocatechin gallate (EGCG) derivatives and their antioxidant potential in food and biological systems. Food Chem. 131(1): 22-30.

Zhou, M., Xia, L., and Wang, J. (2007). Metformin transport by a newly cloned proton-stimulated organic cation transporter (plasma membrane monoamine transporter) expressed in human intestine. Drug Metab. Dispos. 35(10): 1956-1962.

Zhu, B.T., Ezell, E.L., and Liehr, J.G. (1994). Catechol-O-methyltransferasecatalyzed rapid O-methylation of mutagenic flavonoids. Metabolic inactivation as a possible reason for their lack of carcinogenicity in vivo. J. Biol. Chem. 269(1): 292-299.

Ziegler, K., Kerimi, A., Poquet, L., and Williamson, G. (2016). Butyric acid increases transepithelial transport of ferulic acid through upregulation of the monocarboxylate transporters SLC16A1 (MCT1) and SLC16A3 (MCT4). Arch. Biochem. Biophys. 599: 3-12.

Zoetendal, E.G., Raes, J., Van Den Bogert, B., Arumugam, M., Booijink, C.C., Troost, F.J., Bork, P., Wels, M., De Vos, W.M., and Kleerebezem, M. (2012). The human small intestinal microbiota is driven by rapid uptake and conversion of simple carbohydrates. ISME J. 6(7): 1415. 